\title{
Overexpression of human alpha-Synuclein leads to dysregulated microbiome/metabolites with ageing in a rat model of Parkinson disease
}

Yogesh Singh ${ }^{1,2 \star}$, Christoph Trautwein ${ }^{3}$, Joan Romani ${ }^{4}$, Madhuri S Salker ${ }^{5}$, Peter H Neckel ${ }^{6}$, Isabel Fraccaroli ${ }^{1}$, Mahkameh Abeditashi ${ }^{1}$, Nils Woerner ${ }^{1}$, Jakob Admard ${ }^{1}, 2$, Achal Dhariwal ${ }^{7}$, Morten S Dueholm ${ }^{8}$, Karl-Herbert Schäfer ${ }^{9}$, Florian Lang ${ }^{10}$, Daniel Otzen ${ }^{11}$, Hilal A Lashuel ${ }^{4}$, Olaf Riess ${ }^{1,2 *}$ and Nicolas Casadei ${ }^{1,2 *}$

${ }^{1}$ Institute of Medical Genetics and Applied Genomics, University of Tübingen, Calwerstaße 7, 72076, Germany

${ }^{2}$ NGS Competence Centre Tübingen (NCCT), University of Tübingen, Calwerstaße 7, 72076, Germany

${ }^{3}$ Werner Siemens Imaging Centre (WSIC), Department of Preclinical Imaging and Radiopharmacy, University of Tübingen, Röntgenweg 13, 72076, Germany

${ }^{4}$ École polytechnique fédérale de Lausanne (EPFL) BMI SV LMNN Station 19, $1015 \mathrm{CH}$ Lausanne, Switzerland

${ }^{5}$ Research Institute of Women's Health, University of Tübingen, Calwerstaße 7/6, 72076, Germany

${ }^{6}$ Institute of Clinical Anatomy and Cell Analysis, University of Tübingen, Österbergstraße 3, 72074, Germany

${ }^{7}$ Institute of Oral Biology, University of Oslo, Sognsvannsveien 10, 0316, Norway

${ }^{8}$ Department of Chemistry and Bioscience, Aalborg University, Fredrik Bajers Vej 7H, 9220 Aalborg, Denmark

${ }^{9}$ Enteric Nervous System working Group, University of Applied Sciences Kaiserslautern, Zweibrücken Campus, Amerikastrasse 1, 66482 Zweibrücken, Germany

${ }^{10}$ Institute of Vegetative Physiology, University of Tübingen, Wilhelmstaße 56, 72074, Germany

${ }^{11}$ Interdisciplinary Naonscience Center (iNANO), Aarhus University, Gustav Wieds Vej 14, 8000, Aarhus, Denmark

\section{Short Title: Microbiome dynamics in PD pathogenesis}

Key words: Gut microbiome, PD, intestinal permeability, a-Synuclein, antibiotics

*Address for Correspondence:

Dr Yogesh Singh or Dr Nicolas Casadei

Institute of Medical Genetics and Applied Genomics, University of Tübingen, Calwerstaße 7, 72076, Germany

Phone: +4970712978264

Fax: +49 70712925355

Email: yogesh.singh@med.uni-tuebingen.de;nicolas.casadei@med.uni-tuebingen.de; olaf.riess@med.uni-tuebingen.de 


\section{Abstract}

Since Braak's hypothesis stating that sporadic Parkinson's disease follows a specific progression of the pathology from the peripheral to the central nervous system and can be monitored by detecting accumulation of the alpha-Synuclein protein. There is growing interest in understanding how the gut (commensal) microbiome can regulate alpha-Synuclein accumulation which can lead to PD. We studied a transgenic rat model overexpressing the human alpha-Synuclein and found that the protein overexpression resulted in gut alphaSynuclein expression and aggregation in the gut neurons with advancing age. A progressive gut microbial composition alteration characterized by the reduction of Firmicutes to Bacteroidetes ratio could be detected in the young transgenic rat model and interestingly this ratio was then increased with aging. This observation was accompanied in older animals by intestinal inflammation, increase gut permeability and a robust alteration in metabolites production characterized by the increase of succinate level in the feces and serum. Manipulation of the gut bacteria by short-term antibiotics treatment revealed a complete loss of short-chain fatty acids (SCFAs) and reduction in succinate levels. Although antibiotics treatment did not change alpha-synuclein expression in the enteric nervous system of the colon, it can reduce alpha-synuclein expression in the olfactory bulb of the transgenic rats. In summary, synchronous with ageing, our data emphasize that the gut microbiome dysbiosis leads to a specific alteration of gut metabolites which are reflected in the serum and can be modulated by the environment. 


\section{Introduction}

The traditional hallmark of Parkinson's disease (PD) is the presence of Lewy bodies (LBs), Lewy neurites (LNs) and the loss of dopaminergic (DA) neurons within the substantia nigra pars compacta $(S N p c)^{1-4}$. LBs and $L N s$ are structure resulting from the misfolding and aggregation of the alpha-Synuclein (a-Syn) protein ${ }^{4}$. Exact mechanisms leading to the aggregation of a-Syn and contributing to its toxicity are still unclear. Notably, the dopaminergic neurons of the SNpc appear particularly vulnerable to the effects of a-Syn aggregates and correlating to the clinical phenotype ${ }^{4}$. Surprisingly, new data has revealed that LBs and LNs are also found in the periphery including in nerves of the gastrointestinal $\operatorname{tract}(\mathrm{GIT})^{5}$.

Although PD is classically classified as a disease of the central nervous system, but numerous observations suggest that PD is not a single disease ${ }^{6}$ and that in specific form of $P D$ is originating from peripheral tissues such as the gut epithelial cells called enteroendocrine cells (EECs), neurons of the GIT and olfactory system ${ }^{2,7-9}$. It is suggested that amyloidogenic a-Syn seeds may transfer from neuron to neuron as a result of retrograde transport causing accumulation of a-Syn in the bowel ${ }^{2,10-13}$. At the early stages of the disease, PD patients frequently exhibit non-motor symptoms such as olfactory malfunction, constipation and depression ${ }^{14}$. GIT dysfunction (in particular constipation) is observed in approximately $80 \%$ PD patients (sporadic and familial forms) before the occurrence of motor symptoms ${ }^{14,15}$. Recent patient study indicating two subtypes of PD, (1) brain-to-gut and (2) gut-to-brain with respect to a-Syn pathology, thus, clarifies the issue with different potentially conflicting earlier report in the PD field ${ }^{16}$.

Several studies have highlighted an association of gut microbiome dysbiosis in PD patients compared with healthy controls ${ }^{1,17,18}$. Whilst, individual bacterial composition varied from different PD-cohorts, only a few bacterial genera appeared to be consistent among different studies (e.g. Bifidobacterium, Akkermansia and Lactobacillus) ${ }^{19,20}$. Some were conflicting, in particular the Lactobacillus genus ${ }^{21}$ with some findings suggesting higher abundance ${ }^{17,22-26}$ whilst others suggested a lower abundance of Lactobacillus ${ }^{18,27,28}$. However, the major limitation of these studies is that the (PD patient's) microbiome was quantified when the disease was already established. Further, it is not also known whether these changes in gut microbiome described play a causative role in the pathogenesis or are merely a consequence of disease ${ }^{5}$. Studying the microbiome before disease onset in humans is not possible due to unavailability of any known pre-clinical markers. Thus, rodent models are critical in understanding the physiological role of the gut microbiome on the disease development ${ }^{29}$.

In this context, the presence of synucleinopathy in the autonomic nervous system, accumulation of a-Syn in the bowel as well as altered intestinal microbiota correlated to motor symptoms pointing to a potential origin of synucleinopathy in the gut ${ }^{30}$. Gut bacteria can control the differentiation and function of immune cells in the intestine, periphery and in the brain ${ }^{31-33}$. A murine model that overexpressed the human a-Syn under the thy 1 promoter (Thy1-a-Syn) suggested that gut microbiome transplants from PD patients into these mice induced parkinsonian-like motor dysfunction and that the microbiome was necessary to induce $a$-Syn pathology, neuroinflammation and motor defects ${ }^{29}$. Furthermore, using germfree (GF) environmental conditions, this study showed that the gut microbiome was able to produce short-chain fatty acids (SCFAs) which were upregulated in the Thy1-a-Syn mice ${ }^{29}$. These SCFAs, especially butyrate, protect against intestinal hyperpermeability and decrease 
inflammation through modulation of microglial activation ${ }^{34}$. Additionally, SCFA-producing bacteria are less abundant in PD patients ${ }^{35}$. However, the exact role of SCFAs in neurodegeneration required further investigation due to conflicting results in murine and PD patient. Interestingly, antibiotic treatment in Thy1- $\alpha$-Syn mice decreased the fine and gross motor function as well as gut motility defects highlighting that microbial signals modulate the a-Syn-dependent pathology ${ }^{29}$, thus providing a pivotal link between the gut microbiome and PD pathology. This finding suggests a potential influence of genetics on gut-microbiome interactions and synucleinopathies ${ }^{29}$. However, how a specific gut bacterial genera/species interacts with its host genetics with progressive ageing leading to PD like disease progression remains unknown.

Therefore, we hypothesized that a-Syn overexpression could be involved in modulating the gut microbiome composition and metabolite production which results in the induction of aSyn misfolding and aggregation in enteric neurons. Accordingly, studying the microbiome dynamics, GIT physiology and function could lead to vital new insights into PD pathology. Given the broad pathophysiological effects of PD, we have used a previously described rat model overexpressing full-length non-mutated human a-Syn which includes the entire SNCA sequence with upstream regulatory promoter sequences and a flanking downstream region in a BAC construct ${ }^{36}$. Further, the rat BAC-SNCA transgenic PD (referred to as TG) model was shown to present a strong overexpression of $\alpha$-Syn in the brain and to also reproduce the formation of pathological form of a-Syn. These TG rats develop early changes in noveltyseeking, avoidance, locomotor decline and an early smell deficit before the progressive motor deficit ${ }^{36}$. The observed pathological changes were linked with severe loss of structural dopaminergic integrity ${ }^{36,37}$. Moreover, these TG rats also showed bidirectional, trans-synaptic parasympathetic and sympathetic propagation of $a-S y n^{38}$. This TG rat model holds close resemblance to the human PD disease progression, therefore, this model appeared to be ideal for testing the hypothesis that $\alpha$-Syn expression regulates the gut bacterial abundance and pathophysiology of synucleinopathies.

Herein, we studied longitudinally the gut microbiome dynamics with ageing and report that with ageing, overexpression of human a-Syn changes the gut microbiota composition, metabolites and inflammation. Together, these results suggest that the humanized-rat model supports the notion that the gut microbiome could shape the progression of PD pathogenesis.

\section{Results}

\section{Microbiome dynamics in the $\alpha$-Syn TG rats}

To determine the effect of human a-Syn overexpression on the gut microbiome's composition and diversity of the gut microbiome, we analysed faecal pellets from the colon of the wildtype (WT) and TG rats by $16 \mathrm{~S}$ ribosomal RNA (16S rRNA) gene amplicon sequencing. We used homozygous TG and control WT littermate rats, which were obtained from heterozygous mothers (Fig. 1a; described in Materials and Methods section) to negate any maternal (genetic) effects on microbiome analysis. The total numbers of reads between both WT and TG rat samples for each respective age group was shown for our sequencing depth (Suppl. Fig. 1a). Alpha diversity captures both the organismal richness of a sample and the evenness of the organisms' abundance distribution ${ }^{39,40}$. We estimated the population diversity of the microbial community (alpha-diversity) by Chao 1 and Shannon-Weaver index with MicrobiomeAnalyst tool ${ }^{41}$ and MEGAN-CE software ${ }^{42}$ at phylum level. The alpha 
diversity Shannon-Weaver index in TG rats tended to be lower at each time point at phylum level, a trend, however, not reaching significance (Suppl. Fig. 1b). Most of the bacteria belonged to the Firmicutes and Bacteroidetes phyla and, both are typically the dominant phyla in the gut microbiome in humans and animals (Fig. 1b). At each age group, only a few bacterial phyla were significantly altered (WT vs TG comparisons), but most significant changes were observed during $1 \mathrm{M}$ (Proteobacteria; $\mathrm{p}=0.04$ ), $2 \mathrm{M}$ (Aquificae; $\mathrm{p}=0.007$ ) $2.5 \mathrm{M}$ (Firmicutes; $p=0.02$, Bacteroidetes; $p=0.005$, Actinobacteria; $p=0.04$ and Cyanobacteria; $p=0.03$ ), $6 \mathrm{M}$ (Actinobacteria; $p=0.02$, Verrucomicrobia; $p=0.01$ and Chloroflexi; $p=0.01$ ) and $>12 \mathrm{M}(12-14 \mathrm{M})$ (Bacteroidetes; $p=0.02$ ) of age in TG rats (Fig. 1b and Suppl. Fig. 1c). Further, with ageing the Bacteroidetes phylum was significantly reduced in TG rats (Fig. 1b and Suppl. Fig. 1c). The Proteobacteria phylum abundance was increased in $>12 \mathrm{M}$ TG rats, however, it did not reach a significant level (Fig. 1b). Further, the Firmicutes and Bacteroidetes (F/B) ratio was calculated for the respective age group in WT and TG rats. The 2.5M age group had a significant reduction in $\mathrm{F} / \mathrm{B}$ ratio in $\mathrm{TG}$ group (Fig. 1C). In sharp contrast, this ratio was significantly increased at $>12 \mathrm{M}$ old $\mathrm{TG}$ group (Fig. 1c). Further, we performed two-way analysis of variance analysis (Two-way ANOVA) to find a correlation between change in the F/B ratio affected by age and bacteria. Our analysis suggested that with age there is a change in F/B ratio but both factors independently are no correlated (Fig. 1c). Additionally, we analysed the alpha diversities (Shannon-Weaver index and Chao 1) (Suppl. Fig. 1d) and the bacterial composition at the genera level (Fig. 1d). Surprisingly, even at an early age (2.5M), we were able to detect bacterial dysbiosis in TG rats compared to WT control rats and observed that ageing accelerates bacterial dysbiosis dynamics significantly (Fig. 1d and Suppl. Fig. 1e and Suppl. Fig. 2). The most interesting correlation was between Lactobacillus and Alistipes. With ageing TG rats had decreased \% abundance of Lactobacillus whereas \% abundance of Alistipes genus was significantly increased (Fig. 1e, f). Two-way ANOVA suggested that in WT and TG rats, with age there is change in the dynamics of Alistipes and Lactobacillus (Fig. 1e \& f). Thus, overall, bacterial composition at genera level was significantly different between WT and TG rat at any given age group (Suppl. Fig. 2).

Several patients and murine model studies have suggested that Lactobacillus is abundantly present in relation to $\mathrm{PD}^{17,23,24,43}$. In contrast, other studies including ours suggested that Lactobacillus is decreased in PD models as well as in PD patients ${ }^{18,28,44}$. To understand the discrepancies in the Lactobacillus abundance with respect to a-Syn overexpression, we performed a mother to children microbiome follow up study. Microbiome composition analyses suggested that at $2 \mathrm{M}$ of age, WT and TG rats have a different pattern of Lactobacillus $s p$. in their feces (Suppl. Fig. 3a, b, \& c). There was no detectable effect of the cages on the microbiome composition (Suppl. Fig. 3a).

204 Different environments as found in different animal facilities could change the gut 205 microbiome. Therefore, both WT and TG rats were also kept in two different animal facilities 206 (Facility I and Facility II) and tested whether bacterial composition was different or similar. 207 We found that two different animal facilities did have a similar bacterial composition including 208 Lactobacillus abundance (Suppl. Fig. 4a \& b). However, Lactobacillus sp. abundance varied 209 in TG rats, although the trend was less Lactobacillus compared with WT (Suppl. Fig 4b).

210 Next, we investigated whether keeping both genotype rats in the same cage, could change 211 the gut microbiome of older TG rats ('cage transfer transplant') or whether 'genetic factors' 212 were still predominant in determining the microbiome. Thus, we performed a pivotal 213 experiment to answer this conjecture. Firstly, WT and TG (4 females only) rats were kept 
214 separately for $>12 \mathrm{M}$ and at the end of the experimental period faecal pellets were collected 215 (24 hours prior) (Suppl. Fig. 5a). Subsequently, the groups were then divided (2 WT and 2 $216 \mathrm{TG})$ and kept together in fresh cage for one week and faecal pellets collected at the 217 beginning (day 0 ) and end of the experiment (day 7). We found that Lactobacillus remained reduced whereas Alistipes continued to be elevated (Suppl. Fig. 5e \& f). The ratio of Lactobacillus and Alistipes was not changed dramatically (before and after) due to the cage transfer experiment (Suppl. Fig. 5). Thus, in summary, a-Syn overexpression could have a key role in governing the expression of different bacterial genera including Lactobacillus.

Metagenomic bacterial functional analysis in older TG rats

223

224

225

226

227

228

229

230

231

232

233

234

235

236

237

238

239

240

241

The microbial dysbiosis with ageing in TG rats (>12M) was observed by $16 \mathrm{~S}$ rRNA gene amplicons sequencing. However, this method is limited as it relies on the amplification of a single gene using PCR-based of confined regions of the bacterial genome only. To increase the sensitivity of the microbiome sequencing, we performed shotgun sequencing which also allow to sequence the whole bacterial genome and also provides critical metabolic function interaction of the bacterial community. The shotgun sequencing depth and taxonomic rarefaction was shown in Suppl. Fig. 6 for both WT and TG rats. Again, the F/B ratio was significantly higher in TG compared with control WT littermates at phylum level (Fig. 2a, b). The alpha diversity was significantly reduced in TG rats (Fig. 2). Furthermore, based on genera taxonomic unit, we calculated the percentage (\%) composition of the gut bacteria in WT and TG rats and found that Lactobacillus was reduced in $>12 \mathrm{M} \mathrm{TG}$ rats compared with WT, whereas Alistipes was significantly increased in the TG rats (Fig. 2c, d, e \& Suppl. Fig. 6 ). Alpha and beta diversities represent the similarity (or difference) in organismal composition within and in between the samples. Alpha diversity was measured using the Shannon-Weaver index revealing that alpha-diversity was significantly higher in WT compared with TG rats (Fig. 2f). Next, using the principal component analysis (Bray-Curtis method) to investigate beta diversity, we found that the gut microbiome at the genus level from WT and TG rats cluster separately (Fig. 2g). Overall, our data indicate that a-Syn overexpression induces the gut microbiome dysbiosis in ageing TG rats.

\section{$T G$ rats have a dysregulated metabolite production}

To further understand change in the bacterial composition could also lead to different metabolites production, we performed proton nuclear magnetic resonance spectroscopy $\left({ }^{1} \mathrm{H}\right.$ NMR)-based metabolomics from faecal and serum samples of younger (3M) and older (>12M) WT and TG rats (Fig. 3a). In total, we were able to quantify 31 different metabolites from feces and 38 metabolites from serum (Suppl. Fig. 7). Multivariate statistics using Partial Least Squares Discriminant Analysis (PLS-DA) was employed to identify differences between WT and TG rats for each age group (3M and $>12 \mathrm{M}$ ) for the feces (Suppl. Fig. 7a, b) and serum samples (Suppl. Fig. 7c, d). At $>12 \mathrm{M}$ of age, there was a clear separation between WT and TG rat samples (Suppl. Fig. 7b, d), however this gap was less discrete for $3 \mathrm{M}$ age group samples (Suppl. Fig. 7a, c). When comparing variable importance in projection (VIP) scores of the PLS-DA feces analysis, we identified the most important metabolites high levels of succinate in 3 M TG rats whilst high levels of glutamate were discerned in $>12 \mathrm{M}$ WT rats (Suppl. Fig. 7a, b). In serum, 3M WT rats showed elevated glucose levels, whilst $>12 \mathrm{M} \mathrm{TG}$ rats showed high lactate and succinate levels (Suppl. Fig. 7c, d).

To support the findings of multivariate statistics we applied a stringent univariate volcano plot analysis (combination of fold change and t-test). Hereby, we compared the metabolites levels 
based on genotype at each age group (3M and $>12 \mathrm{M})$ when comparisons were made for TG versus WT (TG/WT) (Fig. 3a, b) in the feces and serum respectively. Further, we then compared the levels of the metabolites for each genotype with age ( $3 \mathrm{M}$ to $>12 \mathrm{M}$ ) for WT (Suppl. Fig. 8a) and TG (Fig. 3c). We used FC > $1.2(\log 2$ FC) and p-value $<0.05(-\log 10$ ( $p$ value)) by default for all the metabolites and plotted against both values as volcano plots (Fig. 4 \& Suppl. Fig. 8). At 3M of age, feces genotype comparison TG/WT suggested that phenylacetate was significantly upregulated whereas at $>12 \mathrm{M}$ of age (TG/WT) a divergent set of metabolites such as 4-Hydroxyphenylacetate and lactate were upregulated while glutamate was significantly downregulated (Fig. 3a). We identified the most significant changes in the serum samples from $>12 \mathrm{M}$ old $\mathrm{TG}$ and $\mathrm{WT}$ rats. We observed very high levels of lactate and succinate in TG compared to WT (Fig. 3b). Interestingly, when we explored serum metabolites, we found that glutamate was significantly downregulated at younger age $(3 \mathrm{M})$. In contrast, with ageing succinate, glutamate and lactate were significantly upregulated in older ( $>12 \mathrm{M}$ ) TG rats compared to WT (TG/WT comparisons at a given age) (Fig. 3b). Further, we performed the ageing comparison for each genotype either WT or TG and found that serum succinate in TG rats was significantly upregulated (Fig. 3c; left). Succinate correlation coefficient analysis suggested that tryptophan and tyrosine were negatively correlated with succinate while other SCFAs were positively correlated (Fig. 3c; right). In WT serum succinate tended to decline with ageing, a trend, however, not reaching statistical significance (Suppl. Fig. 8a). Whilst observing the ageing effect for feces, the correlation analysis with succinate suggested tyrosine is negatively correlated, whilst 4hydroxyphenylacetate was positively correlated in TG rats (Suppl. Fig. 8b). Overall, it appears that dysregulated levels of succinate, lactate and tyrosine could be linked with PD progression.

Presence of synucleinopathy in the enteric nervous system (ENS) of TG rats

Using antibodies specific for human a-Syn, we detected using immunohistochemistry specific staining in the colon of TG rats and found presence of human a-Syn expression in the gut of TG rats whereas we did not detect any human a-Syn expression in WT rats (Fig. 4a). Human a-Syn staining was mostly found in the ENS of the colon and also in the myenteric plexus of $\mathrm{TG}$ rats. Presence of the transgenic human a-Syn protein was also validated by immunoblotting (Suppl. Fig. 9a). Expression of the human a-Syn expression was also detected in the myenteric plexus and in the colon of TG rats using the whole-mount immunostainings of the Tunica muscularis (Supp. Fig. 9b).

To investigate the presence of a pathological forms of a-Syn and an accumulation of a-Syn in TG rats, we first performed the histological analysis of the colon. We could detect reproducibly the presence of phosphorylation of human a-Syn at serine 129 position which 295 increased with ageing (Fig. 4b). Presence of synucleinopathy was investigated using trypsin digestion to identify the insoluble forms of a-Syn. Colon sections of WT rats presented a-Syn staining with endogenous rat antibody, however, after trypsin treatment for 2 hours, this staining was lost in the myenteric plexus (Suppl. Fig. 10). Yet, in sharp contrast to WT rats, TG rats exhibited positive staining for total human a-Syn after trypsin digestion even in young 300 rats suggesting that aggregation of a-Syn already occurs at two months of age (Suppl. Fig. 10). Total accumulation of $a-S y n$ is increased with age in TG rats (2M) and reached a plateau at the age of $4 \mathrm{M}$ (Fig. 4c). At later stages (>12M), a-Syn still tended to increase slightly, but no significant change to $4 \mathrm{M}$ was observed (Fig. 4c). Together, our data suggest that human a-Syn accumulation occurs in the colon of young TG rats. 
It was previously described that in the brain samples of TG rats there are several small sized fragments of $a-S y n^{36}$. To test this, we performed immunoblotting of human a-Syn from colon of $T G$ rats and identified several small-sized fragments of a-Syn in different age groups (5M, $>12 \mathrm{M}$ ) (Suppl. Fig. 11a). We found 3 major truncated fragments of human a-Syn protein which we characterized further using specific a-Syn antibodies targeted at $\mathrm{N}$ - or C-terminal epitopes (Suppl. Fig 11c). Truncated fragments did not arise from the boiling of the proteins as samples prepared in the presence of DSP (a cross-linker) led to the same results (Supp. Fig. 11b). Interestingly, a-Syn exhibited two N-terminal truncation fragments and one Cterminal truncation fragment (Suppl. Fig 11c). Thus, a-Syn expression in the colon revealed in addition to brain pathology that intestinal tissue is valuable in assessing PD progression in TG rats.

\section{Physiological functions of the TG rats dysregulated with ageing}

The colon is a significant site of water and salt absorption and, during this process it desiccates the feces. The dysregulation in the gut microbiome and metabolites could influence the intestinal permeability. Therefore, we used an Ussing chamber to evaluate the function of intestinal permeability and $\mathrm{Na}^{+}$uptake. To do so, we measured the transepithelial $\mathrm{Na}^{+}$current $(\mathrm{ENaC})$ in the colon epithelium. We measured the $\mathrm{ENaC}$ current in young (2M) and older (12M) rats of both the genotypes and found that $2 \mathrm{M}$ and $12 \mathrm{M}$ TG rats had a lower ENac current compared with WT rats respectively (Fig. 5a, b, c). This could suggest that TG rats may exhibit a dysregulation of the $\mathrm{Na}^{+}$absorption in the colon. Further, we found that the transepithelial resistance tended to decrease with ageing, however no significant change was observed between WT and TG at any age group (Fig. 5c). Interestingly, the feces' water content showed no difference between 12M WT and TG rats (data not shown). Hypoxanthine is negatively correlated with intestinal inflammation and permeability in inflammatory bowel disease ${ }^{45}$. We found that hypoxanthine is significantly increased in $14 \mathrm{M}$ WT compared with $3 \mathrm{M}$ WT rats but not in TG rats (between $3 \mathrm{M}$ and $>12 \mathrm{M}$ ). Hypoxanthine was not significant between TG and WT rats (Fig. $5 \mathrm{~d}$ ). Nonetheless, gut dysbiotic metabolites could affect the $\mathrm{Na}^{+}$uptake or intestinal permeability in TG rats.

\section{Increased local and systemic inflammation in TG rats}

PD patients have a higher abundance of inflammatory proteins in the feces, possibly be due to dysbiotic changes in the gut microbiome ${ }^{46-48}$. Thus, we measured the faecal inflammatory proteins in TG rats. Faecal calprotectin level was significantly increased in older (8M) TG rats compared with WT, however no change occurred at an earlier age (3M) (Fig. 6a). To understand where the sources of these increased inflammatory proteins in the feces are, we extended our evaluation on the gene expression in the host intestine. Thus, we performed RNA-sequencing (RNA-seq) from the mucosa and submucosal tissues (defined as MS layer) from young (3M) and older (>12M) TG and control WT rats. Our RNA-seq experiments suggested that an enrichment of dysregulated genes involved in inflammation was detected with ageing in TG rats (Fig. 6b-e and Suppl. Fig. 12,13). Further, Ingenuity pathway analysis (IPA) suggested that several inflammatory pathways including innate and adaptive immunity were upregulated in $>12 \mathrm{M} \mathrm{TG}$ rats compared with $3 \mathrm{M} \mathrm{TG}$ rats (Fig. 6b). Most interestingly, PD-1, PD-L1 cancer immunotherapy pathways, antioxidant action of vitamin C, RhoGDI signalling, Apelin cardiac fibroblast signalling pathway and LXR/RXR activation pathways were downregulated significantly in older TG (>12M) compared with $3 M$ TG rats (Fig. 6b, c). More specifically, with ageing NFAT regulation of the immune response, cardiac hypertrophy signalling, neuroinflammation signalling pathway, G-beta gamma signalling, ephrin receptor 
signalling and opioid signalling pathway were upregulated with ageing in $>12 \mathrm{M} \mathrm{TG}$ rats compared to $3 \mathrm{M}$ TG rats (Fig. 6b-e). Additionally, whole genome wide analysis suggested that programmed cell death pathway genes were decreased, whilst immune system pathway genes were increased in old TG rats compared to young TG rats (Fig. 6b \& Suppl. Fig. 13). Therefore, we tested the adaptive immune cells of younger $(3 \mathrm{M})$ and older $(>12 \mathrm{M}) \mathrm{TG}$ rats with their respective age matched WT controls. We found that ageing led to a significant increase in inflammatory IFN- $\gamma$ cytokines from CD4 ${ }^{+} \mathrm{T}$ cells and also increased $\mathrm{CD} 4^{+} \mathrm{T}$ cells (Fig. 6f), whereas no significant difference was found at $3 \mathrm{M}$ age in between WT and TG (data not shown). Overall, data are suggesting that ageing TG rats have an activated inflammatory environment compared with younger TG rats.

Antibiotics treatment affects the gut bacterial diversity, metabolites production and gene expression in the gut

To understand whether reducing the gut bacteria load could lead to changes in the inflammatory environment or a-Syn expression, we treated the WT and TG rats at 2.5M of age with broad-spectrum antibiotics for two weeks. Antibiotic treatment led to a reduction of live bacterial load and further reduced the amount of the total DNA obtained from an equal weight of the stool samples (Fig. 7a, b). The 16S rRNA amplicon sequencing suggested a tendency to reduce the F/B ratio and bacterial alpha diversity (phylum level) after antibiotics treatment for WT and TG rats respectively. However, no significant difference was observed (Fig. 7c-e). Beta diversity (PCoA of taxonomy using Bray-Curtis at phylum level) data suggested that antibiotics treated rats clustered into different groups compared with untreated rats (Fig. 7f).

We isolated the Tunica muscularis containing the myenteric plexus and found by immunoblotting that antibiotic treatment did not affect the total a-Syn expression in the ENS. However, there was a tendency for reduced a-Syn expression in the brain olfactory bulb region but not reaching to a significant level. (Fig. 7g). Wholemount staining of the Tunica muscularis of TG rats from antibiotic treatment and control groups revealed that the expression of a-Syn was present in the myenteric plexus as well as in nerve fibers in the circular and longitudinal muscle layers and that there was no change in expression in response to the antibiotic treatment (Fig. $7 \mathrm{~h}$ ).

Moreover, we performed the RNA-seq analysis of the colon MS layer, we found that several genes were differentially regulated after antibiotics treatment for WT and TG rats respectively (Fig. 7i). IPA analysis suggested that the antibiotics treatment affected 478 genes in TG rats and 521 genes in WT rats (normalised for both the genotypes independently TG-Ab/TG-C and WT-Ab/WT-C). Out of these genes 165 were common in WT and TG. These changes could be caused either by the reduced gut microbiome load or the direct effects of the applied antibiotics (Suppl. Fig. 14). Interestingly, Dnase1 and Trpv1 were two of the genes that were upregulated after antibiotic treatment in TG rats. Genes that were upregulated in both WT and TG rats after antibiotic treatments included Hdac-5, CD86 and Pink1, whereas RoRc, Spink4, Hsp90b1 were downregulated (Suppl. Fig. 14).

Furthermore, we performed ${ }^{1} \mathrm{H}-\mathrm{NMR}$ metabolomics of antibiotic treated rats and identified 45 faecal and 38 serum metabolites. We found that antibiotic treatment was followed by profound effect on the faecal SCFAs, amino acids (e.g. tyrosine, tryptophan, lysine was reduced in WT rats after antibiotics, whereas no change was observed in TG rats) and sugars (Suppl. Fig 15a \& b) while for serum only slight changes could be observed (Suppl. 
Fig. 15c \& d). Most strikingly, multivariate VIP scores analysis of serum samples (Suppl. Fig $14 \mathrm{e}$ ) identified most strikingly reduced succinate levels (Suppl. Fig. 15f) alongside decreased occurrence of the ketone body 3-Hydroxybutyrate (Suppl. Fig. $15 \mathrm{~g}$ ) in both WT and TG rats after antibiotics treatment. Tyrosine was significantly increased with ageing in TG rat feces after antibiotic treatment, while Tryptophan also had a similar trend but not reaching statistical significance (Fig. 7j). Succinate was totally absent after antibiotic treatment in the feces, thus, suggesting the potential role of succinate production by bacterial metabolism (Fig, 7j). These data signify that microbiome manipulation affects the bacterial load, gene expression and metabolite production.

\section{Discussion}

Recent emerging reports have investigated gut microbiome dysregulation and its role in the pathogenesis of $P D^{1,17,18,29,49}$. Most of the studies propose that PD patients undergo changes in the relative quantity of particular types of bacterial genera and, due to these changes in bacterial load, the metabolism of PD patients is affected ${ }^{18,50}$. Altered bacterial genera/species and their changes to metabolite production may also inadvertently modulate the immune response ultimately, leading to inflammation in various tissues and cellular components of the gut including enteric neurons, glial cells, and immune cells ${ }^{31}$. However, the data from PD patients are always limited in numbers and often have a high variance in terms of age differences, body mass index, exposure to antibiotics, diet, ethnicity etc. To study and understand how the gut microbiome is involved in PD pathology, the above-mentioned factors need to be controlled to have a clear understanding. Moreover, it would be critical to identify patients in the pre-clinical stage who are not experiencing any motor and non-motor symptoms. To address these issues, rodent models (mouse or rats) are useful for identifying and performing functional and mechanistic research on host-microbiome interactions as they allow manipulation of genome, environment and gut microbiome composition ${ }^{51}$. In this study, using the TG PD rat model, we observed that overexpression of a-Syn modifies the gut microbiome, metabolite production and induces inflammation.

Current findings have demonstrated that the intestinal microbiota interacts with the autonomic and central nervous system via diverse pathways, including the ENS and Vagal nerve $^{31}$. Studies in PD patients have described that microbial composition in feces and mucosa were significantly different at multiple levels ${ }^{1,17,49}$. The diversity of faecal bacterial communities was not largely different between PD and healthy control subjects ${ }^{43}$. Significant differences in alpha-diversity between PD and healthy control groups were primarily observed at the phylum level and PD disease duration correlated with the majority of taxa, e.g., Bacteroidetes and Proteobacteria being positively correlated and Firmicutes negatively correlated ${ }^{1}$. Additionally, few patient studies reported that Prevotellaceae family or Prevotella genus was less abundant in the PD patients ${ }^{17,18}$. We found that Prevotella bacterial levels were higher in younger TG rats compared with WT and that at an older age, bacterial levels were lower in TG PD rats, which is consistent with PD patient studies.

Furthermore, our current findings demonstrate that a-Syn expression leads to change in gut microbiota composition and more specifically the Lactobacillus and Alistipes genera in early young stage, non-symptomatic younger age versus older aged, symptomatic TG rats. 438 Abundance of Lactobacillus genera is in contrast ${ }^{17,22-26}$ with many PD patient studies, whilst 439 some are advocating in favour of this study ${ }^{18,27,28}$. Alistipes abundance was increased with 440 ageing in TG rats suggesting an increase in inflammation in the colon. Similarly, like rat PD 441 model, Alistipes was reported to be increased in PD patients ${ }^{28}$. Reports in other model 
442 system such as colon carcinoma and inflammatory bowel disease suggested that increased 443 abundance of Alistipes could lead to enhanced inflammatory phenotype in these models ${ }^{52-54}$. 444 It appears that Alistipes could be a slow inducer of inflammation in the colon with ageing TG 445 rats, however, further study is warranted. Moreover, our metabolites and 446 immunophenotyping data highlighted enhanced inflammation comes with ageing in TG rats. 447 Lactobacillus is considered anti-inflammatory bacteria ${ }^{55,56}$, subsequently there could be a 448 possibility that a low abundance of this bacteria allows a niche for the development of more 449 opportunistic inflammatory bacteria such Alistipes and Desulfovibrio genera. Additionally, a450 Syn overexpression could be key in governing the abundance of Lactobacillus in 451 homozygous TG rats. Our maternal microbiome study could explain a decreased abundance 452 of Lactobacillus as heterozygous mothers have an equivalent percentage of WT, while 453 homozygous TG rats have less abundant Lactobacillus. Thus, the present data are implying 454 that genetics is a major driving force in shaping host-microbiome interactions in our PD rat 455 model. Further, in vivo validation with the individual gut bacterial genera/strain is required to 456 support this notion.

457 A major PD patho-histological hallmark is the occurrence of eosinophilic cytoplasmic 458 neuronal inclusions (Lewy bodies) as well as a-Syn positive neuronal processes called Lewy 459 neurites ${ }^{4,15,57}$. Immunohistochemical data from PD patients before the onset of disease (2-5 460 years) suggested that $a-S y n$ positive neurons were present in sigmoid colon mucosa ${ }^{15}$. Thus, 461 as suggested earlier, it is possible that inflammation-induced oxidative stress could lead to 462 misfolding of a-Syn in TG rats, and successively a-Syn pathology is spreading to the brain in 463 a prion-like fashion ${ }^{3,10,11,13,15,58}$. It is also conceivable that changes in intestinal bacteria over 464 the course of ageing lead to an increase in intestinal endotoxins, which could enhance the 465 local or systemic inflammation and induce oxidative stress leading to disruption of intestinal 466 barriers or enteric neuroinflammation ${ }^{30,59}$. In line with this, we found that ageing increased gut 467 and systemic inflammation in TG rats and several metabolites such as succinate, lactate, 468 glutamate and 4-hydroxyphenylacetate in the blood serum and feces of aged TG rats (a 469 systemic route of inflammation). It was recently reported that succinate was highly abundant 470 in urine from PD patients compared with healthy controls, furthermore, succinate was 471 correlated with motor score and hence disease severity ${ }^{60}$. Thus, succinate could be used as 472 a prognostic marker for PD pathology. Additionally, the blood serum levels of other anti473 inflammatory metabolites such as phenylacetate and tyrosine/tryptophan were decreased in 474 ageing TG rats. Our data suggest that with ageing, an inflammatory environment is indeed 475 be prominent. The inflammatory niche and dysregulated bacterial composition may 476 accelerate the accumulation or aggregation of phosphorylated $a$-Syn in the myenteric 477 plexuses of TG rats. This is in keeping with previously described results in the brain which 478 suggested that aged TG rats had higher accumulation of $\alpha-S y n^{3,36}$.

479 Several chronic autoimmune intestinal diseases such as inflammatory bowel disease, celiac 480 disease, type 1 diabetes, multiple sclerosis, and systemic lupus erythematosus are linked 481 with increased intestinal permeability also referred to as "leaky gut" ${ }^{161}$. Thus, gut leakiness in 482 patients with a genetic susceptibility to PD may be a pivotal early step promoting a pro483 inflammatory/oxidative environment contributing to the initiation and/or progression of the PD 484 process $^{30}$. In line with this, our Ussing chamber data highlighted that ageing TG rats tended 485 to have more intestinal permeability and significantly less salt entry into intestinal cells than 486 age-matched WT control rats. Thus, we postulate that a lower uptake to $\mathrm{Na}^{+}$ions from the 487 stool could have deleterious effects on Lactobacillus. This is in agreement with a recent 488 study, as increased salt intake reduced the Lactobacillus in mice model ${ }^{62}$. Furthermore, 
serum sodium levels are inversely associated with dyskinesia in Parkinson's disease patients as lower levels of serum sodium are more likely to have dyskinesia ${ }^{63}$. Nevertheless, further understanding the physiology of ion channels and the gut microbiome would be helpful to understand gut-related onset of PD pathology.

Recently several inflammatory proteins such as calprotectin was found to be upregulated in the feces of PD patients ${ }^{47,48}$. The other inflammatory proteins such MCP1 was also correlated with PD progression ${ }^{64}$ as well as in Parkinson's disease susceptible murine model DJ- $1^{33}$. Our recent data in PD murine model suggested that several inflammatory proteins were also be present in the feces of TG rats ${ }^{44}$. Similarly, we also found that in the rat model with ageing increases levels of calprotectin levels in the feces and serum. Our RNA-seq data pointed that several inflammatory genes such as NFAT regulation of immune response, neuroinflammation and Th1 immune response pathways were upregulated with ageing and reduction in PD-1, PD-L-1 immunomodulatory pathways, antioxidant pathways and LXR/RXR activation in TG rats. Further, these data were validated using immunophenotyping and found that activated $\mathrm{CD}^{+}{ }^{+} \mathrm{T}$ cells had increased expression of IFN- $\gamma$ in TG rats. IFN- $\gamma$ producing $\mathrm{T}$ cells involved in induction of inflammation in the brain and activate the inflammatory microglial cells in the SNpc region, thus, in turn leads to neuronal cell death and nitrated a-Syn ${ }^{65}$. Most importantly PD-1 inhibitors involved in neurological toxicities in patient with non-small-cell lung cancers ${ }^{66}$ as well as melanoma patient ${ }^{67}$. Thus, reduction in PD-L-1 pathways could also participate in neurodegeneration including PD as our RNA-seq data from colon tissues suggested that PD-1, PD-L-1 immunomodulatory pathways were drastically reduced in TG rats. However, further validation studies are warranted to understand these pathways in PD pathology.

Our microbiome manipulation study by use of broad-spectrum antibiotics again highlights the importance of the gut microbiome and, in particular its ability to modify metabolite production which in theory could be directly involved in the gut inflammation and, thus PD pathology. The antibiotics treatment completely abolished the SCFAs, reduced the succinate production, and increased the production of the amino acids; tyrosine/tryptophan. Tyrosine is the precursor of dopamine synthesis and tryptophan in serotonin production respectively ${ }^{68}$. Accordingly, it could be conceivable that gut bacteria could be regulating the succinate, tyrosine and tryptophan production and could influence neurodegenerative processes by regulating dopamine and serotonin production. Furthermore, it could also be plausible that gut microbiome manipulation by short-term antibiotics treatment could help to modify metabolite production due to dysregulated gut microbiome in PD patients which might improve PD symptoms. However, further detailed investigations are required before it could be implemented for PD patients.

In summary, overall, our TG rat model was able to detect changes in the gut microbiota, intestinal permeability, a-Syn aggregation in parallel with ageing. These characteristics can similarly be detected in human PD patients. A previous study from our lab also suggested that this model exhibits early alterations in avoidance and novelty-seeking behaviour and late motor decline in TG rats ${ }^{36}$ where most of the bacterial diversity and intestinal permeability are drastically changed. Furthermore, our studies showed that a-Syn aggregation could be detected within the walls of the colon of TG rats before the brain pathology develops.

\section{Material and Methods}

\section{Animals}


534 The BAC-SNCA TG rats have been described in detail earlier and these animals were kept 535 maintaining on outbred conditions on Sprague Dawley background ${ }^{36}$. The rats were housed 536 3-4 per cage under a $12 \mathrm{~h}$ light-dark cycle with ad libitum access to food and water. All 537 experiments were performed according to the EU Animals Scientific Procedures Act and the 538 German law for the welfare of animals. All procedures were approved (TVA: HG3/18) by the 539 authorities of the state of Baden-Württemberg.

540 Antibiotics treatment

541 WT and TG rats (8-10 weeks age) were treated with a cocktail of antibiotics 542 amoxicillin/clavulanic acid $(4: 1 ; 0.5 \mathrm{~g} / \mathrm{L})$, Vancomycin $(0.5 \mathrm{~g} / \mathrm{L})$, Neomycin $(0.5 \mathrm{~g} / \mathrm{L})$ Cefuroxime $543(0.5 \mathrm{~g} / \mathrm{L})$, Streptomycin $(5 \mathrm{~g} / \mathrm{L})$ and ampicillin $(0.5 \mathrm{~g} / \mathrm{L})$ in drinking water containing $(5 \%$ 544 sucrose). Rats were kept in IVCs one week before the treatment started and till the end of 545 the experiments. Further during the treatment with antibiotics, rats were monitored twice per 546 day for their health and safety (body weight, water content and visual inspection). All the 547 antibiotics treated rats were healthy, a slight reduction of body weight in the beginning of the 548 treatment was observed, however, after one week there was no difference in body weight. 549 Antibiotics were given in an increasing dose (first $0.25 \mathrm{~g} / \mathrm{L}$ and after 3 days it was switched to $5500.5 \mathrm{~g} / \mathrm{L}$ until 2 weeks of treatment). The reason for this was to acclimatize the rats for the 551 antibiotics as of the initial concentration of $0.5 \mathrm{~g} / \mathrm{L}$, the rats did not drink the water and started 552 to lose weight due to dehydration. Once antibiotics were given in lower concentration $553(0.25 \mathrm{~g} / \mathrm{L})$ the rats started to drink properly and after 3 days they were switched to a $0.5 \mathrm{~g} / \mathrm{L}$ 554 antibiotic cocktail.

\section{Bacterial DNA isolation}

556 Faecal pellets were collected from different aged of animals (one-month - 14 months old rats) 557 depending on the experiments for microbial diversity. To collect the faecal pellet, rats were 558 taken out from their home cages and individually placed into a new cage and waited until rats 559 excreted faecal pellet (normally 2-3 pellets were collected). Within 5 minutes of excretion, 560 faecal pellets were kept in cryotubes and transferred onto dry ice during the collection period 561 and after collection of all samples faecal pellets were kept at $-80^{\circ} \mathrm{C}$ until use.

562 Bacterial DNA was isolated using the QIAamp FAST DNA stool Mini Kit (\#51604, Qiagen, 563 Germany) as described by manufacture's recommendation. In brief, approximately 140-180 $564 \mathrm{mg}$ of faecal pellets was taken, crushed and kept in $2 \mathrm{ml}$ tubes for bacterial DNA isolation 565 (pathogen detection) and samples were kept on ice until all the feces were measured. After 566 measurement of all the feces, $1 \mathrm{ml}$ of InhibitEX buffer was added to each faecal containing 567 tube. Tubes were vortexed continuously until the faecal sample is thoroughly homogenized 568 and samples were kept at $70^{\circ} \mathrm{C}$ for 5 minutes and vortexed for 15 seconds after the 569 incubation. Samples were further centrifuged for full speed for 1 minute to pellet faecal 570 particles obtained from the previous step. $200 \mu \mathrm{l}$ buffer AL was added and samples were 571 vortexed for 15 seconds. This mixture was incubated at $70^{\circ} \mathrm{C}$ for 10 minutes. After 572 incubation, add $200 \mu \mathrm{l}$ of $100 \%$ ethanol was added to the lysate and mixed by vortexing. All 573 the lysate was loaded onto QIAamp spin column and centrifuged at full speed for 1 minute. 574 Each column then was washed with $500 \mu \mathrm{l}$ buffer AW1, AW2 and DNA was eluted in 100ul of 575 ATE buffer and DNA was stored at $-20^{\circ} \mathrm{C}$.

576 16S rRNA sequencing and data analysis 
577 To compare the diversity and composition of gut microbial community, we isolated bacterial 578 DNA from the feces of the WT and TG rats of the respective age (1M-14M) groups cohorts 579 and performed PCR of the 16S ribosomal RNA gene (variable region V3 and V4). The PCR 580 products were sequenced using next generation sequencing. Sequenced amplicons were 581 trimmed and analysed using bioinformatics tools.

582 For $16 \mathrm{~S}$ rRNA gene amplification, $12.5 \mathrm{ng}$ of DNA was amplified using $0.2 \mu \mathrm{M}$ of both forward 583 (TCGTCGGCAGCGTCAGATGTGTATAAGAGACAGCCTACGGGNGGVWGCAG) and 584 reverse

585 (GTCTCGTGGGCTCGGAGATGTGTATAAGAGACAGGACTACHVGGGTATCTAATCC;

586 both from Metabion) and KAPA HiFi HotStart Ready Mix (\#KK2601, KAPABiosystems). PCR

587 was performed using a first denaturation of $95^{\circ} \mathrm{C}$ for 3 minutes, followed by 25 cycles of 588 amplification at $95^{\circ} \mathrm{C}$ for 30 seconds, $55^{\circ} \mathrm{C}$ for 30 seconds and $72^{\circ} \mathrm{C}$ for 30 seconds, final 589 elongation at $72^{\circ} \mathrm{C}$ for 5 minutes and the amplified DNA was stored at $4^{0} \mathrm{C}$. DNA 590 electrophoresis of samples was used to validate the amplicon specificity.

591 Samples were then subjected to Agencourt AMPure XP PCR purification system (Beckman 592 Coulter) utilizing Agencourt's solid-phase paramagnetic bead technology for high-throughput 593 purification of PCR amplicons. Agencourt AMPure XP utilizes an optimized buffer to 594 selectively bind PCR amplicons $100 \mathrm{bp}$ and larger to paramagnetic beads. Excess primers, 595 nucleotides, salts, and enzymes can be removed using a simple washing procedure. The 596 resulting purified PCR product is essentially free of contaminants. Further, PCR amplicons 597 were indexed using Nextera XT DNA Library Prep Kit and KAPA HiFi HotStart ReadyMix. 598 PCR was performed using first denaturation of $95^{\circ} \mathrm{C}$ for 3 minutes, followed by 8 cycles of 599 amplification at $95^{\circ} \mathrm{C}$ for 30 seconds, $55^{\circ} \mathrm{C}$ for 30 seconds and $72^{\circ} \mathrm{C}$ for 30 seconds, final 600 elongation at $72^{\circ} \mathrm{C}$ for 5 minutes. Samples were purified and then validated using 601 BioAnalyzer (Bioanalyzer DNA1000, Agilent) and 4nM of each library pooled using unique 602 induces before sequencing on a MiSeq (Illumina) and paired 300 bp reads.

603 The obtained sequence reads were sorted by the unique barcodes and sequences of the 604 barcode, linker, and primers were removed from the original sequencing reads using Fastx 605 toolkit. Sequence reads were aligned using MALT (MEGAN alignment tool) for SILVA in 606 semi-global mode and with percent identity threshold of $90 \%$. Further analysis and 607 visualization were performed using MEGAN-CE as described earlier ${ }^{44}$.

\section{Shotgun sequencing}

609 Isolated DNA was quantified using Qubit (Thermofisher) and presented comparable optical 610 density quality ratio, concentration $26-75 \mathrm{ng} / \mu \mathrm{l}$ and fragment size distribution. Samples 611 were normalized to $0.5 \mathrm{ng} / \mu \mathrm{l}$ in Tris $(10 \mathrm{mM})$ and an input of $1 \mathrm{ng}$ was processed following 612 Illumina's Nextera library preparation protocol. DNA was tagmentated for 5 minutes at $55^{\circ} \mathrm{C}$. 613 The resulting DNA was then amplified and indexed using 12 PCR enrichment cycles and a 614 dual combination of barcode primers. After amplification, DNA libraries clean-up was 615 performed using AMPure XP bead purification and were resuspended in resuspension buffer. 616 Resulting libraries presented similar molarity $11-25 \mathrm{nmol}$ and were pooled based on their 617 molarity in hybridization buffer and denatured following the Nextera XT protocol. Libraries 618 were sequenced using the Illumina NextSeq HighOutput using 2x 150 bp paired end 619 sequencing, providing $34-41$ million clusters per sample.

620 Measurement of $\mathrm{Na}^{+}$Current and gut permeability using an 'Ussing Chamber' 
621 ENaC activity was estimated from the amiloride-sensitive potential difference and current 622 across the rat colonic epithelium as described in mouse epithelium layer ${ }^{69}$. After removing 623 the outer serosal and the muscular layer under a microscope, tissues were mounted onto a 624 custom-made 'mini-Ussing chamber' with an opening diameter of $0.99 \mathrm{~mm}$ and an opening 625 area of $0.00769 \mathrm{~cm}^{2}$. The serosal and luminal perfusate contained (in $\mathrm{mM}$ ): $145 \mathrm{NaCl}, 1$ $626 \mathrm{MgCl}$, $2.6 \mathrm{Ca}$-gluconate, $0.4 \mathrm{KH} 2 \mathrm{PO} 4,1.6 \mathrm{~K} 2 \mathrm{HPO} 4,5$ glucose. To assess ENaC induced 627 current, $50 \mu \mathrm{M}$ amiloride (Sigma, in DMSO) was added to the luminal perfusate. ENaC 628 activity was estimated from the effect of ENaC blocker amiloride $(50 \mu \mathrm{M})$ on the 629 transepithelial potential difference and current, which reflects the $\mathrm{Na}^{+}$reabsorption from the 630 colon epithelial layer. Transepithelial potential difference (Vte) was determined continuously 631 and transepithelial resistance (Rte) estimated from the voltage deflections ( $\Delta \mathrm{Vte}$ ) elicited by 632 imposing rectangular test currents of $1 \mu \mathrm{A}$ and $1.2 \mathrm{~s}$ duration at a rate of $8 / \mathrm{min}$. Rte was 633 calculated according to Ohm's law.

\section{Preparation of the colon for histological analysis}

635 The colon samples prepared on ice were fixed for $24 \mathrm{~h}$ in $4 \%$ PFA and then stored at $4^{0} \mathrm{C}$ in $6360.4 \%$ PFA. Colon samples were prepared from rats deeply anesthetized by $\mathrm{CO}_{2}$ inhalation. 637 Fixed colon samples were then alcohol-dehydrated and embedded in paraffin. Samples were 638 embedded in paraffin blocks using a tissue embedding station and stored at room 639 temperature. Paraffin blocks containing brains were cooled on ice block and cut in $7 \mu \mathrm{m}$ thick 640 sections using a microtome. Sections were placed in $45^{\circ} \mathrm{C}$ water bath for flattering, collected 641 on a glass slide, dried in an incubator at $50^{\circ} \mathrm{C}$ for $1 \mathrm{~h}$ and stored at room temperature.

642 Immunohistochemistry of the colon section

643 Sections were deparaffinized in xylene and rehydrated in decreasing ethanol. Optionally, a 644 digestion in $50 \mu \mathrm{g} / \mathrm{ml}$ of PK (315836, Roche) was performed in PK-buffer (10 mM Tris, 100 $645 \mathrm{mM} \mathrm{NaCl}$ and $0.1 \% \mathrm{NP}-40$ ) at $37^{\circ} \mathrm{C}$ for $10-60$ minutes to stain insoluble proteins. Heat 646 antigen retrieval was performed for 15 min using a microwave set at $1000 \mathrm{~W}$ and using 10 $647 \mathrm{mM}$ sodium citrate $(\mathrm{pH} \mathrm{6.0)}$ ) and sodium citrate was refilled every $5 \mathrm{~min}$ to avoid the slides to 648 dry. Sections were then washed 3 times 3 min in Tris buffer saline (TBS; Tris $1 \mathrm{M}, \mathrm{NaCl} 3 \mathrm{M}$ 649 and $\mathrm{pH} 7.5$ ) and incubated $20 \mathrm{~min}$ in $0.3 \%$ hydrogen peroxide to block endogenous 650 peroxidases. After washing the slides 3 times 3 min in TBS, non-specific binding was blocked 651 for $40 \mathrm{~min}$ by treating sections with $5 \%$ normal serum of the secondary antibody host animal 652 (goat serum: S-1000, Vector; rabbit serum: S-5000, Vector; donkey serum: 017-000-001, 653 Dianova) diluted in TBS and supplemented with $0.3 \%$ Triton X-100 to permeabilize cell 654 membranes. After washing the slides 3 times $3 \mathrm{~min}$ with TBS, sections were incubated 655 overnight with the primary antibody diluted in TBS containing $1 \%$ normal serum. The next 656 day, slides were washed 3 times $3 \mathrm{~min}$ with TBS+0.025\% Triton X-100 and incubated for $1 \mathrm{~h}$ 657 with a biotin conjugated secondary antibody (mouse: BA-9200, rabbit: BA-1000, rat: BA658 4000; Vector) diluted 1:250 in PBS containing 1\% normal serum. Sections were washed 3 659 times $3 \mathrm{~min}$ with TBS $+0.025 \%$ Triton X-100 and then incubated with an avidin-biotin 660 enhancer complex coupled with peroxidase (ABC Elite; Vector) at room temperature for $1 \mathrm{~h}$. 661 After washing 3 times 3 min with TBS+0.025\% Triton X-100, sections were detected using 662 3,3'-diaminobenzidine (DAB; Sigma) providing a brown precipitate when oxidized by the 663 peroxidase linked to the secondary antibody. Reaction was stopped by washing slides in 664 distilled water after $3 \mathrm{~min}$. For microscopy, slides were mounted using a cover slipping 665 media, dried overnight, and stored at room temperature. 
667 The colons for the mass spectrometry or Immunoblotting analysis were collected from freshly 668 euthanized rats and cleaned with PBS to remove faecal material and snap-frozen in liquid 669 nitrogen and samples were stored at $-80^{\circ} \mathrm{C}$ freezer until use. In certain experiments, 670 myenteric plexus and epithelial layer were also isolated and samples were stored at $-80^{\circ} \mathrm{C}$ 671 until use.

672 Proteins were lysed from the colon tissues in 10 volume of RIPA buffer ( $50 \mathrm{mM}$ Tris, $150 \mathrm{mM}$ $673 \mathrm{NaCl}, 1.0 \%$ NP-40, $0.5 \%$ sodium deoxycholate, $0.1 \%$ SDS, pH 8.0) supplemented with 674 protease inhibitor (Complete; Roche Diagnostics) in order to perform gel electrophoresis. 675 Brain tissues were disrupted $30 \mathrm{sec}$ using a homogenizer (T10 ultra turrax; VWR) in ice. 676 After the homogenization, samples were incubated for $30 \mathrm{~min}$ at $4^{\circ} \mathrm{C}$ and spun for $20 \mathrm{~min}$ at $67712000 x g$. Proteins lysate supernatants were supplemented with $10 \%$ glycerol before long 678 storage at $-80^{\circ} \mathrm{C}$.

679 Protein concentration was determined using BCA method (BCA Protein Assay Kit, 23225, 680 Life Technology) or Bradford assay. Samples were prepared by diluting protein lysates in 681 PAGE buffer (0.2 M glycine, $25 \mathrm{mM}$ Tris, $1 \%$ SDS), followed by a denaturation at $95^{\circ} \mathrm{C}$ for 10 $682 \mathrm{~min}$ in loading buffer (80 mM Tris, 2\% SDS, 5\% 2-mercaptoethanol, 10\% glycerol, $0.005 \%$ 683 bromophenol blue, $\mathrm{pH} 6.8$ ) and a short centrifugation $30 \mathrm{sec}$ at $400 \times \mathrm{g}$. Proteins were 684 separated by electrophoresis using 12\% SDS-PAGE gel. Gels containing proteins were 685 washed for 5 minutes in transfer buffer (0.2 M glycine, $25 \mathrm{mM}$ Tris, $10-20 \%$ methanol) and 686 transferred to membranes equilibrated in transfer buffer. Transfer was performed for $1 \mathrm{~h} 30$ 687 minutes at $80 \mathrm{~V}$ at $4^{\circ} \mathrm{C}$ on PVDF membranes. Immunoblot were washed 5 min in TBS buffer 688 and blocked using 5\% non-fat milk (Slim Fast) in TBS. Membranes were then washed twice $6895 \mathrm{~min}$ in Tris buffer saline containing $0.01 \%$ Triton X-100 (TBST) and incubated with the 690 primary antibody over night at $4^{\circ} \mathrm{C}$ (human and mouse a-Syn: 610786 BD Biosciences). After 691 incubation with the first antibody, membranes were washed four times (5 min each) with 692 TBST. Membranes were then incubated for $75 \mathrm{~min}$ with the secondary antibody coupled to 693 horseradish peroxidase (GE Healthcare). After four washing steps with TBST (5 min each), 694 bands were visualized using the enhanced chemiluminescence method (ECL+; GE 695 Healthcare). Light signal was detected using LI-COR Odyssey and were quantified using 696 Odyssey software.

697 ELISA

To measure the calprotectin/MRP 8/14 from the fecal samples, S100A8/S100A9 ELISA kit (\#K6936, Immundiagnostik AG) was used according to the manufacture's guidelines. First faecal samples were measured (weight $50 \mathrm{mg}$ ) and dissolved in $500 \mathrm{ul}$ of extraction buffer supplied by the kit, mixed by vortexing and then centrifuged for $10 \mathrm{~min}$ at $3000 \mathrm{xg}$. Supernatant was taken and transferred to a new microcentrifuge tube and $100 \mu$ of sample was used for measuring the protein. The data were analyzed using 4 parameters algorithm and the concentration of calprotectin was normalized with feces weight and data are presented in $\mathrm{ng} / \mathrm{g}$.

RNA-sequencing from the colon tissues 
710 TissueLyser II (Qiagen). RNA isolation was performed on the QIAsymphony (Qiagen) 711 following the platform Standard protocol. Elution was performed using $50 \mu \mathrm{l}$ of RNase-free 712 water.

713 RNA quality was assessed with an Agilent 2100 Bioanalyzer and the Agilent RNA 6000 Nano 714 kit (Agilent). 3'RNA-sequencing was performed using $100 \mathrm{ng}$ of total RNA and the QuantSeq 715 3' mRNA-Seq (\#015.24; Lexogen, Austria). Libraries were sequenced on the NextSeq500 716 using the Mid Output v2.5 150 Cycles (\#20024904, Illumina) with a depth of $>2$ millions reads 717 each.

718 FASTQ were generated using fastp (v0.20.0) and RNA-seq data quality was assessed to 719 identify potential issues with sequencing cycles, low average quality, adaptor contamination, or repetitive sequences from PCR amplification. Reads were aligned using STAR (v2.7.2a) against a custom-built genome composed of the Ensembl Mus musculus grcm38. Alignment quality was analyzed using MappingQC (v1.8) and visually inspected in the Integrative Genome Viewer (v2.4.19). Normalized read counts for all genes were obtained using edgeR (v3.26.6). Transcripts covered with less than 4 count-per-million in at least 1 sample were excluded from the analysis leaving $>14,000$ genes for determining differential expression in each of the pair-wise comparisons between experimental groups.

RNA sequencing data was further subjected to Ingenuity pathway analysis (IPA) to identify the genes involved in different canonical pathways as well as with reactome online tool.

Flow cytometry

Plasma cells were first washed with PBS at $600 \times \mathrm{xg}$ for 5 minutes at $4{ }^{0} \mathrm{C}$. Supernatant was discarded, and cells were first stained with surface staining antibodies (CD4, CD8, CD25, CD44) then were fixed with Foxp3 fixation/permeabilization buffer (eBioscience, Germany) for intracellular staining and incubated for 30 minutes. After incubation, cells were washed with $1 \mathrm{x}$ permeabilization buffer, exposed to added intracellular monoclonal antibodies (Foxp3, IL-17 and IFN-g in different channel) and incubated for an additional 45 minutes.

736 Cells were washed again with permeabilization buffer and PBS was added to acquire the cells on a flow cytometer (BSFortessa ${ }^{\text {TM }}$ from Becton Dickinson; Heidelberg, Germany).

Tunica muscularis strips were fixed with $4 \%(\mathrm{w} / \mathrm{v})$ phosphate buffered $\mathrm{p}$-formaldehyde (PFA; Sigma-Aldrich, St. Louis, MO, USA) for $20 \mathrm{~min}$ and rinsed three times with phosphate buffered saline (PBS). Antigen retrieval was carried out in citrate buffer $(0.01 \mathrm{~mol} / \mathrm{l}, \mathrm{pH} 6.0$, Roth, Karlsruhe, Germany) by heating the samples in a microwave (600 W for $5 \mathrm{~min}$ ) and successively allowed the samples cool overnight. To prevent unspecific binding of antibodies, samples were blocked for two days at room temperature with PBS containing $4 \%$ (v/v) goat serum (Biochrom, Berlin, Germany), $0.1 \%(\mathrm{v} / \mathrm{v})$ bovine serum albumin (BSA; Roth), $746 \quad 0.3 \%(\mathrm{v} / \mathrm{v})$ Triton $\AA \mathrm{X}-100$ (Roth), and 0.1\% (wt/v) $\mathrm{NaN}_{3}$ (Merck, Darmstadt, Germany) on a shaker. Afterwards, samples were incubated with primary antibody diluted in PBS with $0.1 \%$ $(\mathrm{v} / \mathrm{v})$ BSA, $0.3 \%(\mathrm{v} / \mathrm{v}) \operatorname{Triton}^{\circledR} \mathrm{X}-100$, and $0.1 \%(\mathrm{wt} / \mathrm{v}) \mathrm{NaN}_{3}$ for three days at room temperature. Samples were then rinsed in PBS with $0.1 \%(\mathrm{wt} / \mathrm{v}) \mathrm{NaN}_{3}$ three times, with the last washing step over night. Then secondary antibodies were diluted in PBS with $0.1 \%(\mathrm{v} / \mathrm{v})$

751 BSA, $0.3 \%(\mathrm{v} / \mathrm{v})$ Triton® $\mathrm{X}-100$, and $0.1 \%(\mathrm{wt} / \mathrm{v}) \mathrm{NaN}_{3}$ and incubated overnight. Samples were then washed three times in PBS, immersed in $80 \%(\mathrm{v} / \mathrm{v})$ glycerol (Sigma-Aldrich) in 
754 The following primary antibodies were used for immunocytochemistry: mouse anti- $\alpha$ 755 Synuclein (1:500, BD, Franklin Lakes, NJ, USA), rabbit anti- $\beta$-III tubulin (TUJ, 1:400, 756 BioLegend, San Diego, CA, USA). Primary antibodies were detected using the fluorescent 757 secondary antibodies goat anti-rabbit Alexa 488 (1:400, Invitrogen, Carlsbad, CA, USA) and goat anti-mouse Alexa 546 (1:400, Invitrogen). Nuclear staining was carried out with DAPI solution (200 ng/ml, Roth). A Zeiss Axio Imager.Z1 fluorescence microscope with integrated Apotome module (Zeiss, Jena, Germany) was used for microscopic evaluation and structured illumination imaging. Images were acquired using Zeiss Zen blue software (Zeiss).

762 Feces and serum sample preparation for the metabolite detection using ${ }^{1} \mathrm{H}-\mathrm{NMR}$

763

764

765

766

767

768

769

770

771

772

773

774

775

776

For metabolite extraction and separation from lipids that disturb the ${ }^{1} \mathrm{H}-\mathrm{NMR}$ spectra of polar metabolites, a standard 2-phase extraction protocol was used. Hereby, $50 \mathrm{mg}$ of deep-frozen feces sample were transferred into $2 \mathrm{ml}$ adaptive focused acoustics (AFA) glass tubes (Covaris Inc, Woburn, USA) and mixed with $400 \mu \mathrm{l}$ of ultrapure methanol and $800 \mu \mathrm{L}$ of Methyl ter-butyl ether (MTBE; solvent grade). The mixture was manually dispersed with a disposable plastic spatula, then vortexed and transferred to a focused ultrasonicator (Covaris E220evolution, Woburn, USA). Feces metabolites were extracted with a 5 min lasting ultrasonication program in a degassed water bath at $7^{\circ} \mathrm{C}$. After extraction, the metabolite suspension was separated into a polar and lipid phase by adding $400 \mu \mathrm{l}$ of ultrapure water. In order to remove any remaining solids from the samples, the glass tubes were centrifuged for $5 \mathrm{~min}$ at $4000 \mathrm{xg}$. $700 \mu \mathrm{l}$ of polar upper phase was then transferred to a fresh $1.5 \mathrm{ml}$ eppendorf tube. The polar phase was subject to a 2nd centrifugation step for $5 \mathrm{~min}$ at $12,000 \times \mathrm{xg}$ and $600 \mu \mathrm{l}$ of the supernatant were transferred to a new $1.5 \mathrm{ml}$ eppendorf tube and evaporated to dryness over night with a vacuum concentrator (Eppendorf Speedvac).

We prepared the serum samples for NMR analysis using following method. Briefly, $45 \mu \mathrm{l}$ of serum and $90 \mu \mathrm{l}$ of $100 \%$ methanol was used and then mixed with the Covaris 130 microTUBE glass vials for metabolites extractions (no phase separation was used). This procedure was done for 25 minutes (5 minutes/samples) in one cycle. Once the mixing was done, $135 \mu \mathrm{l}$ of sample mixture supernatant was collected in a fresh $500 \mu \mathrm{l}$ tube and subjected to centrifuge for 30 minutes at $12,000 \times$ g. After centrifugation, $120 \mu$ of supernatant was taken in a fresh $1.5 \mathrm{ml}$ Eppendorf tube and subjected to speed vacuum for the overnight and dried pellets was dissolved in $50 \mu \mathrm{l}$ NMR buffer (recipe+STDs) was added and briefly sonicated to mix the buffer and metabolite pellets. Mixed solution $45 \mu \mathrm{l}$ of the supernatant were transferred with gel loading pipette tips into $1.7 \mathrm{~mm}$ NMR tubes (Bruker BioSpin, Karlsruhe, Germany) and a 96 well rack placed into the cooled $\left(4^{\circ} \mathrm{C}\right)$ NMR autosampler.

For the feces samples, we used a slightly different protocol for NMR analysis. The dried faecal pellets (after overnight speedvac) were resuspended with $60 \mu \mathrm{l}$ of deuterated phosphate buffer (200 mM K2HPO4, $200 \mu \mathrm{M} \mathrm{NaN3}$, pH 7.4) containing $1 \mathrm{mM}$ of the internal standard TSP (trimethylsilylpropanoic acid). For a maximum dissolution, the plastic tubes were quickly sonicated and then centrifuged $5 \mathrm{~min}$ at $14,000 \mathrm{xg} .50 \mu \mathrm{l}$ of the supernatant were transferred with gel loading pipette tips into $1.7 \mathrm{~mm}$ NMR tubes (Bruker BioSpin, Karlsruhe, Germany) and a 96 well rack placed into the cooled $\left(4^{\circ} \mathrm{C}\right)$ NMR autosampler.

Spectra were recorded on a $600 \mathrm{MHz}$ ultra-shielded NMR spectrometer (Avance III, Bruker BioSpin $\mathrm{GmbH}$ ) equipped with a triple resonance $\left({ }^{1} \mathrm{H},{ }^{13} \mathrm{C},{ }^{31} \mathrm{P}\right) 1.7 \mathrm{~mm}$ room temperature probe at $298 \mathrm{~K}$. For optimum water suppression and shim adjustment a quick simple ZG experiment was performed followed by a 1hour lasting CPMG (Carr-Purcell-Meiboom-Gill) 
experiment in order to suppress residual background signals from macromolecules such as bilirubin (time domain $=64 \mathrm{k}$ points, sweep width $=20 \mathrm{ppm}, 512$ scans). The recorded free induction decays (FIDs) were fourier-transformed and spectra properly phase- and baseline corrected (Bruker Topspin 3.5.6). The hereby created files were processed with ChenomX NMR Suite 8.3 for metabolite annotation and quantification. For statistical analysis, different grouped metabolite concentration tables were created and imported with MetaboAnalyst 4.0. In detail, we investigated both the effect of ageing (between 3 and 14 months) as well as genotype (WT and TG) on the metabolic phenotypes in serum and feces.

807 Statistical analysis

MEGAN-CE (version 6.14.2, built 23 Jan 2019) and MicrobiomeAnalyst were used for data acquisition and analysis. GraphPad and Inkscape were used for the final figure preparation. One-way ANOVA, Two-way ANOVA (post-hoc Sidak's test) or Student's t-test was used for statistical analysis using GraphPad (version 8) wherever it was appropriate and described in the figure legend. Data shown in violin plots which represents the median and quartiles as the box-and-whisker plots and it also display a smoothed frequency distribution. The $p$ value $(\leq 0.05)$ considered significant. Metabolite concentrations from ${ }^{1} \mathrm{H}-\mathrm{NMR}$ analysis were exported as comma separated value spreadsheet file to MetaboAnalyst, normalized with PQN (probabilistic quantile normalization) to account for dilution effects and pareto scaled for making metabolites within one sample comparable. A combined fold change (FC > 1.2) and Student's t-test analysis ( $p<0.05)$ was used for the volcano and box plots. Furthermore, multivariate heat maps, PLS-DA plots and VIP scores were generated for direct comparison of the genotypes at $3 \mathrm{M}$ and $>12 \mathrm{M}$ in feces and serum, respectively. The effect of antibiotics treatment on serum and feces metabolite profiles was investigated by heat maps, sPLS-DA and VIP scores.

\section{Declarations}

\section{Ethics statement and approval}

All the experiments were performed according to the EU Animals Scientific Procedures Act (2010/63/EU) and the German law for the welfare of the animals. All the procedure and methods were approved by the local government authorities (Regierungspräsidium, Tübingen; TVA HG3/18) of the state of Baden-Württemberg, Germany.

\section{Consent for publication}

No patients or human data was used in this study. All authors read the manuscript and approved to be co-authors on the manuscript and have substantial contribution in the manuscript.

\section{Availability of data and material}

The datasets used and/or analysed during the current study are available from the corresponding authors on a reasonable request. RNA-seq data are available from GEO accession no. xxx.

\section{Competing interests}

The authors declare that they have no competing interests. 
840 This research project is an EU Joint Programme - Neurodegenerative Disease Research 841 (JPND) (JPCOFUND_FP-829-047 aSynProtect) and is supported through the funding 842 organization Deutschland, Bundesministerium für Bildung und Forschung (BMBF, FKZ). This 843 research was also supported by the Deutsche Forschungsgemeinschaft, DFG through the 844 funding of the NGS Competent Centre Tübingen (NCCT-DFG). Funders have no role in the 845 study design and data analysis.

846 Acknowledgements

847 We thank Prof Bernd Pichler for allowing to have access to high-field ${ }^{1} \mathrm{H}-\mathrm{NMR}$ spectroscopy 848 for metabolomics studies. We acknowledge Prof. Jeroen Raes, VIB-KU Leuven Center for 849 Microbiology, Rega Institute, Leuven, Netherlands for helpful discussion with microbiome 850 data and calprotectin assay development. We would like to acknowledge the support from 851 FACS core facility and NGS Competence Centre Tübingen (NCCT). We also acknowledge 852 the helpful discussion with aSynProtect team members (Jia-Yi Li, Tiago Outeiro, Olaf Riess, 853 Pekka Kallunki, Daniel Otzen, Richard Wade-Martins, Jeroen Raes, Hilal Lashuel). We 854 acknowledge support by Deutsche Forschungsgemeinschaft (DFG) and Open Access 855 Publishing Fund of University of Tübingen.

\section{Author's contribution}

857 YS: Study design, performed the research and managed the overall project, involved in 858 entire study, analyzed the data, made the figures, and wrote the manuscript.

CT: Performed the metabolites study, data analysis, made the figures, wrote the manuscript

JR, HAL, K-HS: performed the characterization of a-Syn from the ENS of the rat colon, expression of a-Syn in whole-mount staining and analysed the data.

AD, JA: Helped with $16 \mathrm{~S}$ rRNA data analysis bioinformatics meta data analysis and made the figures.

MSS, FL: performed the Ussing chamber experiments and analysed the data.

PHN: Performed the whole-mount staining procedure and microscopy for antibiotic treatment experiments.

867 IF, MA, NW: performed the Immunoblotting experiments from colon and brain samples and 868 data analysis.

MDS, DO: Study design for microbiome sequencing and data analysis.

NC, OR: Provided tools for the study, original microbiome study design, data analyses, discussion, edited the manuscript and funding acquisition.

\section{Fig. legends}

Fig.1 The gut microbiome dynamics with ageing, increased Lactobacillus and decreased

874 Alistipes bacterial genera in the BAC-hSNCA TG rats. (a) Experimental design for the 875 microbiome study. To understand the microbiome dynamics with ageing, heterozygous 876 female and heterozygous male were used for breeding to keep the same gut microbiome 
877 from the mother to avoid maternal effect. When females were pregnant, male were removed 878 from the cage and pubs were allowed to stay with the mothers for 3 weeks or until weaning. 879 Three weeks old pubs were genotyped and then separated into either WT or homozygous 880 BAC-SNCA transgenic groups (TG; refers to always homozygous until unless it is stated 881 elsewhere) groups then faecal sample collections were started at the age of 1 month (4-5 882 weeks age) onwards $(2,2.5,3,6,>12(12-14))$ for the microbiome analysis by either $16 \mathrm{~s}$ 883 rRNA gene amplicon or shotgun sequencing methods. The animals were kept in 3-4 different 884 cages to avoid the 'cage affect' bias in data analysis. Both male and females were used for 885 the faecal sample collection depending on the breeding. (b) Representation of bacterial 886 diversity (hollow pie chart) at phylum levels in WT and TG rats. (c) Firmicutes/Bacteroidetes 887 (F/B) ratio was calculated for WT and TG rat samples and significant difference was 888 observed at $2.5 \mathrm{M}$ and $>12 \mathrm{M}$ age. $\mathrm{F} / \mathrm{B}$ ratio was lower in $\mathrm{TG}$ rats at $2.5 \mathrm{M}$ age however, this 889 ratio was reversed at $>12 \mathrm{M}$ which was significantly higher in TG rats. Student's t-test was 890 performed for comparisons at a given age for WT and TG. Two-way ANOVA was performed 891 to find the significance between different time points and genotypes. (d) The microbiome 892 dynamics at genera level was estimated. Data are represented in colour-coded stacked-bar 893 chart for each age group for the WT and TG rats. Each bar chart represents the \% 894 abundance of bacterial genera of the total bacteria in each group at particular age. 895 Significant change in bacterial phyla is shown in asterisk for particular phyla together with $p$ 896 value signficance. (e,f) The dynamic representation of Lactobacillus and Alistipes with ageing 897 in WT and TG respectively. A significant difference was observed with ageing in 898 Lactobacillus and Alistipes. Two-way ANOVA was performed to find the significance between 899 different time points and genotypes. $P$ value significance represents ${ }^{*} p \leq 0.05,{ }^{* *} p \leq 0.01$ and $900{ }^{* * *} p \leq 0.001$.

901 Fig. 2 The gut dysbiosis in ageing TG rats. (a) To find out the bacterial dysbiotic bacterial 902 strains, we have performed shotgun DNA sequencing and calculated the abundance of 903 bacterial phyla (hollow pie charts) at $>12 \mathrm{M}$. (b) F/B ratio was significantly higher in TG 904 compared with WT. (c, d) Several bacterial genera were significantly upregulated 905 (Desulfovibrio, Alistipes, Flavonifractor, Oscillibacter, Paenibacillus, Clostridium and 906 Butyrivibrio) and significantly downregulated (Streptococcus, Bacteroidetes, Lactobacillus, 907 Parabacteroidetes, and Prevotella) at $>12 \mathrm{M}$ age in TG rats. (e) Clustering of gut bacteria into 908 three major clusters from WT and TG rats. (f) Alpha diversity at genera level was calculated 909 using Shannon-Weaver index and found to be significantly lower diversity in TG rats 910 compared with WT. (g) Beta diversity was estimated for both the genotypes of rats with 911 Principal component analysis (PCoA) using Bray-Curtis method and both WT and TG rats 912 cluster differently (PC1 54.5\% and PC2 15.8). $P$ value significance represents ${ }^{*} p \leq 0.05$.

913 Fig. 3 Ageing affects the succinate, tryptophan and tyrosine metabolites in the feces and 914 serum. Metabolites combined fold change $(F C>1.2(\log 2 F C))$ and $p$-value $<0.05(-\log 10(p$ 915 value)) volcano plot analysis identified effect of ageing between WT and TG rat feces and 916 serum. (a) At 3M age feces genotype comparison TG/WT phenylacetate is significantly 917 upregulated whereas at $>12 \mathrm{M}$ age 4-Hydroxyphenylacetate and lactate were up while 918 glutamate down. (b) In serum samples glutamate is significantly decreased at 3M whereas at $919>12 \mathrm{M}$ succinate, glutamate and lactate are increased. (c) When only observing the ageing 920 effect on TG serum metabolites, succinate is significantly upregulated and succinate 921 correlation coefficient analysis suggested that tryptophan and tyrosine were negatively 922 correlated with succinate while other short chain fatty acids were positively correlated. 
923 Statistically significant levels showed in the respective volcano and violin plots. $P$ value 924 significance represents ${ }^{*} p \leq 0.05,{ }^{* *} p \leq 0.01$ and ${ }^{* * *} p \leq 0.001$.

Fig. 4 Accumulation of $a-S y n$ in the colon of TG rats. (a) IHC was performed to identify the accumulation of total human a-Syn in 2, 2.5, 3 and $>12 \mathrm{M}$ old WT and TG rats using human primary a-Syn antibody (LB509; \#ab27766). With ageing accumulation of a-Syn was observed in TG rats whereas no positive staining was observed in WT rats as expected. (b) Further, pathological state of human $\alpha$-Syn was examined in 2, 2.5, 3 and $>12 \mathrm{M}$ in TG rats using human primary p-129 a-Syn antibody (EP1356Y; \#ab51253). It appeared that pathological a-Syn was increased with ageing in TG rats. Further, p-129 a-Syn antibody was blocked using specific peptide for $\mathrm{p}-129$ site for the antibody and no staining was observed suggesting the specificity of p-129 antibody. (c) One of the representatives immunoblot images of a-Syn and Gapdh staining's are shown. We quantified the a-Syn amount using total $\alpha$-Syn antibody (\#BD 610787) and found that with ageing (2M) to 4M $\alpha$-Syn expression in the colon tissues is significantly increased. Further ageing ( $4 \mathrm{M}$ to $>12 \mathrm{M})$ tended to affect 937 the accumulation of $\alpha$-Syn but not statistically significant difference was observed. $P$ value 938 significance represents ${ }^{*} p \leq 0.05$.

Fig. 5 Increased permeability and transepithelial current in TG rats. (a) A schematic diagram 940 showing the 'Ussing chamber' technique to measure the function of intestinal permeability 941 and $\mathrm{Na}+$ uptake to gut epithelial cells (Enac channel). (b) Original tracing illustrating the effect of currents $(1 \mu \mathrm{A})$ and of amiloride $(50 \mu \mathrm{M})$ on transepithelial potential across colonic epithelium from WT (upper) and TG (lower) from 2 and 12M rats. (c) Arithmetic mean +/SEM ( $n=3-5 /$ group) of amiloride-sensitive current across colonic epithelium ( $\mathrm{Na}^{+}$absorption) from TG and WT rats and resistance of colonic epithelium (permeability). (d) Faecal hypoxanthine levels measured by ${ }^{1} \mathrm{H}-\mathrm{NMR}$ from $3 \mathrm{M}$ and $>12 \mathrm{M}$ WT and TG rats. $\mathrm{P}$ value significance represents ${ }^{*} p \leq 0.05,{ }^{* *} p \leq 0.01,{ }^{* *} p \leq 0.001$ and ${ }^{* * *} p \leq 0.001$.

948 Fig. 6 Increased inflammatory signals in the colon mucosa and submucosa (MS layer) of $>12$ $949 M$ TG rats. (a) Fecal/serum calprotectin levels measured by ELISA in 2, 8 and $>12$ M WT and $\mathrm{TG}$ rats. (b) $\mathrm{WT}$ and $\mathrm{TG}(3 \mathrm{M}$ and $>12 \mathrm{M}$ old) rat colon mucosa and submucosa (gut epithelium) was taken and subjected to RNA-seq. (b,c) Ingenuity pathway analysis (IPA) from TG and WT rats showed upregulation of several biological pathways in $>12 \mathrm{M} \mathrm{TG}$ rats. (d) The heatmap shows upregulation (yellow) and downregulation (red) genes in the gut MS layer. TG rats have higher expression of various genes involved in inflammation compared with WT. (e) IPA analysis in $>12 \mathrm{M} \mathrm{TG}$ rats suggested that several inflammatory pathways were changed. (f) FACS plots show increase in numbers of $\mathrm{CD} 4^{+} \mathrm{T}$ cells, CD $4^{+} \mathrm{CD} 25^{+} \mathrm{T}$ cells (activated), $\mathrm{CD}^{+} \mathrm{CD} 44^{+} \mathrm{T}$ cells (memory) and $\mathrm{CD} 4^{+} \mathrm{IFN}-\mathrm{\gamma}^{+} \mathrm{T}$ cells were significantly higher in 958 the blood of $>12 \mathrm{M}$ TG rats compared with WT. Bar plots show the means $+/-$ SEM. $P$ value 959 significance represents ${ }^{*} \mathrm{p} \leq 0.05$.

960 Fig. 7 Treatment with broad-spectrum antibiotics leads to reduced gut microbiome load, no 961 change in a-Syn expression and reduced level of succinate metabolites. (a) CFU 962 measurement after broad-spectrum antibiotics treatment in WT and TG rats after equal 963 amount of feces. (b) Amount of total DNA after antibiotics treatment in WT and TG rat faecal 964 samples. (c) Antibiotics affected the bacterial phyla differently for WT and TG rats. (d) F/B 965 ratio was decreased after antibiotic treatment in WT and TG rats respectively, however, no 966 significance difference was observed. (e) Alpha diversity measurement at genera level after 967 antibiotics treatment for WT and TG rats. (f) Beta diversity measurement (genera level) using 968 Bray-Curtis method. Antibiotics treatments affect the clustering of intestinal bacteria. (g) 
969 Immunoblot image of a-Syn expression in ENS and olfactory bulb brain region from control 970 and antibiotics treated TG rats and quantification of immunoblots are shown in violin plots. 971 (h) Whole mount staining of muscular layer (longitudinal, circular and myenteric muscle 972 layer) and expression of neuronal (Tuj) and a-Syn expression. (i) Heat-map presenting the 973 RNA-seq data from control and antibiotics treated $3 \mathrm{M}$ rats. (j) Succinate, tryptophan and 974 tyrosine levels in the feces and serum from control and antibiotics treated WT and TG (3M) 975 rats. $P$ value significance represents ${ }^{*} p \leq 0.05$.

\section{Suppl. Figures}

977 Suppl. Fig. 1 The gut microbiome dynamics with ageing, increased Lactobacillus and 978 decreased Alistipes bacterial genera in the TG rats. (a) 16S rRNA gene amplicon sequencing 979 was performed on samples from 1, 2, 2.5, 3, 6 and $14 \mathrm{M}$ age group WT and TG respectively. 980 Total number of sequences read obtained from WT and TG samples were similar and a 981 significant difference was not observed. (b) The alpha diversity within each group (WT and 982 TG rat) was estimated using Shannon-Weaver index based on phylum level classifications. 983 No significant difference was noticed in between WT and TG rats at any age group. Data are 984 represented in Box and Whisker plots. (c) Age dynamics of phyla Firmicutes, Bacteroidetes, 985 Actinobacteria, Proteobacteria and Verrucomicrobia. Each phylum was significantly different 986 at particular age as shown in the figure with significant level. (d) The Shannon-Weaver index 987 and Chao 1 (alpha diversity) at genus level at particular age. Significant change is shown in 988 asterisk for a defined age group. (e) The dynamic representation of Prevotella, Alloprevotella, 989 Parabacteroides, Alistipes Lactobacillus, Turicibacter, Ruminococcus, Desulfovibrio and 990 Bifidobacterium with ageing in WT and TG respectively. A significant difference is shown for 991 a particular age group. $P$ value significance represents ${ }^{*} p \leq 0.05,{ }^{* *} p \leq 0.01$ and ${ }^{* * *} p \leq 0.001$.

992 Suppl. Fig. 2 Clustering of bacterial phyla for different age group WT and TG rats. Bacterial 993 clustering figure showed change in the bacterial abundance with ageing.

994 Suppl. Fig. 3 Genetic makeup of animal could be an important factor for microbiome 995 composition development. (a) A study was carried out from 4M old heterozygous females 996 (total no of female (F) 6; 2 females/cage (C); colour coded) were kept in 3 different cages 997 and when females were pregnant then male were separated. Faecal samples were collected 998 from the females after pups were born. After 3 weeks of age, pups were separated from 999 females and genotyped and kept in WT and homozygous TG groups in 5 different cages $1000(\mathrm{C} 1-5)$ as explained in the fig. Faecal samples were sequenced by 16S rRNA sequencing 1001 and analysed by MEGAN-CE software. Clustering analysis at species level suggested that 1002 WT and homozygous TG rats' clusters differently with each other as well as with mother's 1003 (Heterozygous) microbiome. (b) The percentage abundance of Lactobacillus genus and 1004 species in two different facilities at the same age group in two different cohorts. $P$ value 1005 significance represents ${ }^{*} \mathrm{p} \leq 0.05$.

1006 Suppl. Fig. 4 Similar presence of Lactobacillus genus in two different facilities. (a) At 3M age 1007 species comparison study showed that two different facilities had similar abundance of Lactobacillus species. (b) The percentage abundance of Lactobacillus genus and species in two different facilities at the same age group in two different cohorts.

1010 Suppl. Fig. 5 Coprophagy is one of route and method for microbial manipulation in PD rat sequence reads. (c) Hollow pie charts represent the bacterial composition at phylum level 
1014 charts show the bacterial composition before and after faecal transplant in WT and TG PD 1015 rat model. (f) Bacterial composition in individual WT and TG rats before and after transplant 1016 and represented as heat-map.

1017 Suppl. Fig. 6 The gut microbiome dynamics with ageing, increased Lactobacillus and 1018 decreased Alistipes bacterial genera in TG rats. (a) Total no of sequence reads after shotgun 1019 sequencing obtained from WT an TG samples were similar. (b) Rarefaction plots for all 1020 samples. (c) Clustering of bacterial at species level from WT and TG rats (>12M). (d) 1021 Significantly changed bacterial species in WT and TG rats.

1022 Suppl. Fig. 7 The metabolic profiles of 3M feces (a) and serum (c) show no clear separation 1023 of WT and TG rats in the PLS-DA scores plots and also heat maps show no clear clusters. 1024 By contrast, after $>12 \mathrm{M}$ TG and WT samples could be clearly separated in the PLS-DA 1025 scores plots in both feces (b) and serum (d) analysis. The most prominent metabolites in the 1026 VIP scores analysis for feces samples were succinate (high in TG for 3M) and glutamate 1027 (high in WT for $>12 \mathrm{M}$ ). For serum, 3M rats showed high glucose levels in TG and high lactate 1028 in WT, while at $>12$ M TG rats showed highly increased lactate and succinate levels.

1029 Suppl. Fig. 8 The serum metabolome analysis of WT rats $(a)$ revealed significant $(p<0.05)$ 1030 metabolite fold changes ( $F C>1.2$ ) during ageing. At $>12 \mathrm{M}$ of lifetime, WT serum were 1031 composed with increased levels of formate, isoleucine, valine, taurine, creatine, glutamine 1032 and lysine and decreased values of betaine, N,N-dimethylglycine, glutamate, sn-glycero-31033 phosphocholine, citrate and 3-hydroxybutyrate. Succinate was increased at 3 months of age, 1034 however above the significance threshold. Results from the same spectra with multivariate 1035 PLS-DA VIP scores analysis (c) provided similar results for WT while TG rats showed mainly an in increase in lactate and succinate. A comparison of the feces metabolome during aging showed both for WT and TG a decrease in succinate levels (b) for which a correlation analysis was performed.

1039 Suppl. Fig. 9 Fragmented $\alpha$-Syn and expression in ENS from the colon of $>12 M$ TG rats. (a) 1040 Estimation of fragmentation in a-Syn in from the colon ENS. Two major truncated fragments 1041 were found in ENS as shown in a-Syn immunoblot image. (b) Expression of a-Syn in the 1042 colon muscular layer.

1043 Suppl. Fig. 10 Accumulation of a-Syn in TG rat colon. Colon tissues were digested with 1044 trypsin for 2 hours (1:5 dilution of the original) and performed with human (TG rats) and rat 1045 (WT rats) specific antibodies as described in materials and methods. IHC showed the 1046 accumulation of a-Syn in both 2M and 18M TG rats, whereas in WT (for both the age group) 1047 a-Syn was dissolved by trypsin so it appeared that a-Syn in WT existed in soluble form 1048 whereas a-Syn was retained in TG rats suggested the accumulation.

1049 Suppl. Fig. 11 Human a-Syn is truncated in TG rats. (a) The expression of endogenous a1050 Syn in rat colon and upto 4 different truncated a-Syn can be observed in the colon of the TG 1051 rats, but the appearance of these different bands differs between individual and does not 1052 seem to be correlated with age. In some TG rats (5M 1 and 18M 1), the expression of a-Syn 1053 is similar than in the WT rats but showing truncated fragments of lower molecular weight. No 1054 pathologic phosphorylation (S129, Y125, Y39) could be detected by immunoblotting method. 1055 (b). DSP treatment increases a-Syn immunodetection in WB. The detection of the truncated 1056 a-Syn band is not dependent on boiling the samples, as similar band is also present in non1057 boiled samples, but its signal is increased in presence of the crosslinker DSP. (c) Using 1058 different $a$-Syn antibodies covering different epitopes of the sequence were used to detect 
1059 the fragmentation of a-Syn. N-terminal antibodies did not detect the first truncated fragment 1060 present in sample 1 and 2- Detect the second truncated fragment present in sample 2 and 3. 1061 Detect an extra truncated fragment in sample 1 with a similar molecular weight the second 1062 truncated fragment. C-terminal antibodies do not detect the first and second truncated 1063 fragment present in sample 1 and 2, but only upto residue 125. SA3400 (117-131) does not 1064 detect these truncated forms. The extra truncated fragment in sample 1 is detected by all c1065 terminal antibodies. $1^{\text {st }}$ truncated band is observed with a-Syn 211 (121-125), 14H2L1 (1171066 125), LB509(115-122) and FL140 (61-95), however it was not observed with SA3400 (1171067 131), EP1466Y (N-term), N19 (N-term) and Ab6176 (11-26). The fact that the $1^{\text {st }}$ truncated 1068 band was not observed by SA3400 (117-131), however it was observed by all the other C1069 term antibodies upto 125, suggest that it might be a $\mathrm{N}$-terminal fragment with the truncation 1070 located between the position 125 and 131. Further, the truncated fragment was not observed 1071 by any of the three $\mathrm{N}$-term antibodies tested, thus further suggesting that the fragment was 1072 also truncated at $\mathrm{N}$-terminal part. The $2^{\text {nd }}$ band was observed with $\mathrm{N} 19$ (N-term), Ab6176 1073 (11-26), EP1646Y (N-term), 14H2L1 (117-125) and LB509 (115-122) and not observed with 1074 SA3400 (117-131). Thus, detected band was observed by all the $\mathrm{N}$-term antibodies but not 1075 by the most distant C-term antibody (SA3400) suggesting that it was truncated in the C1076 terminal part, probably around the position 120. (d) A cartoon is showing possible aberrant 1077 alternative splicing variant sites.

1078 Suppl. Fig. 12 RNA-seq analysis from 3M and $>12 \mathrm{M}$ old WT and TG colon epithelial tissues. 1079 (a) Differentially expressed genes at 3M age in WT and TG rats. Total no of differentially 1080 expressed genes (153) based on absolute log fold-change of at least 1 (raw $p$ value $P=0.05$ ), 1081 whereas at $>12 \mathrm{M}$ age total no of differentially expressed were 1808 based on absolute log 1082 fold-change of at least 1 (raw $p$ value $P=0.05$ ). There was no statistical significance based on 1083 false discovery rate (FDR) for 3M, however at $>12 \mathrm{M} 264$ genes were different. (b) Clustering 1084 of $>12 \mathrm{M}$ WT and TG epithelial (Epi) samples. (c,d), IPA analysis of adaptive (Th1 pathway) 1085 and innate (TLR pathway) for the $>12 \mathrm{M}$ samples. (e) Different inflammatory gene 1086 expressions

1087 Suppl. Fig. 13 Reactome transcriptome wide overview analysis of $3 \mathrm{M}$ and $>12 \mathrm{M}$ epithelial 1088 RNA-seq samples. The figure shows a genome-wide overview of the results of pathway 1089 analysis (a) $3 \mathrm{M}$ and (b) $>12 \mathrm{M}$. Reactome pathways are arranged in a hierarchy. The center 1090 of each of the circular "bursts" is the root of the one top-level pathway. Each step away from 1091 the center presents the next level lower in the pathway hierarchy. The colour code denotes 1092 over-representation of a particular pathway in the input dataset. Light grey signifies pathways 1093 which are not significantly over-presented. At 3M programmed cell death pathway genes 1094 were over-presented whereas at $>12 \mathrm{M}$ programmed cell death pathways gene were 1095 downregulated. Further, at $>12 \mathrm{M} \mathrm{M}$ there were an over-representation of immune system genes and less representation of programmed cell death genes.

1097 Suppl. Fig. 14 Antibiotics treatment increase TRPV1 and Dnase1 in the colon. (a) Clustering 1098 of the samples in the control (WT and TG) and antibiotics treated groups. (b) Genotype 1099 analysis in control and antibiotics treated colon samples (epi). Total 165 genes were found to 1100 be commonly regulated by antibiotics. More differential gene expression in WT compared 1101 with TG rats. (c) Expression of TRPV1, Dnase1 in WT and TG colon.

1102 Suppl. Fig. 15 Metabolites changes in the feces and serum after antibiotics treatment at 3M 1103 age. (a) Antibiotics treatment results in a clear separation of feces samples in the (a) PLS-DA 1104 analysis (b) and heat map. (c) While the effect on the corresponding serum scores plots and 
1105 (d) heat map is rather small. (e,f,g) Multivariate VIP scores analysis identified decreased 1106 succinate in both TG and WT control samples after antibiotics treatment alongside the 1107 ketone body 3-Hydroxybutyrate. Box and whisker plots showed the succinate and 31108 Hydroxybutyrate in control and antibiotic treated samples.

\section{References}

11101 Keshavarzian, A. et al. Colonic bacterial composition in Parkinson's disease. Mov

2 Antunes, L. et al. Similar alpha-Synuclein staining in the colon mucosa in patients with Parkinson's disease and controls. Mov Disord 31, 1567-1570, doi:10.1002/mds.26702 (2016).

3 Zhong, C. B. et al. Age-Dependent Alpha-Synuclein Accumulation and Phosphorylation in the Enteric Nervous System in a Transgenic Mouse Model of Parkinson's Disease. Neurosci Bull 33, 483-492, doi:10.1007/s12264-017-0179-1 (2017).

4 Kalia, L. V. \& Lang, A. E. Parkinson's disease. The Lancet 386, 896-912, doi:10.1016/s0140-6736(14)61393-3 (2015).

5 Liddle, R. A. Parkinson's disease from the gut. Brain Res 1693, 201-206, doi:10.1016/j.brainres.2018.01.010 (2018).

6 Takahashi, H. \& Wakabayashi, K. Controversy: is Parkinson's disease a single disease entity? Yes. Parkinsonism Relat Disord 11 Suppl 1, S31-37, doi:10.1016/j.parkreldis.2005.02.011 (2005).

7 Chandra, R., Hiniker, A., Kuo, Y. M., Nussbaum, R. L. \& Liddle, R. A. alpha-Synuclein in gut endocrine cells and its implications for Parkinson's disease. $\mathrm{JCl}$ Insight 2, doi:10.1172/jci.insight.92295 (2017).

8 Holmqvist, S. et al. Direct evidence of Parkinson pathology spread from the gastrointestinal tract to the brain in rats. Acta Neuropathol 128, 805-820, doi:10.1007/s00401-014-1343-6 (2014).

9 Pfeiffer, R. F. Gastrointestinal Dysfunction in Parkinson's Disease. Curr Treat Options Neurol 20, 54, doi:10.1007/s11940-018-0539-9 (2018).

10 Visanji, N. P., Brooks, P. L., Hazrati, L. N. \& Lang, A. E. The prion hypothesis in Parkinson's disease: Braak to the future. Acta Neuropathol Commun 1, 2, doi:10.1186/2051-5960-1-2 (2013).

11 Braak, $\mathrm{H}$. et al. Staging of brain pathology related to sporadic Parkinson's disease. Neurobiol Aging 24, 197-211 (2003).

12 Derkinderen, P. et al. Parkinson disease: the enteric nervous system spills its guts. Neurology 77, 1761-1767, doi:10.1212/WNL.0b013e318236ef60 (2011).

13 Hawkes, C. H., Del Tredici, K. \& Braak, H. Parkinson's disease: a dual-hit hypothesis. Neuropathol Appl Neurobiol 33, 599-614, doi:10.1111/j.1365-2990.2007.00874.x (2007).

14 Goldman, J. G. \& Postuma, R. Premotor and nonmotor features of Parkinson's disease. Curr Opin Neurol 27, 434-441, doi:10.1097/WCO.0000000000000112 (2014).

15 Shannon, K. M., Keshavarzian, A., Dodiya, H. B., Jakate, S. \& Kordower, J. H. Is alpha-synuclein in the colon a biomarker for premotor Parkinson's disease? Evidence from 3 cases. Mov Disord 27, 716-719, doi:10.1002/mds.25020 (2012).

16 Horsager, J. et al. Brain-first versus body-first Parkinson's disease: a multimodal imaging case-control study. Brain 143, 3077-3088, doi:10.1093/brain/awaa238 (2020).

17 Scheperjans, F. et al. Gut microbiota are related to Parkinson's disease and clinical phenotype. Mov Disord 30, 350-358, doi:10.1002/mds.26069 (2015).

18 Unger, M. M. et al. Short chain fatty acids and gut microbiota differ between patients with Parkinson's disease and age-matched controls. Parkinsonism Relat Disord 32, 66-72, doi:10.1016/j.parkreldis.2016.08.019 (2016). 
115819 Scheperjans, F. Gut microbiota, 1013 new pieces in the Parkinson's disease puzzle.

1159

1160

1161

1162

1163

1164

1165

1166

1167

1168

1169

1170

1171

1172

1173

1174

1175

1176

1177

1178

1179

1180

1181

1182

1183

1184

1185

1186

1187

1188

1189

1190

1191

1192

1193

1194

1195

1196

1197

1198

1199

1200

1201

1202

1203

1204

1205

1206

1207

1208

1209

1210

1211

1212

Curr Opin Neurol 29, 773-780, doi:10.1097/WCO.0000000000000389 (2016).

20 Haikal, C., Chen, Q. Q. \& Li, J. Y. Microbiome changes: an indicator of Parkinson's disease? Transl Neurodegener 8, 38, doi:10.1186/s40035-019-0175-7 (2019).

21 Jackson, A. et al. Diet in Parkinson's Disease: Critical Role for the Microbiome. Front Neurol 10, 1245, doi:10.3389/fneur.2019.01245 (2019).

22 Hasegawa, S. et al. Intestinal Dysbiosis and Lowered Serum LipopolysaccharideBinding Protein in Parkinson's Disease. PLoS One 10, e0142164, doi:10.1371/journal.pone.0142164 (2015).

23 Hill-Burns, E. M. et al. Parkinson's disease and Parkinson's disease medications have distinct signatures of the gut microbiome. Mov Disord 32, 739-749, doi:10.1002/mds.26942 (2017).

24 Hopfner, F. et al. Gut microbiota in Parkinson disease in a northern German cohort. Brain Res 1667, 41-45, doi:10.1016/j.brainres.2017.04.019 (2017).

25 Petrov, V. A. et al. Analysis of Gut Microbiota in Patients with Parkinson's Disease. Bull Exp Biol Med 162, 734-737, doi:10.1007/s10517-017-3700-7 (2017).

26 Lin, C. H. et al. Altered gut microbiota and inflammatory cytokine responses in patients with Parkinson's disease. J Neuroinflammation 16, 129, doi:10.1186/s12974019-1528-y (2019).

27 Qian, Y. et al. Detection of Microbial 16S rRNA Gene in the Blood of Patients With Parkinson's Disease. Front Aging Neurosci 10, 156, doi:10.3389/fnagi.2018.00156 (2018).

$28 \mathrm{Li}, \mathrm{C}$. et al. Gut Microbiota Differs Between Parkinson's Disease Patients and Healthy Controls in Northeast China. Front Mol Neurosci 12, 171, doi:10.3389/fnmol.2019.00171 (2019).

29 Sampson, T. R. et al. Gut Microbiota Regulate Motor Deficits and Neuroinflammation in a Model of Parkinson's Disease. Cell 167, 1469-1480 e1412, doi:10.1016/j.cell.2016.11.018 (2016).

30 Forsyth, C. B. et al. Increased intestinal permeability correlates with sigmoid mucosa alpha-synuclein staining and endotoxin exposure markers in early Parkinson's disease. PLoS One 6, e28032, doi:10.1371/journal.pone.0028032 (2011).

31 Mulak, A. \& Bonaz, B. Brain-gut-microbiota axis in Parkinson's disease. World J Gastroenterol 21, 10609-10620, doi:10.3748/wjg.v21.i37.10609 (2015).

32 Yoo, B. B. \& Mazmanian, S. K. The Enteric Network: Interactions between the Immune and Nervous Systems of the Gut. Immunity 46, 910-926, doi:10.1016/j.immuni.2017.05.011 (2017).

33 Singh, Y. et al. DJ-1 (Park7) affects the gut microbiome, metabolites and the development of innate lymphoid cells (ILCs). Sci Rep 10, 16131, doi:10.1038/s41598020-72903-w (2020).

34 Huuskonen, J., Suuronen, T., Nuutinen, T., Kyrylenko, S. \& Salminen, A. Regulation of microglial inflammatory response by sodium butyrate and short-chain fatty acids. $\mathrm{Br}$ J Pharmacol 141, 874-880, doi:10.1038/sj.bjp.0705682 (2004).

35 Vascellari, S. et al. Gut Microbiota and Metabolome Alterations Associated with Parkinson's Disease. mSystems 5, doi:10.1128/mSystems.00561-20 (2020).

36 Nuber, S. et al. A progressive dopaminergic phenotype associated with neurotoxic conversion of alpha-synuclein in BAC-transgenic rats. Brain 136, 412-432, doi:10.1093/brain/aws358 (2013).

37 Krashia, P. et al. Blunting neuroinflammation with resolvin D1 prevents early pathology in a rat model of Parkinson's disease. Nat Commun 10, 3945, doi:10.1038/s41467-019-11928-w (2019).

38 Van Den Berge, $N$. et al. Evidence for bidirectional and trans-synaptic parasympathetic and sympathetic propagation of alpha-synuclein in rats. Acta Neuropathol 138, 535-550, doi:10.1007/s00401-019-02040-w (2019).

39 Jovel, J. et al. Characterization of the Gut Microbiome Using 16S or Shotgun Metagenomics. Front Microbiol 7, 459, doi:10.3389/fmicb.2016.00459 (2016). 
121340 Morgan, X. C. \& Huttenhower, C. Chapter 12: Human microbiome analysis. PLoS

1214 Comput Biol 8, e1002808, doi:10.1371/journal.pcbi.1002808 (2012).

41 Dhariwal, A. et al. MicrobiomeAnalyst: a web-based tool for comprehensive statistical, visual and meta-analysis of microbiome data. Nucleic Acids Res 45, W180-W188, doi:10.1093/nar/gkx295 (2017).

42 Huson, D. H. et al. MEGAN Community Edition - Interactive Exploration and Analysis of Large-Scale Microbiome Sequencing Data. PLoS Comput Biol 12, e1004957, doi:10.1371/journal.pcbi.1004957 (2016).

43 Bullich, C., Keshavarzian, A., Garssen, J., Kraneveld, A. \& Perez-Pardo, P. Gut Vibes in Parkinson's Disease: The Microbiota-Gut-Brain Axis. Mov Disord Clin Pract 6, 639651, doi:10.1002/mdc3.12840 (2019).

44 Singh Y, E.-H. M., Admard J, Wassouf J, JSchulze-Hentrich J, Ursula Kohlhofer, Quintanilla-Martinez L, Huson D, Riess O, Casadei N. Enriched environmental conditions modify the gut microbiome composition and fecal markers of inflammation in Parkinson's disease. Front Neurosci, doi:doi: 10.3389/fnins.2019.01032 (2019).

45 Lee, J. S. et al. Hypoxanthine is a checkpoint stress metabolite in colonic epithelial energy modulation and barrier function. J Biol Chem 293, 6039-6051, doi:10.1074/jbc.RA117.000269 (2018).

46 Houser, M. C. et al. Stool Immune Profiles Evince Gastrointestinal Inflammation in Parkinson's Disease. Mov Disord 33, 793-804, doi:10.1002/mds.27326 (2018).

47 Schwiertz, A. et al. Fecal markers of intestinal inflammation and intestinal permeability are elevated in Parkinson's disease. Parkinsonism Relat Disord 50, 104107, doi:10.1016/j.parkreldis.2018.02.022 (2018).

48 Mulak, A., Koszewicz, M., Panek-Jeziorna, M., Koziorowska-Gawron, E. \& Budrewicz, S. Fecal Calprotectin as a Marker of the Gut Immune System Activation Is Elevated in Parkinson's Disease. Front Neurosci 13, 992, doi:10.3389/fnins.2019.00992 (2019).

49 Pereira, P. A. B. et al. Oral and nasal microbiota in Parkinson's disease. Parkinsonism Relat Disord 38, 61-67, doi:10.1016/j.parkreldis.2017.02.026 (2017).

50 Mulak, A. A controversy on the role of short-chain fatty acids in the pathogenesis of Parkinson's disease. Mov Disord 33, 398-401, doi:10.1002/mds.27304 (2018).

51 Nguyen, T. L., Vieira-Silva, S., Liston, A. \& Raes, J. How informative is the mouse for human gut microbiota research? Dis Model Mech 8, 1-16, doi:10.1242/dmm.017400 (2015).

52 Moschen, A. R. et al. Lipocalin 2 Protects from Inflammation and Tumorigenesis Associated with Gut Microbiota Alterations. Cell Host Microbe 19, 455-469, doi:10.1016/j.chom.2016.03.007 (2016).

53 Yang, Y. \& Jobin, C. Novel insights into microbiome in colitis and colorectal cancer. Curr Opin Gastroenterol 33, 422-427, doi:10.1097/MOG.0000000000000399 (2017).

54 Dai, Z. et al. Multi-cohort analysis of colorectal cancer metagenome identified altered bacteria across populations and universal bacterial markers. Microbiome 6, 70, doi:10.1186/s40168-018-0451-2 (2018).

55 Ayyanna, R., Ankaiah, D. \& Arul, V. Anti-inflammatory and Antioxidant Properties of Probiotic Bacterium Lactobacillus mucosae AN1 and Lactobacillus fermentum SNR1 in Wistar Albino Rats. Front Microbiol 9, 3063, doi:10.3389/fmicb.2018.03063 (2018).

56 Morshedi, M., Hashemi, R., Moazzen, S., Sahebkar, A. \& Hosseinifard, E. S. Immunomodulatory and anti-inflammatory effects of probiotics in multiple sclerosis: a systematic review. J Neuroinflammation 16, 231, doi:10.1186/s12974-019-1611-4 (2019).

57 Spillantini MG, S. M., Lee VM, Trojanowski JQ, Jakes R, Goedert M. Alpha-synuclein in Lewy bodies. Nature 28, 839-840 (1997).

58 Lashuel, H. A., Overk, C. R., Oueslati, A. \& Masliah, E. The many faces of alphasynuclein: from structure and toxicity to therapeutic target. Nat Rev Neurosci 14, 3848, doi:10.1038/nrn3406 (2013).

59 Santos, S. F., de Oliveira, H. L., Yamada, E. S., Neves, B. C. \& Pereira, A., Jr. The Gut and Parkinson's Disease-A Bidirectional Pathway. Front Neurol 10, 574, doi:10.3389/fneur.2019.00574 (2019). 
126960 Kumari, S. et al. Identification of potential urine biomarkers in idiopathic parkinson's

1270

1271

1272

1273

1274

1275

1276

1277

1278

1279

1280

1281

1282

1283

1284

1285

1286

1287

1288

1289

1290

1291

1292

1293

1294

1295 disease using NMR. Clin Chim Acta 510, 442-449, doi:10.1016/j.cca.2020.08.005 (2020).

61 Mu, Q., Kirby, J., Reilly, C. M. \& Luo, X. M. Leaky Gut As a Danger Signal for Autoimmune Diseases. Front Immunol 8, 598, doi:10.3389/fimmu.2017.00598 (2017).

62 Wilck, N. et al. Salt-responsive gut commensal modulates TH17 axis and disease. Nature 551, 585-589, doi:10.1038/nature24628 (2017).

63 Mao, C. J. et al. Serum sodium and chloride are inversely associated with dyskinesia in Parkinson's disease patients. Brain Behav 7, e00867, doi:10.1002/brb3.867 (2017).

64 Santaella, A. et al. Cerebrospinal fluid monocyte chemoattractant protein 1 correlates with progression of Parkinson's disease. NPJ Parkinsons Dis 6, 21, doi:10.1038/s41531-020-00124-z (2020).

65 Campos-Acuna, J., Elgueta, D. \& Pacheco, R. T-Cell-Driven Inflammation as a Mediator of the Gut-Brain Axis Involved in Parkinson's Disease. Front Immunol 10, 239, doi:10.3389/fimmu.2019.00239 (2019).

66 Mirabile, A. et al. PD-1 Inhibitors-Related Neurological Toxicities in Patients with NonSmall-Cell Lung Cancer: A Literature Review. Cancers (Basel) 11, doi:10.3390/cancers11030296 (2019).

67 Johnson, D. B. et al. A case report of clonal EBV-like memory CD4(+) T cell activation in fatal checkpoint inhibitor-induced encephalitis. Nat Med 25, 1243-1250, doi:10.1038/s41591-019-0523-2 (2019).

68 Daubner, S. C., Le, T. \& Wang, S. Tyrosine hydroxylase and regulation of dopamine synthesis. Arch Biochem Biophys 508, 1-12, doi:10.1016/j.abb.2010.12.017 (2011).

69 Salker, M. S. et al. LEFTYA Activates the Epithelial $\mathrm{Na}+$ Channel (ENaC) in Endometrial Cells via Serum and Glucocorticoid Inducible Kinase SGK1. Cell Physiol Biochem 39, 1295-1306, doi:10.1159/000447834 (2016). 
Heterozygous SNCA-TG

(3.0 M old mothers)
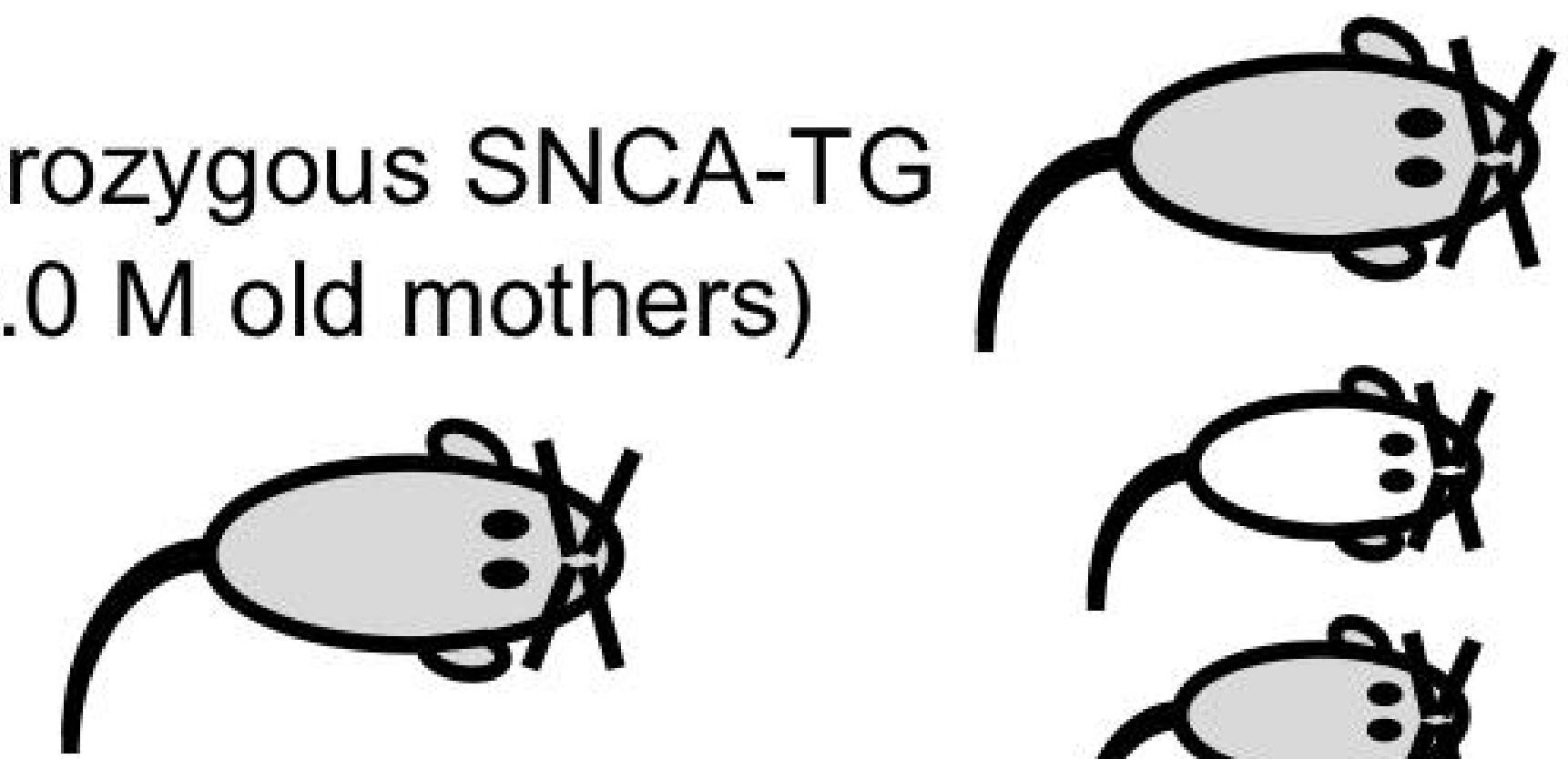

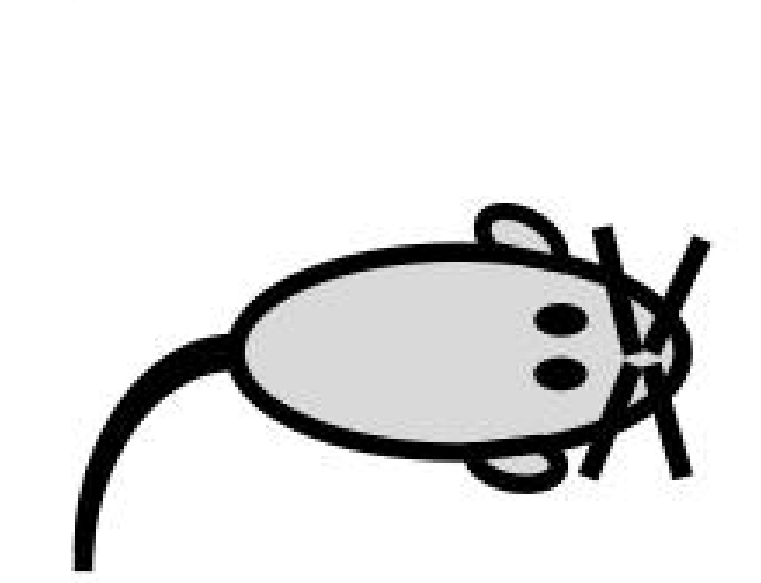

Heterozygous SNCA-TG

(3.0 M old father)
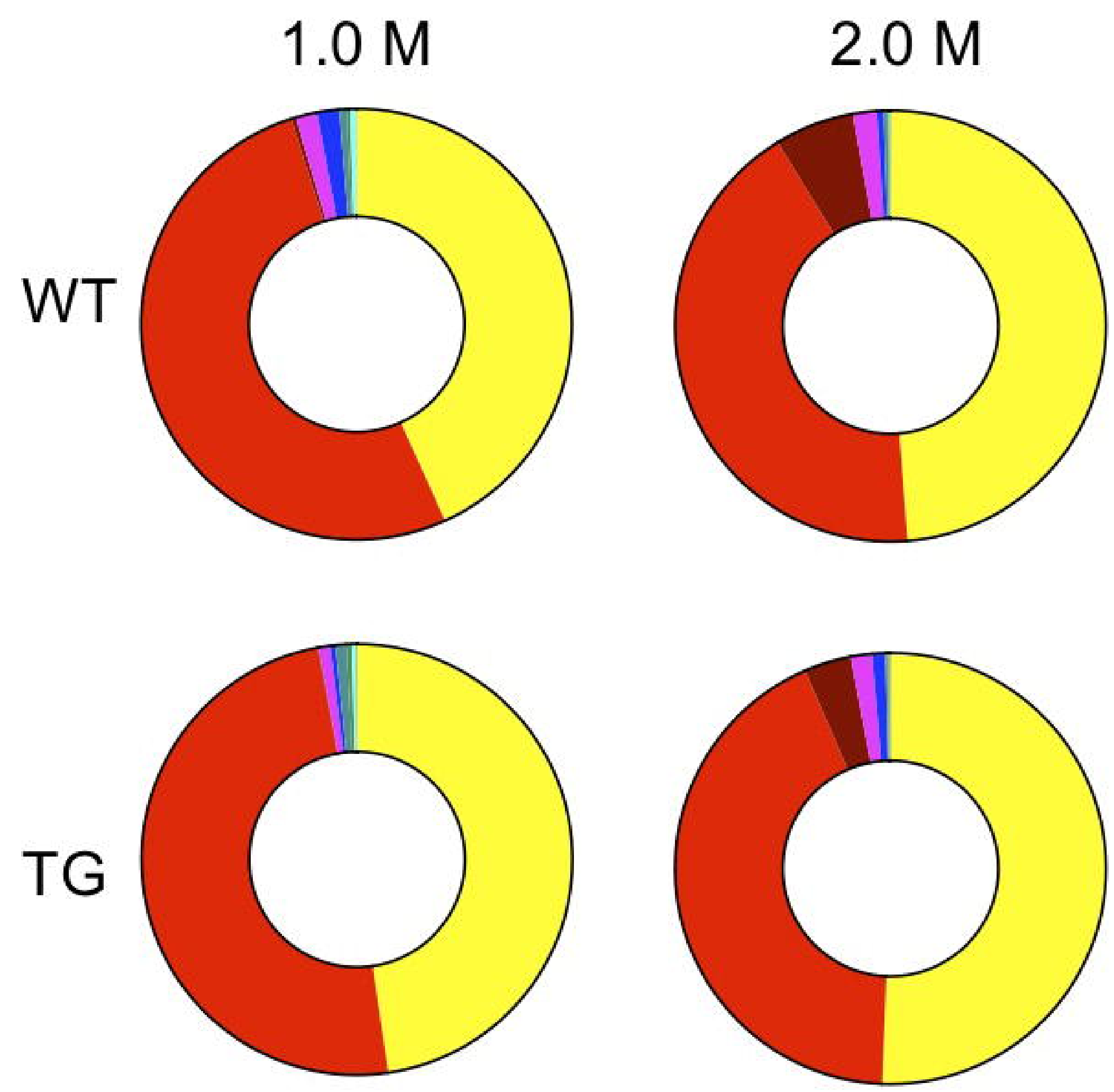

Proteobacteria

$\left({ }^{*} \mathrm{p}=0.04\right)$

Aquificae $\left({ }^{* *} \mathrm{p}=0.007\right)$

C

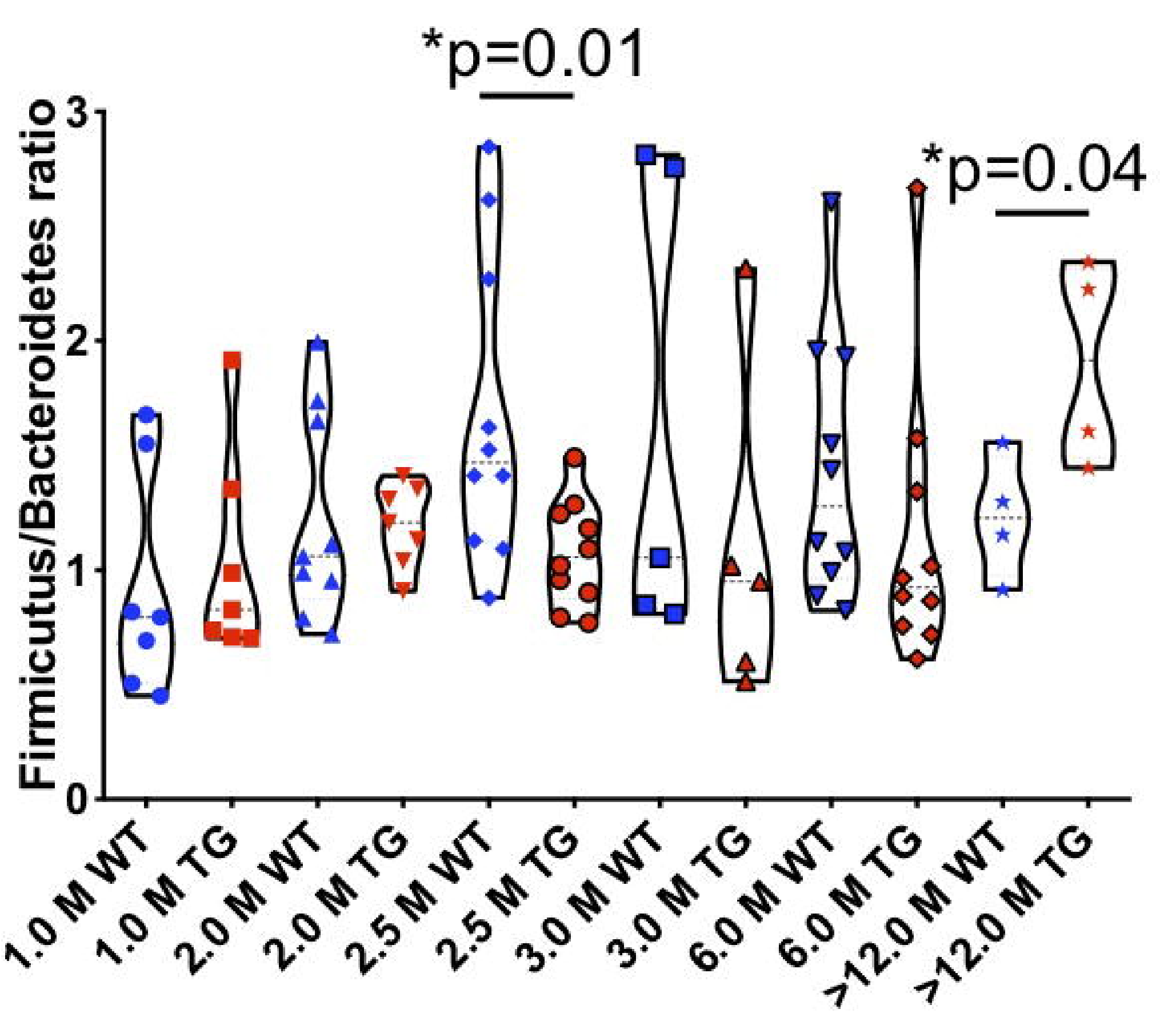

d

Time point (age in months)

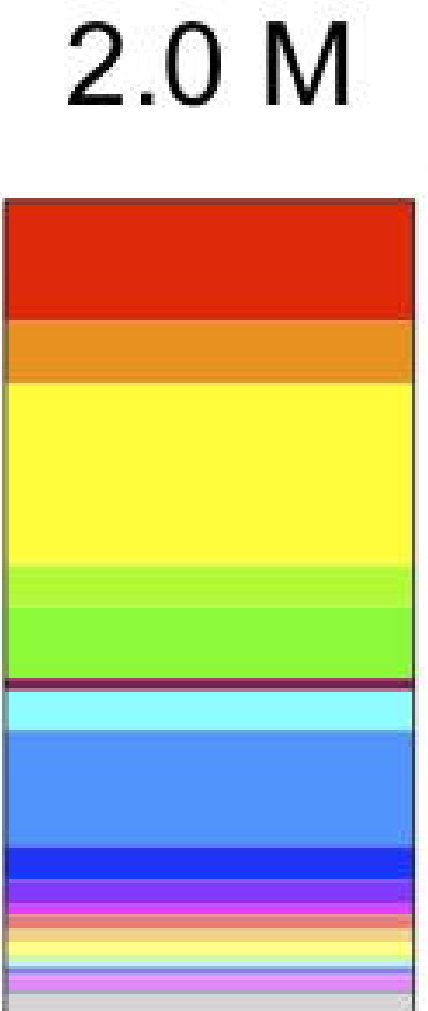

TG
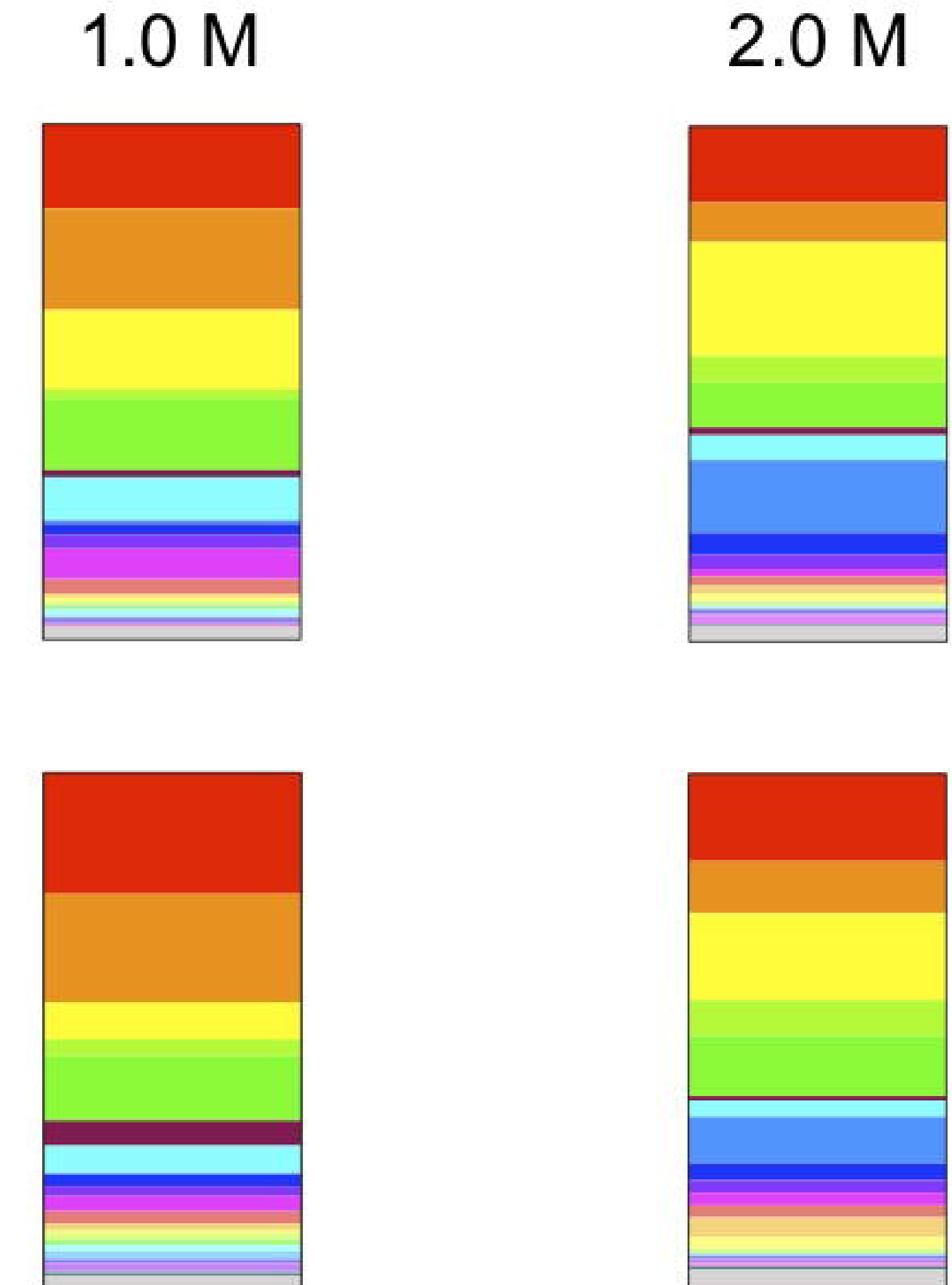

Lactobacillus $\left({ }^{* *} p=0.009\right)$ araprevotellatantramem $\left.{ }^{* *} \mathrm{p}=0.006\right)$

Oscillospira

$\left({ }^{*} \mathrm{p}=0.05\right)$

Paraprevotella $(p=0.057)$

$\left({ }^{*} \mathrm{p}=0.03\right)$

Turicibacter

( $\left.{ }^{*} \mathrm{p}=0.02\right)$

Marvinbryantia

$\left({ }^{* *} p=0.008\right)$

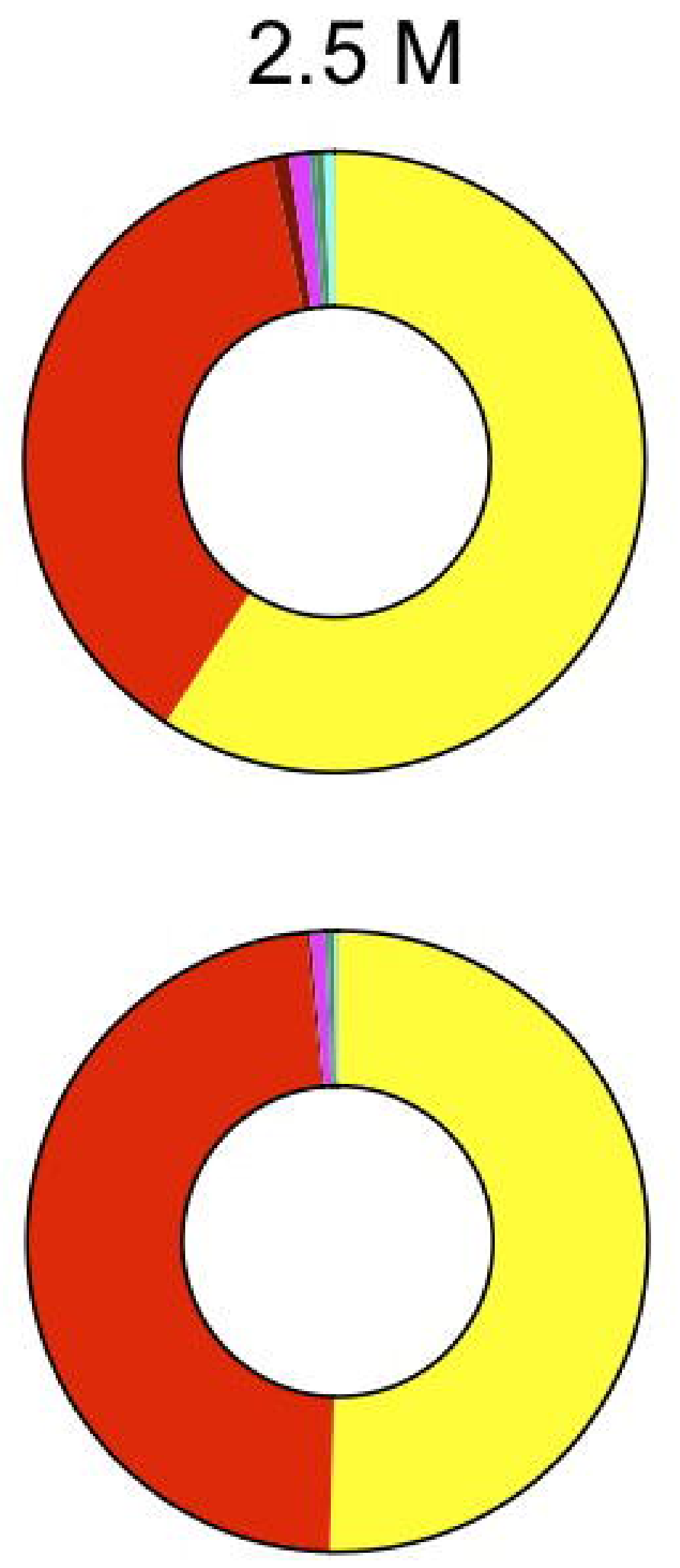

Firmicutes $\left({ }^{*} \mathrm{p}=0.02\right)$

Bacteroidetes $\left({ }^{* *} p=0.005\right)$

Actinobacteria $\left({ }^{*} p=0.04\right)$

Cyanobacteria * $\mathrm{p}=0.03$ )

Two-way ANOVA

Fixed effects (type III)

Time $=\mathrm{ns}$

Genotype $=$ ns

Time $\mathrm{x}$ genotype $={ }^{*} \mathrm{p}=0.03$

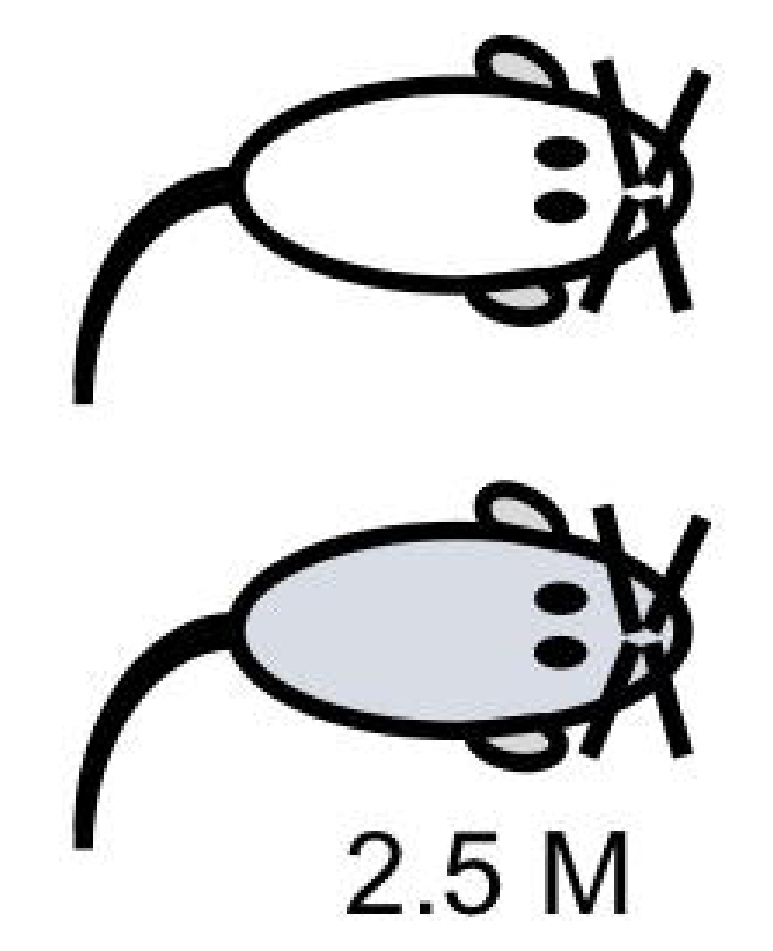

$3.0-6.0 \mathrm{M}$

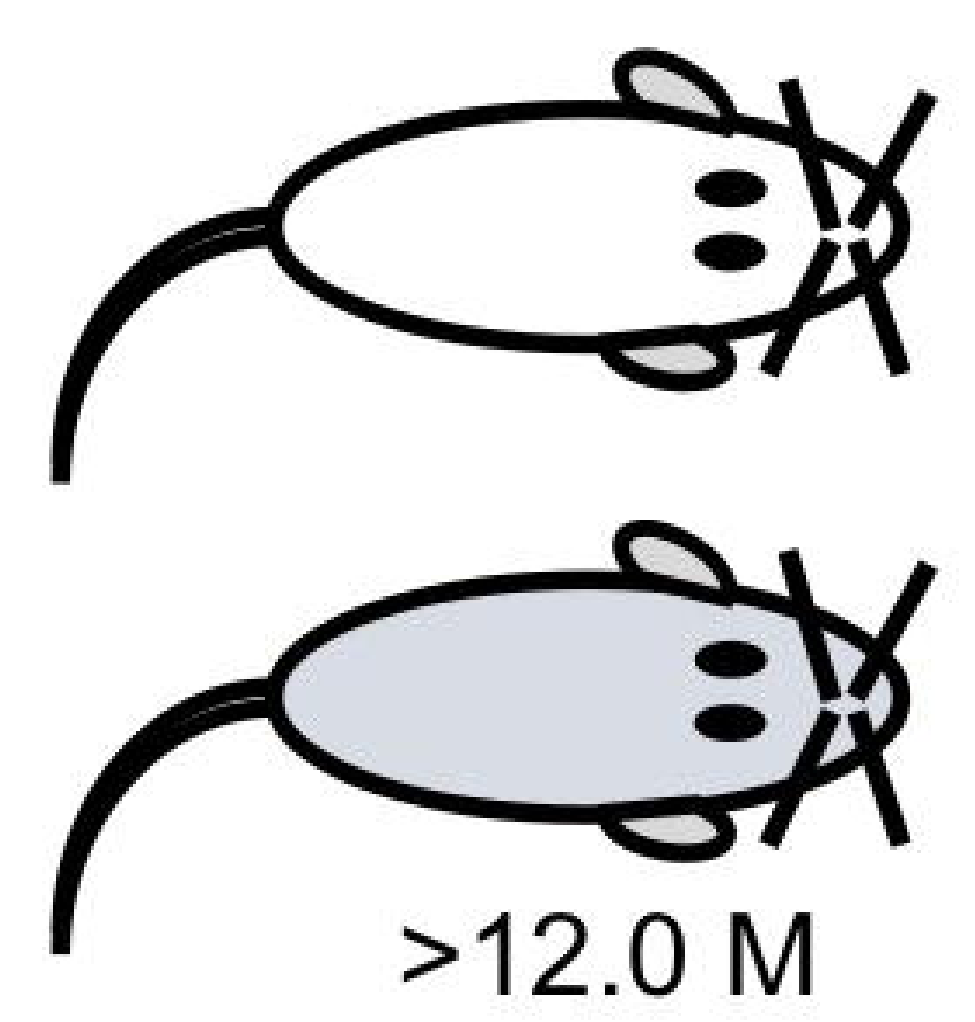

Homozygous SNCA-TG

유 \& \&
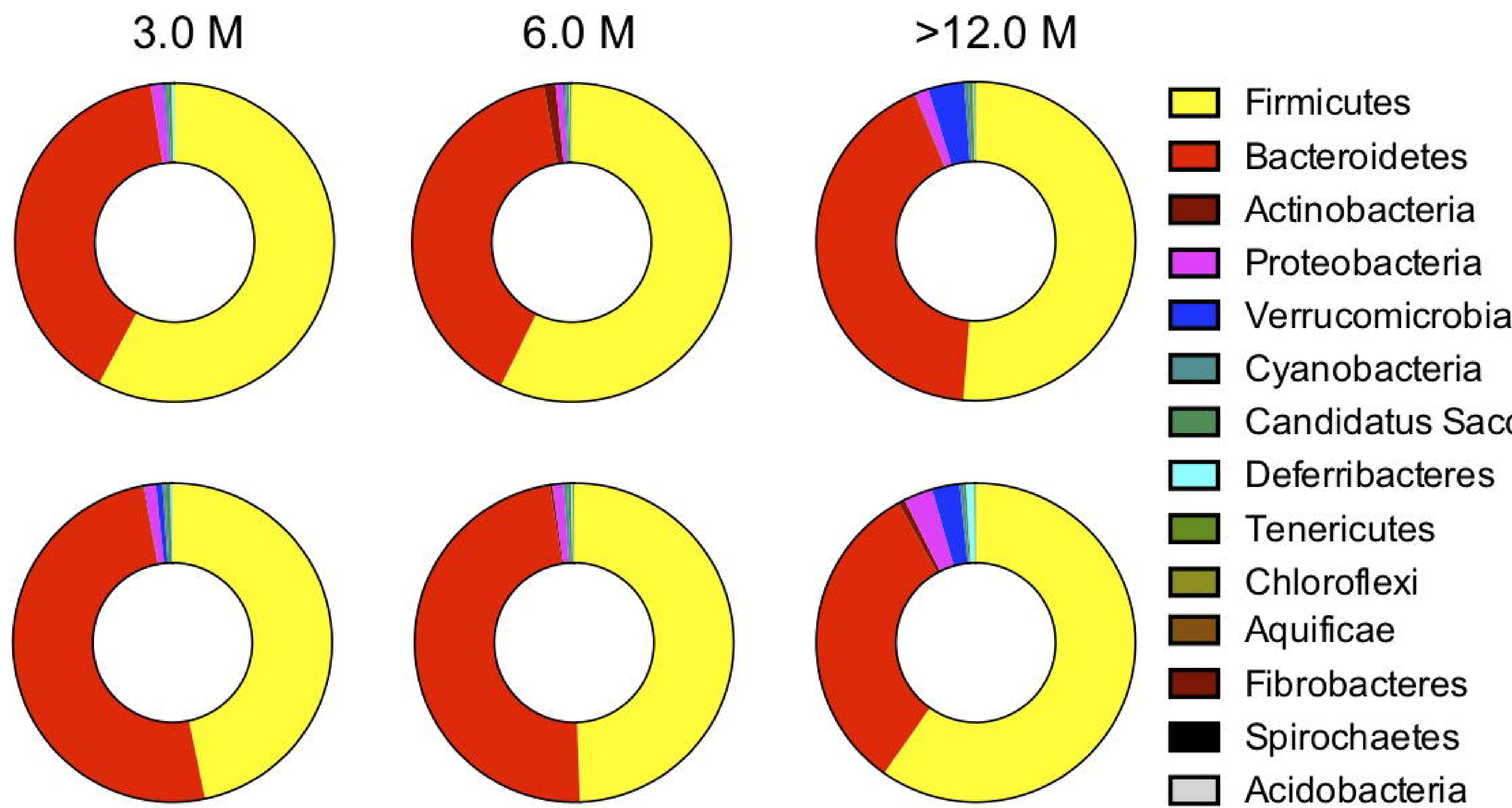

Actinobacteria

$\left({ }^{*} \mathrm{p}=0.02\right)$

Verrucomicrobia

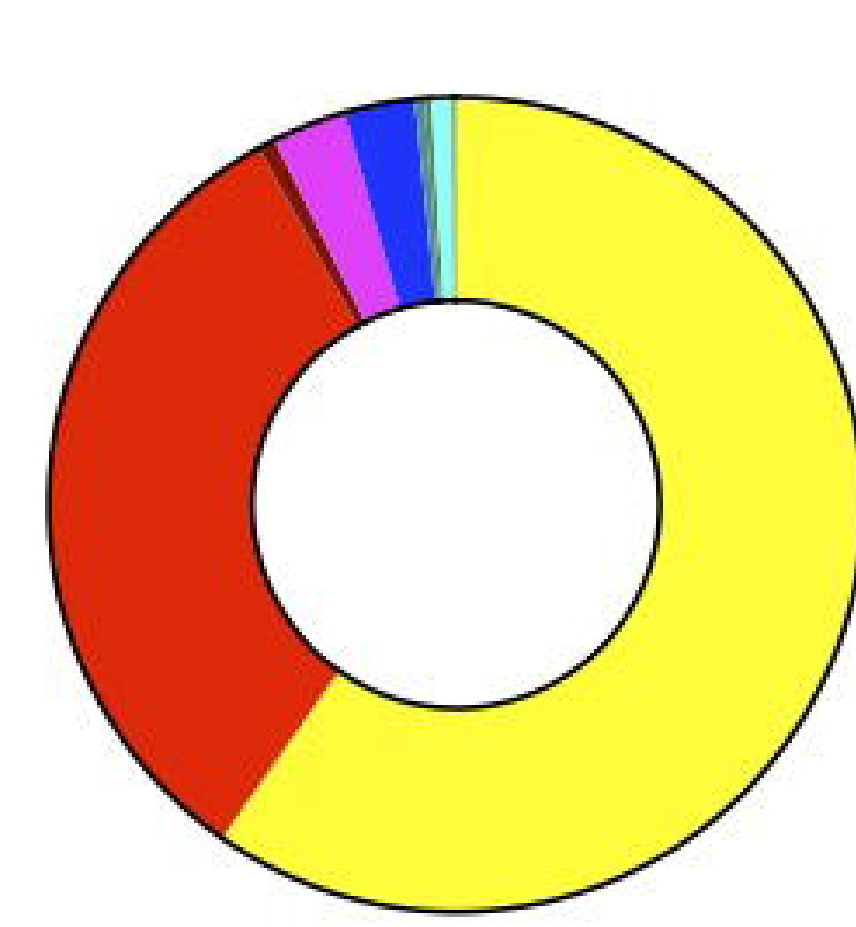

Cyanobacteria

$\square$ Candidatus Saccharibacteria

$\left({ }^{*} \mathrm{p}=0.01\right)$

Chloroflexi $\left({ }^{*} p=0.01\right)$

Bacteroidetes $\left({ }^{*} \mathrm{p}=0.02\right)$

Proteobacteria $(p=0.07)$

$\square$ Deferribacteres

$\square$ Tenericutes

$\square$ Chloroflexi

$\square$ Aquificae

$\square$ Fibrobacteres

- Spirochaetes $\square$ Acidobacteria
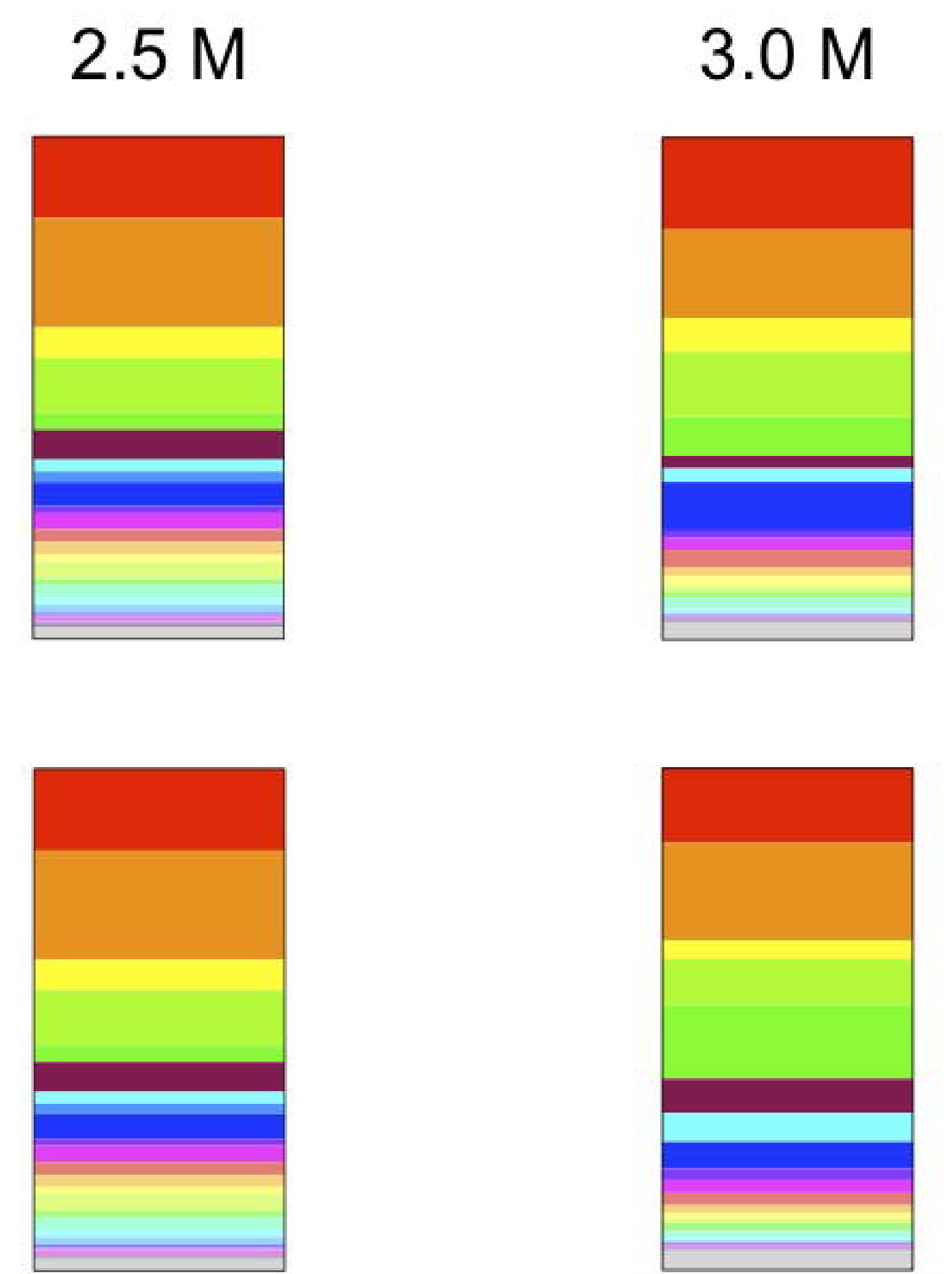

Prevotella

$$
\left({ }^{* * *} \mathrm{p}=0.0001\right)
$$

Ruminiclostridium ( ${ }^{*} \mathrm{p}=0.04$ )

Parabacteroides $\left({ }^{* *} p=0.006\right)$

Bifidobacterium ( ${ }^{*} \mathrm{p}=0.03$ )

Lachnoanaerobaculum $\left({ }^{*} \mathrm{p}=0.01\right)$

Lachnospira $\left({ }^{*} \mathrm{p}=0.02\right)$

Turicibacter $\left({ }^{*} \mathrm{p}=0.02\right)$

Oscillospira ${ }^{* *}(p=0.008)$

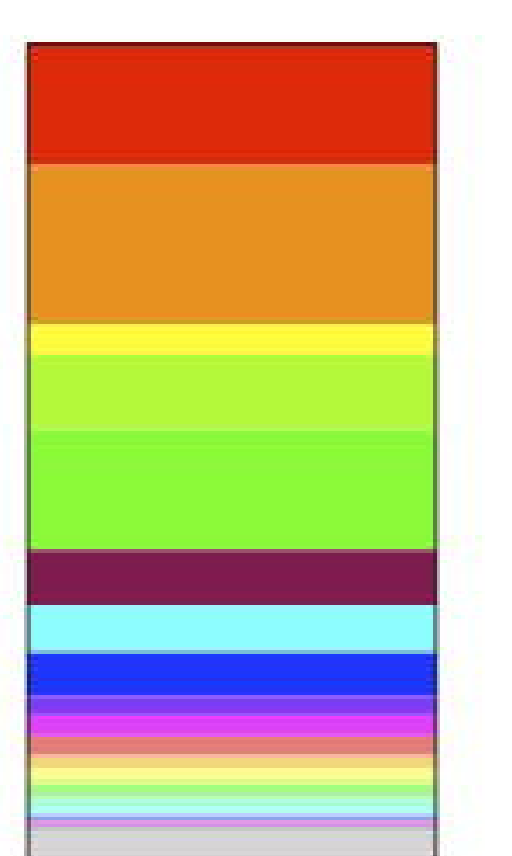

Alistipes $\left({ }^{*} \mathrm{p}=0.03\right)$ Alloprevotella

$\left.{ }^{* *} p=0.008\right)$

Lactobacillus

$\left({ }^{* *} p=0.002\right)$

Bifidobacterium

( $\left.{ }^{*} p=0.02\right)$

Turicibacter

$\left({ }^{* * *} \mathrm{p}=0.0006\right)$
$>12.0 \mathrm{M}$
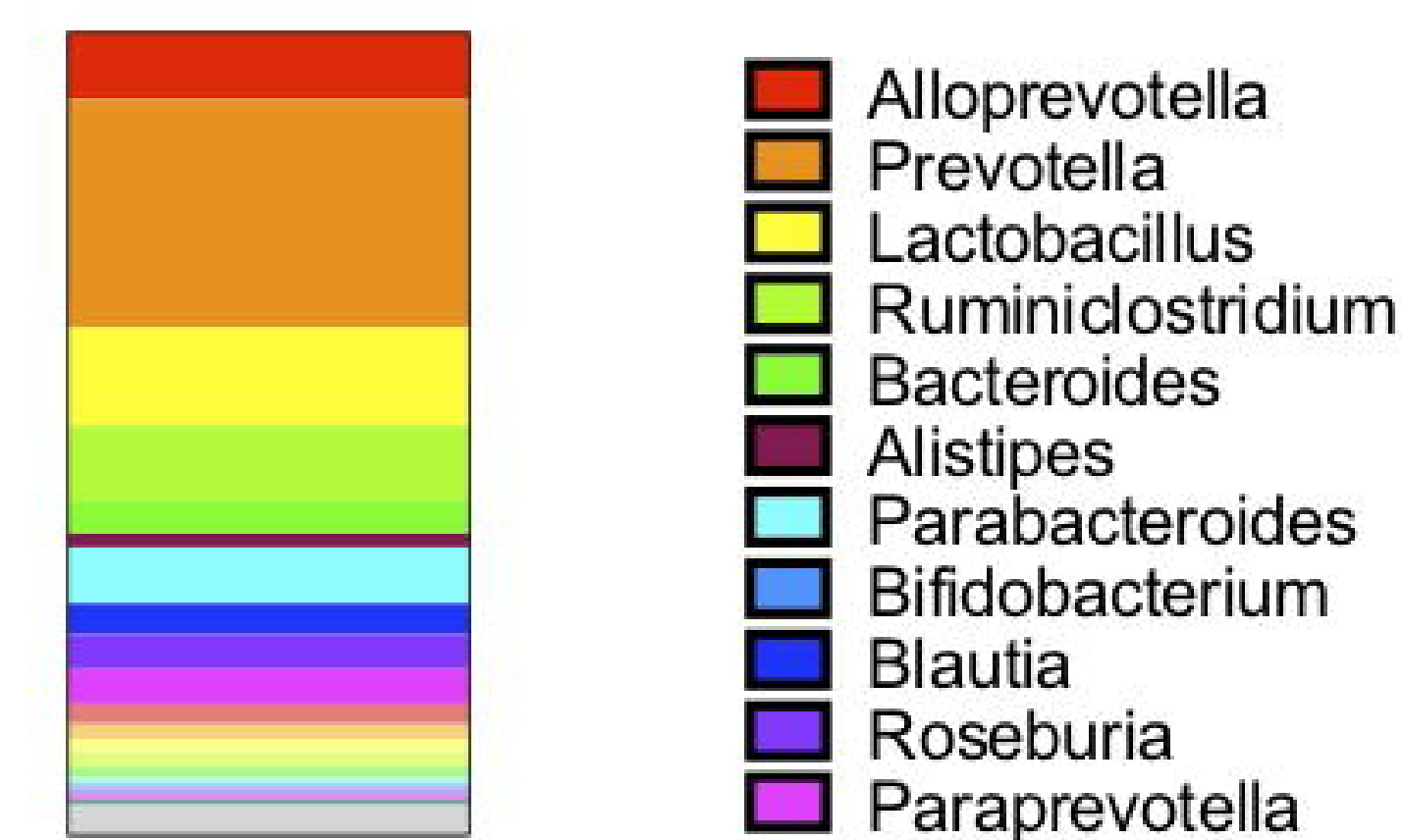

Bacteroides

Parabacteroides

Bifidobacterium

Blautia

Roseburia
Paraprevotella
Streptococcus

Anaerotruncus

Desulfovibrio

Ruminococcus

Lachnoanaerobaculum

Lachnospira

Barnesiella

Barnesiella

Turicibacter
Marvinbryantia Marvinbryantia
Oscillospira

Alloprevotella $\left({ }^{*} p=0.03\right)$

Prevotella $\left({ }^{* * *} p=0.0001\right)$

Lactobacillus ( $\left.{ }^{*} \mathrm{p}=0.01\right)$

Alistipes $\left({ }^{* * *} p=0.0005\right)$

Parabacteroides $\left({ }^{* *} \mathrm{p}=0.009\right)$

Anaerotruncus $\left({ }^{*} \mathrm{p}=0.04\right)$

Desulfovibrio ( $\left.{ }^{*} \mathrm{p}=0.01\right)$

Ruminococcus $\left({ }^{*} p=0.02\right)$

e

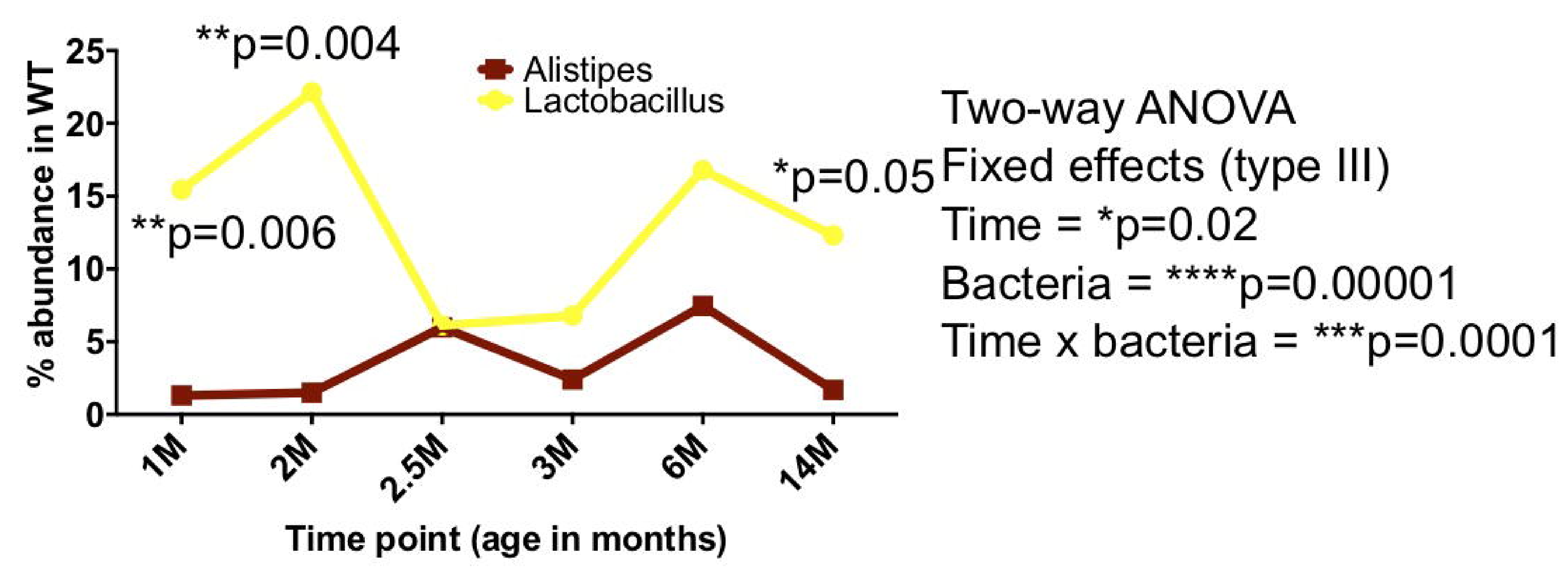

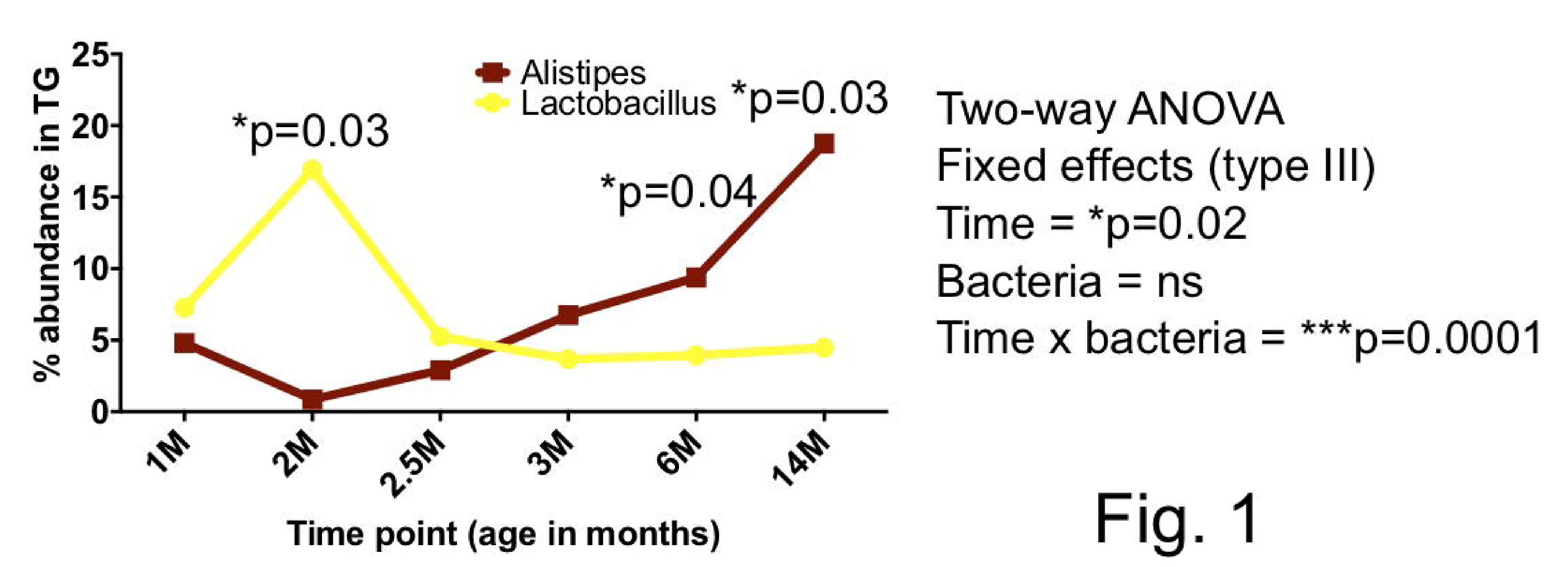


a
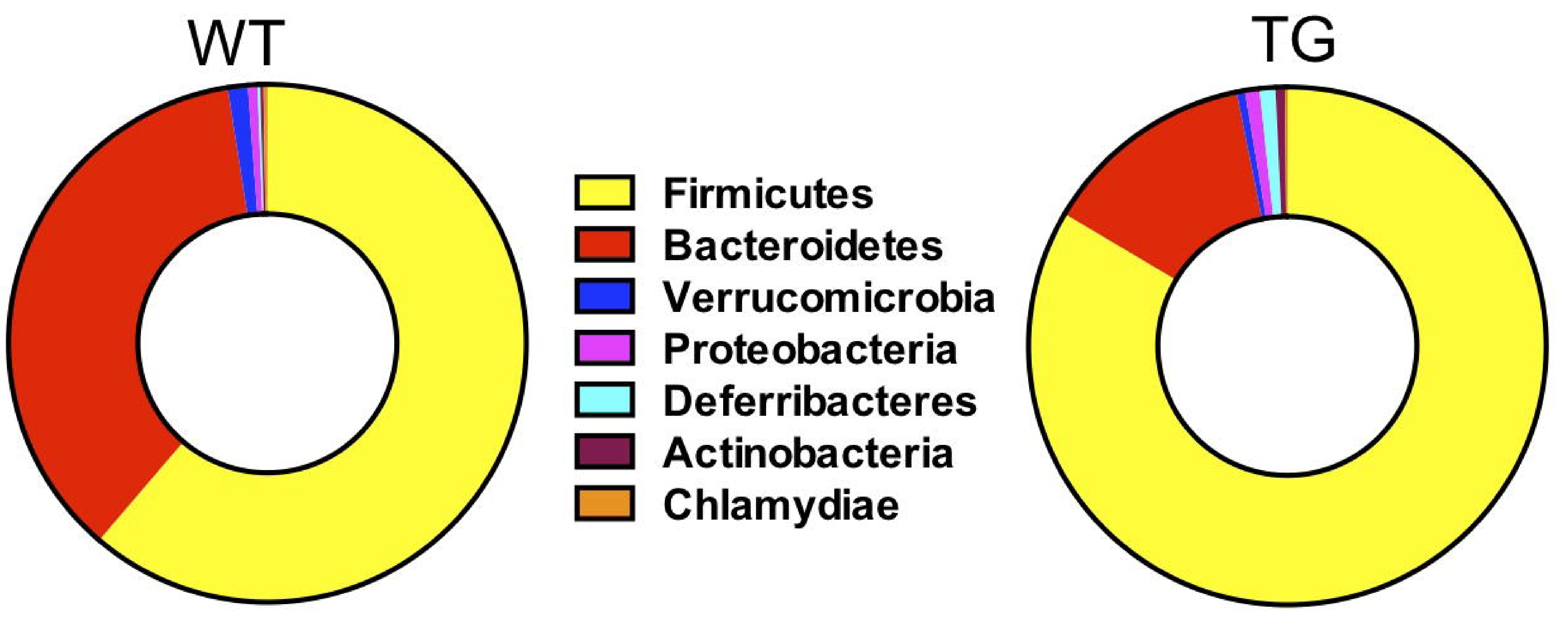

C WT

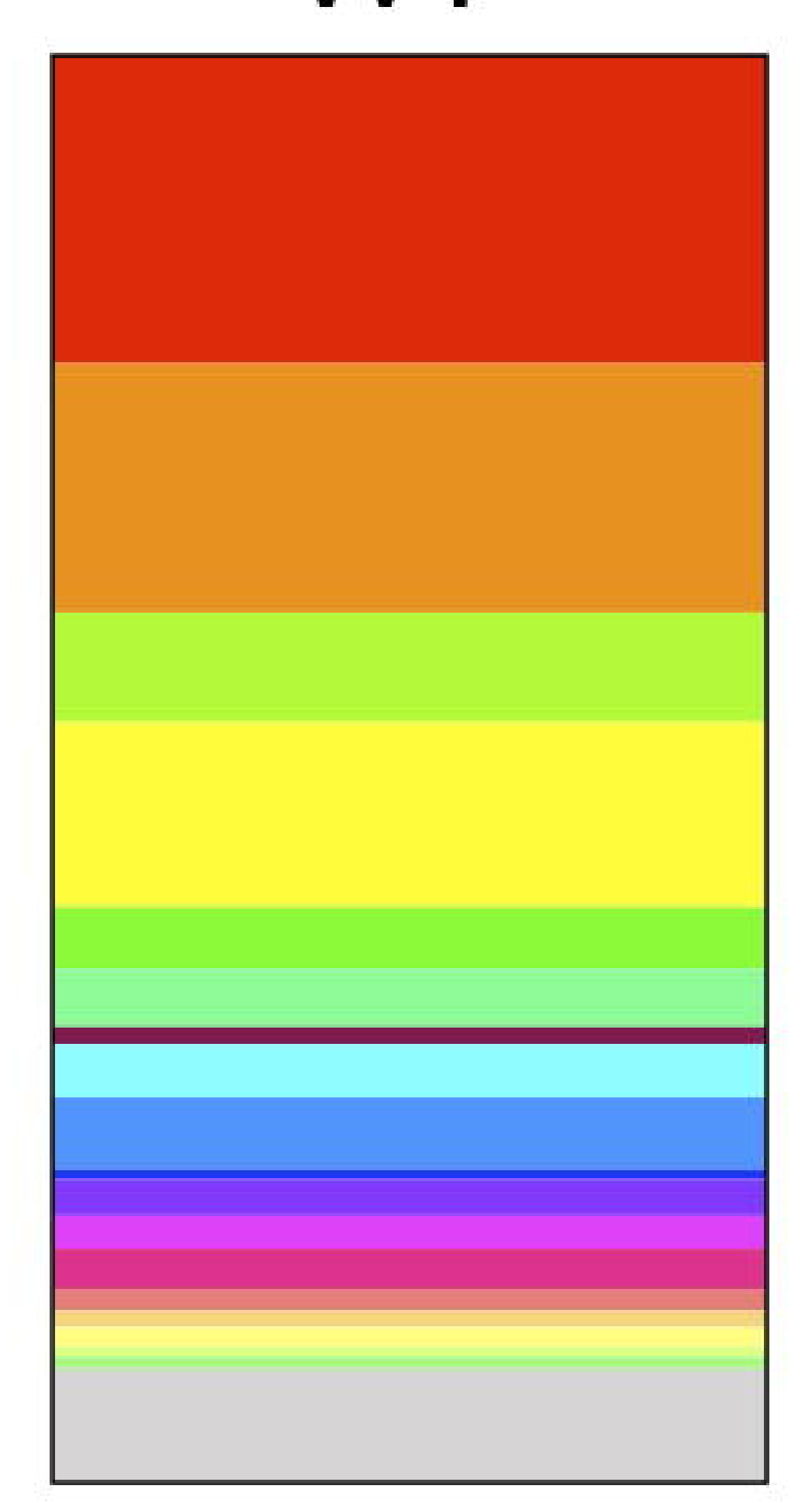

e

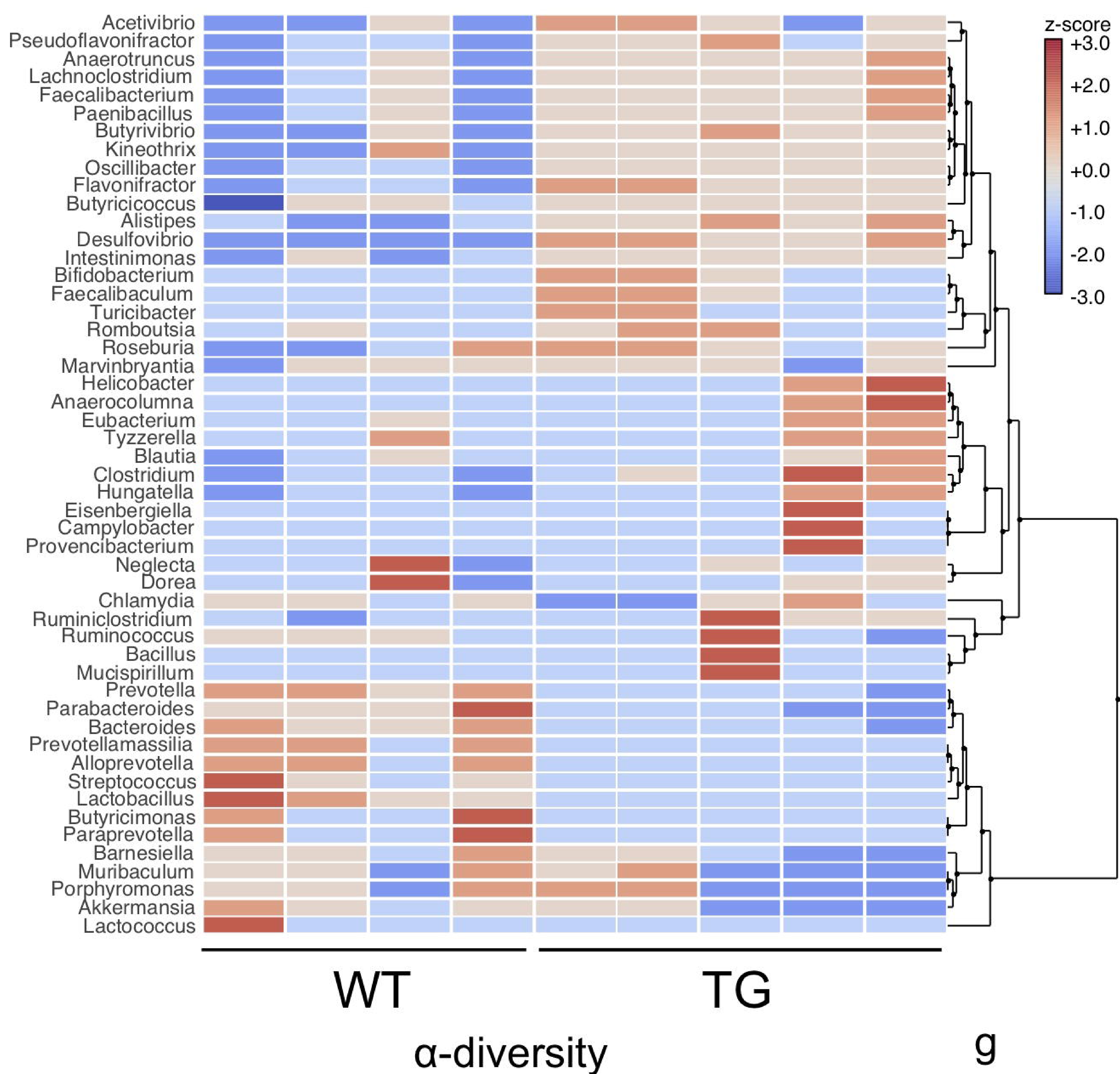

Firmicutes Bacteroidetes

Verrucomicrobia

Proteobacteria

Deferribacteres

Actinobacteria

Chlamydiae

- Spirochaetes

d

$\square$ Prevotella

Bacteroides

Eubacterium

Lactobacillus

Clostridium

Alistipes

Lachnoclostridium

Akkermansia

Mucispiril

Ruminococcus

Parabacteroides

Roseburia

므라ivibrio

므 Blautia

므 Flavonifractor

믈 Pseudoflavonifractor
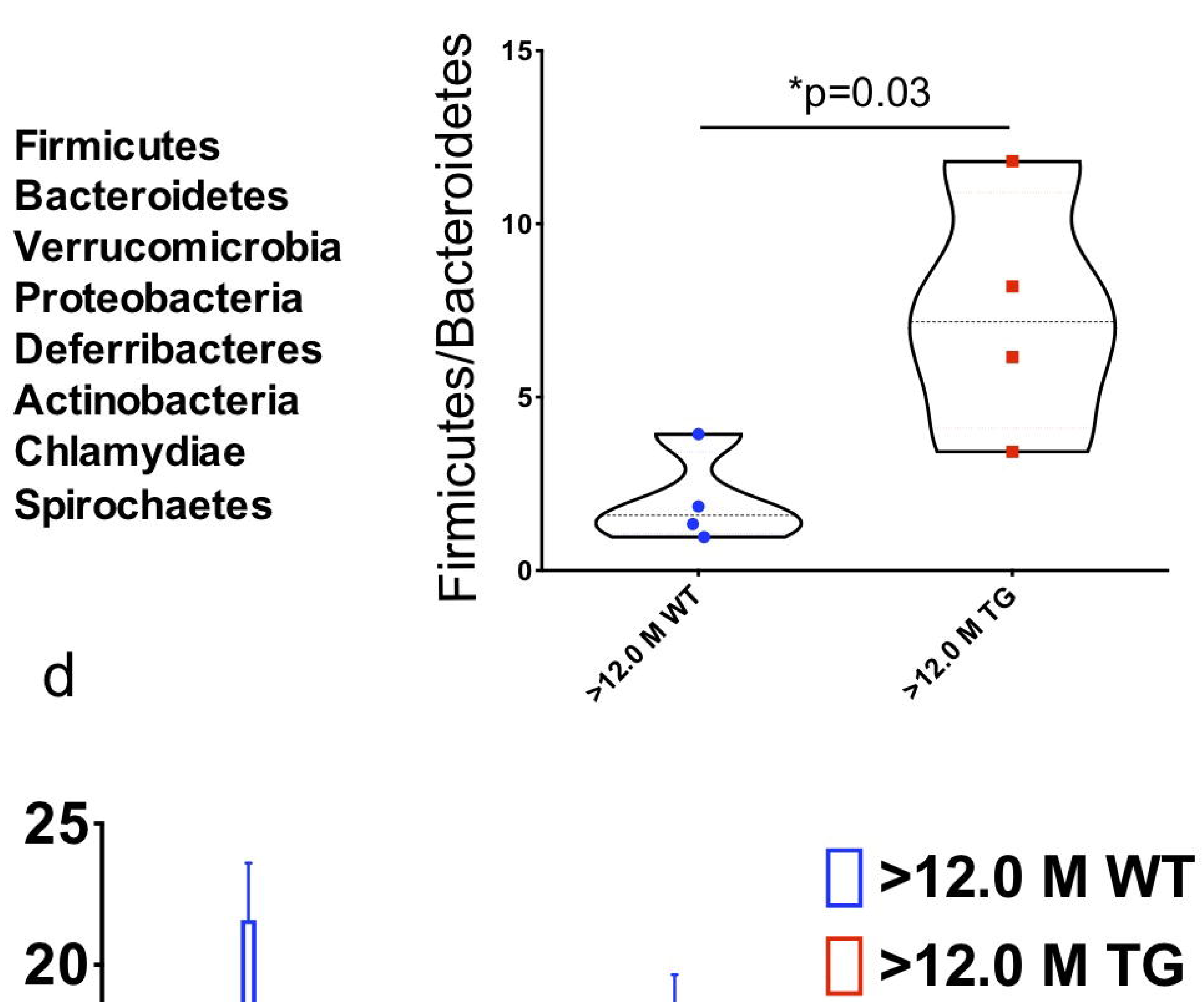
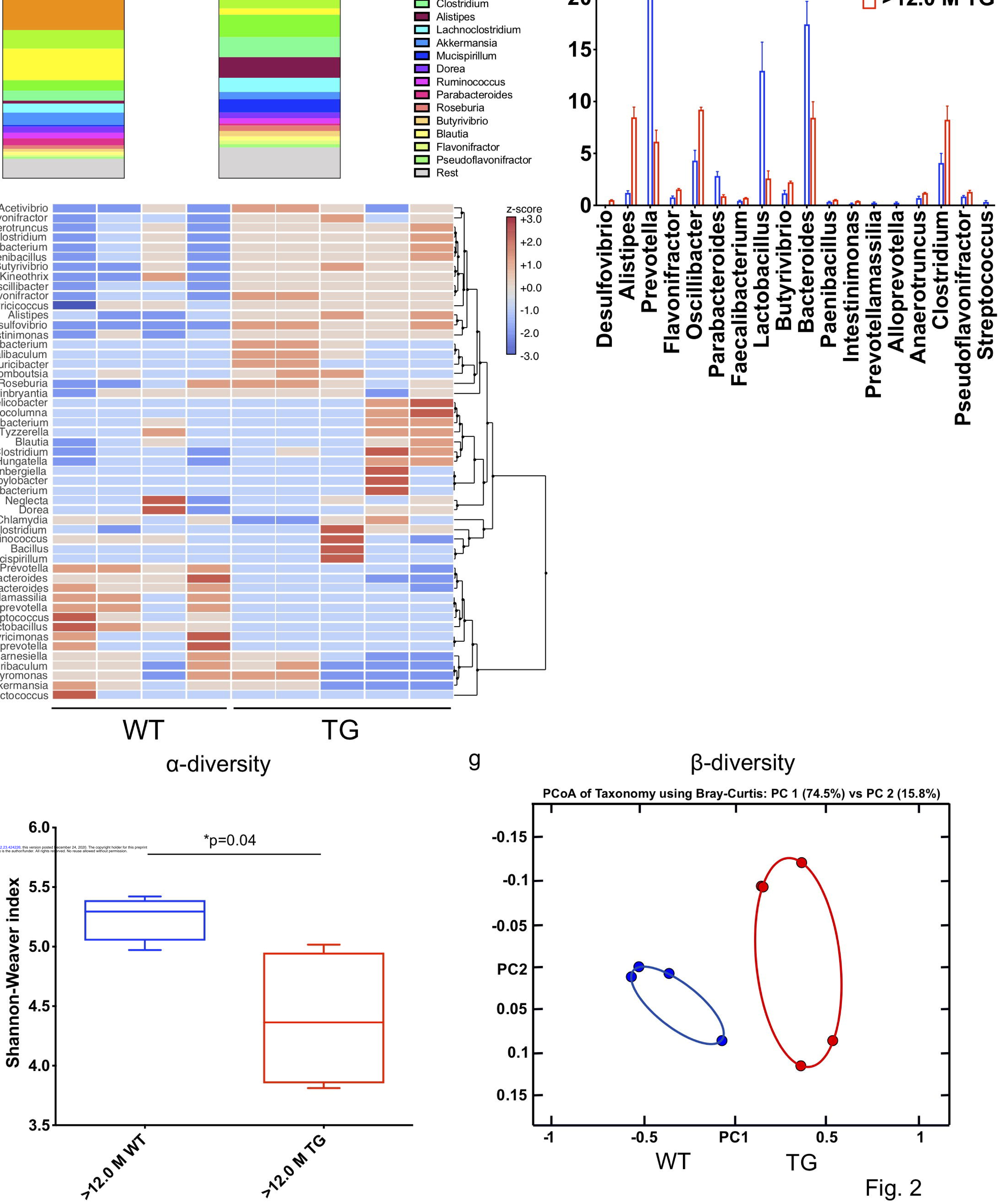

Fig. 2 
a

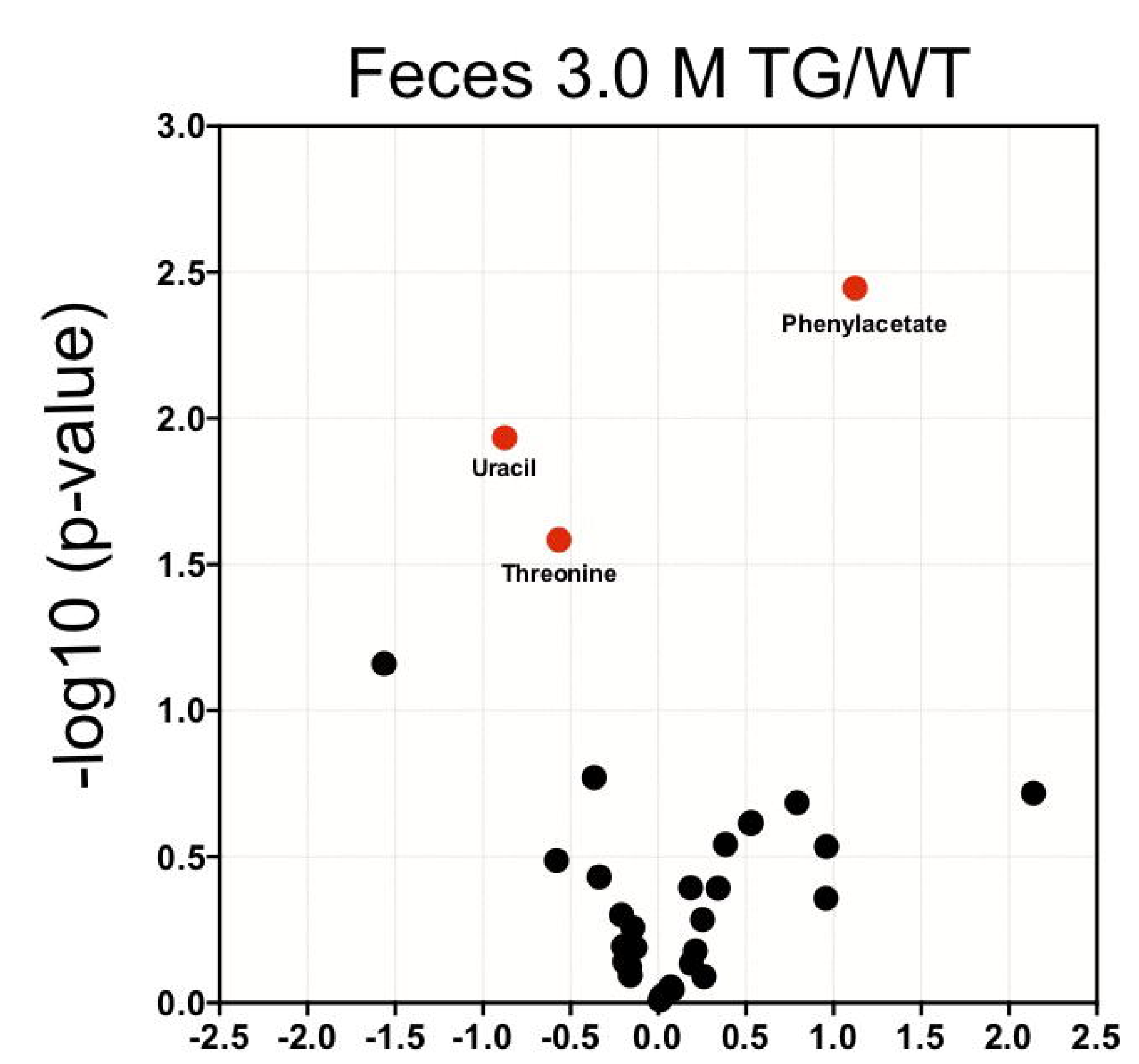

$\log 2(\mathrm{FC})$

b

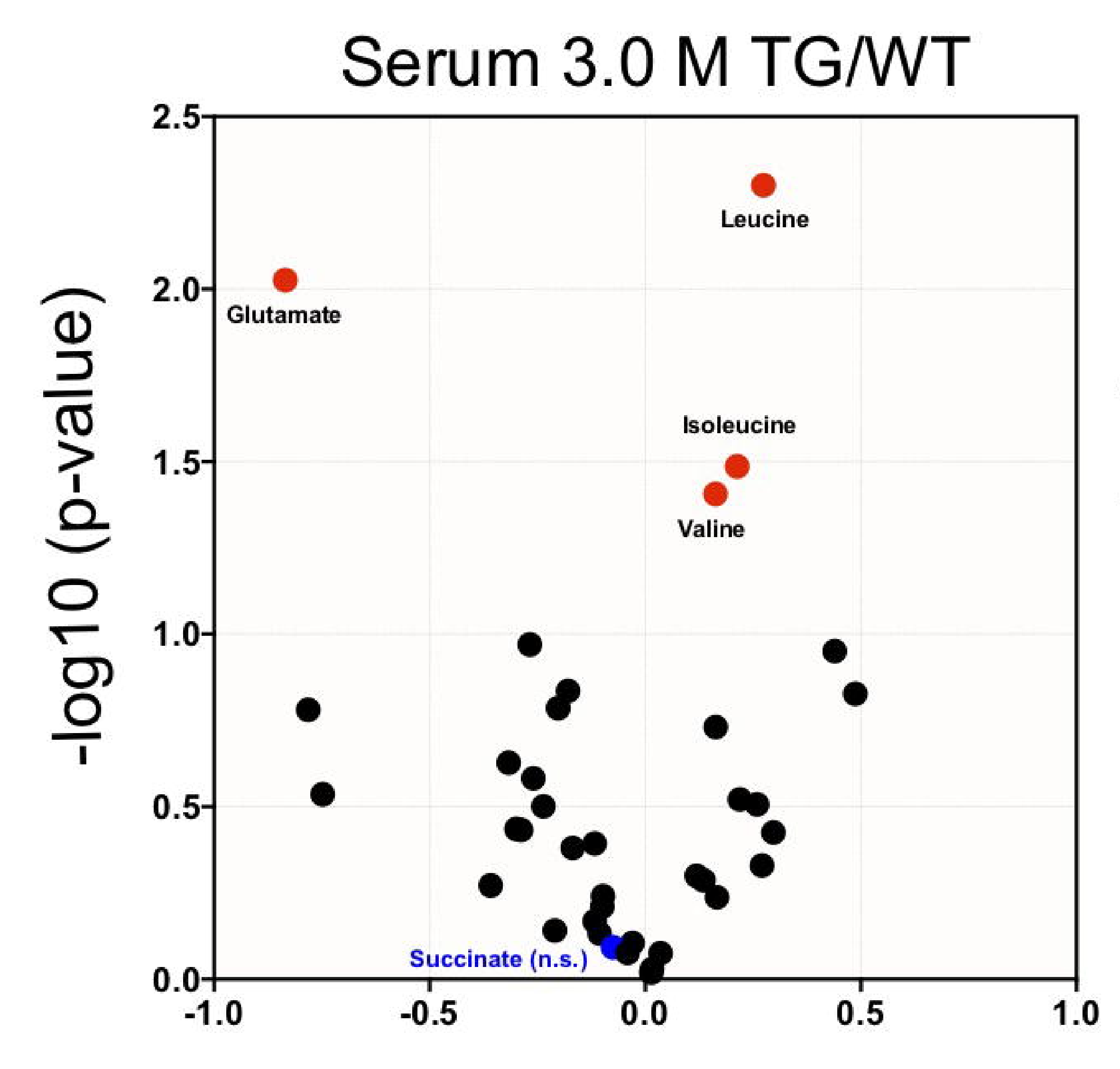

$\log 2(\mathrm{FC})$

C Serum $>12.0 \mathrm{M} \mathrm{TG} / 3.0 \mathrm{M}$ TG

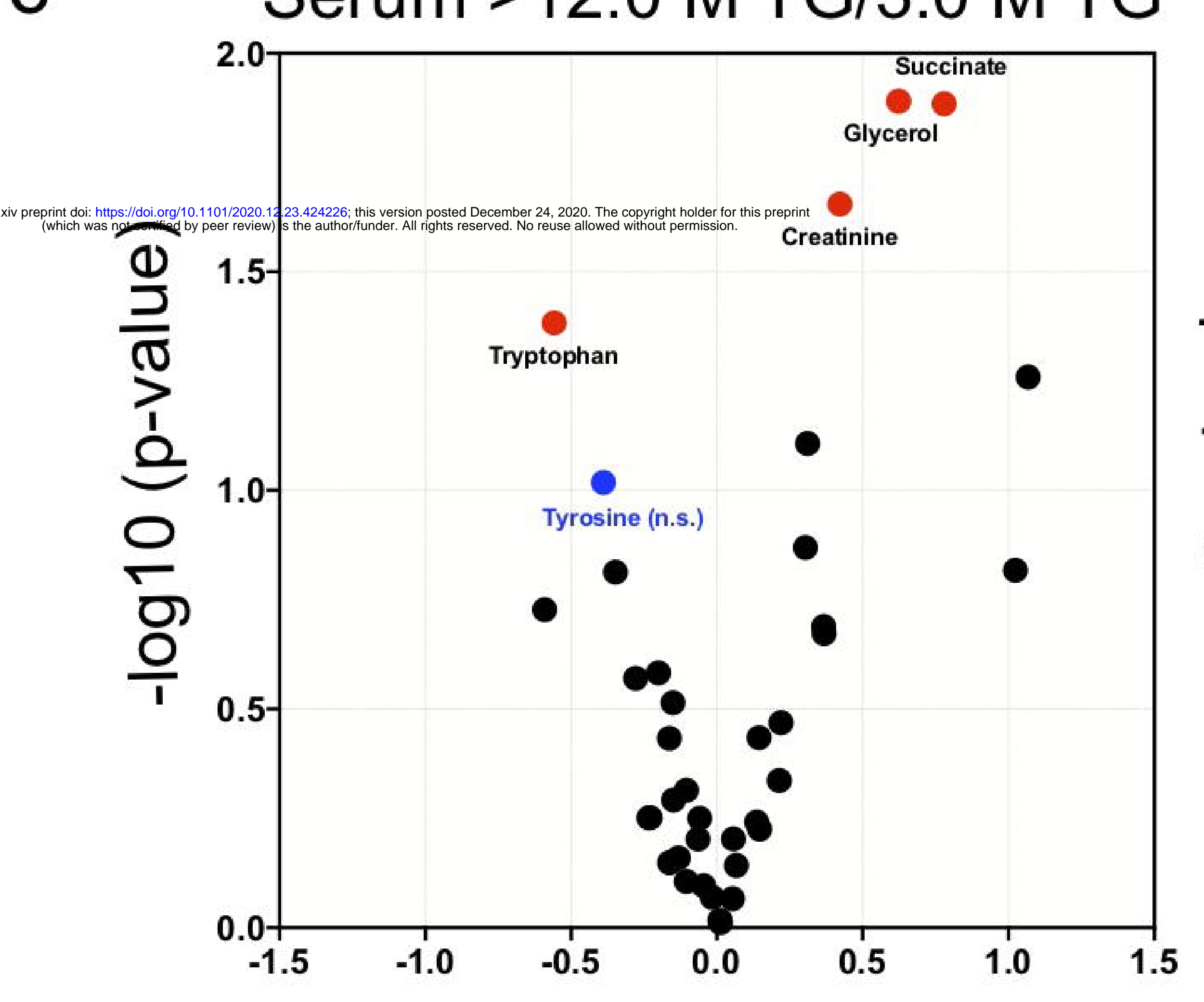

$\log 2(\mathrm{FC})$
Phenylacetate

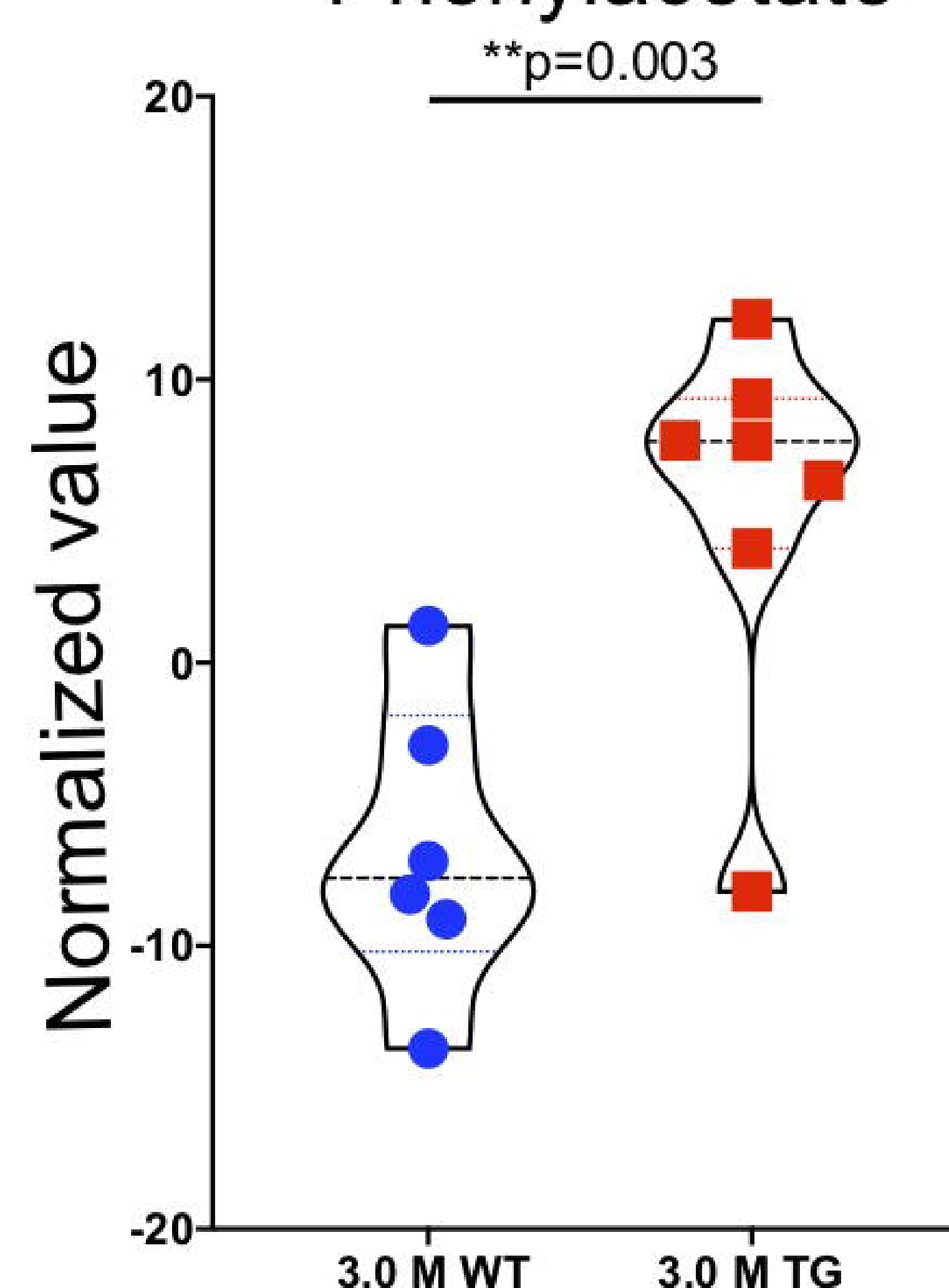

Glutamate

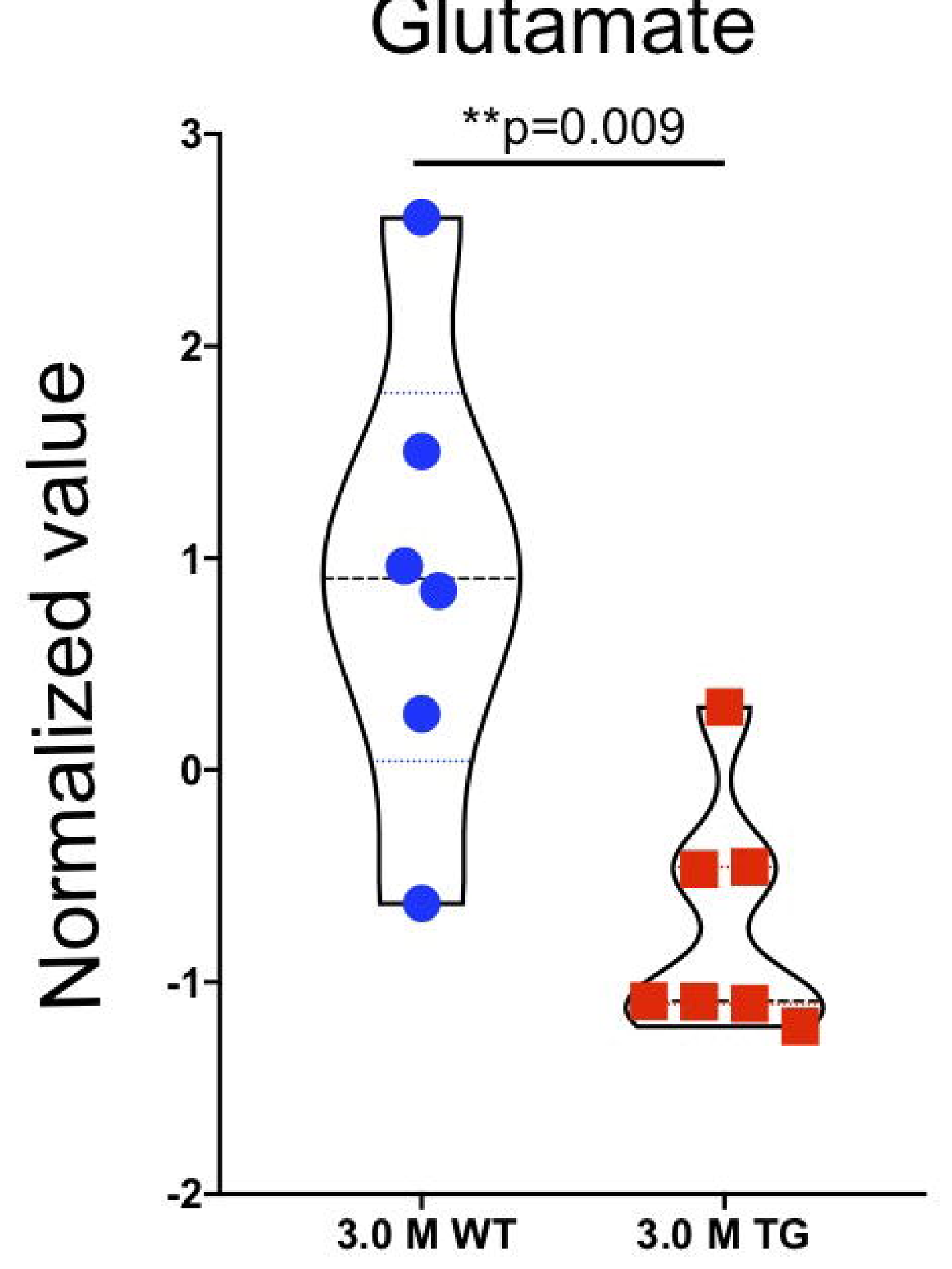

Succinate

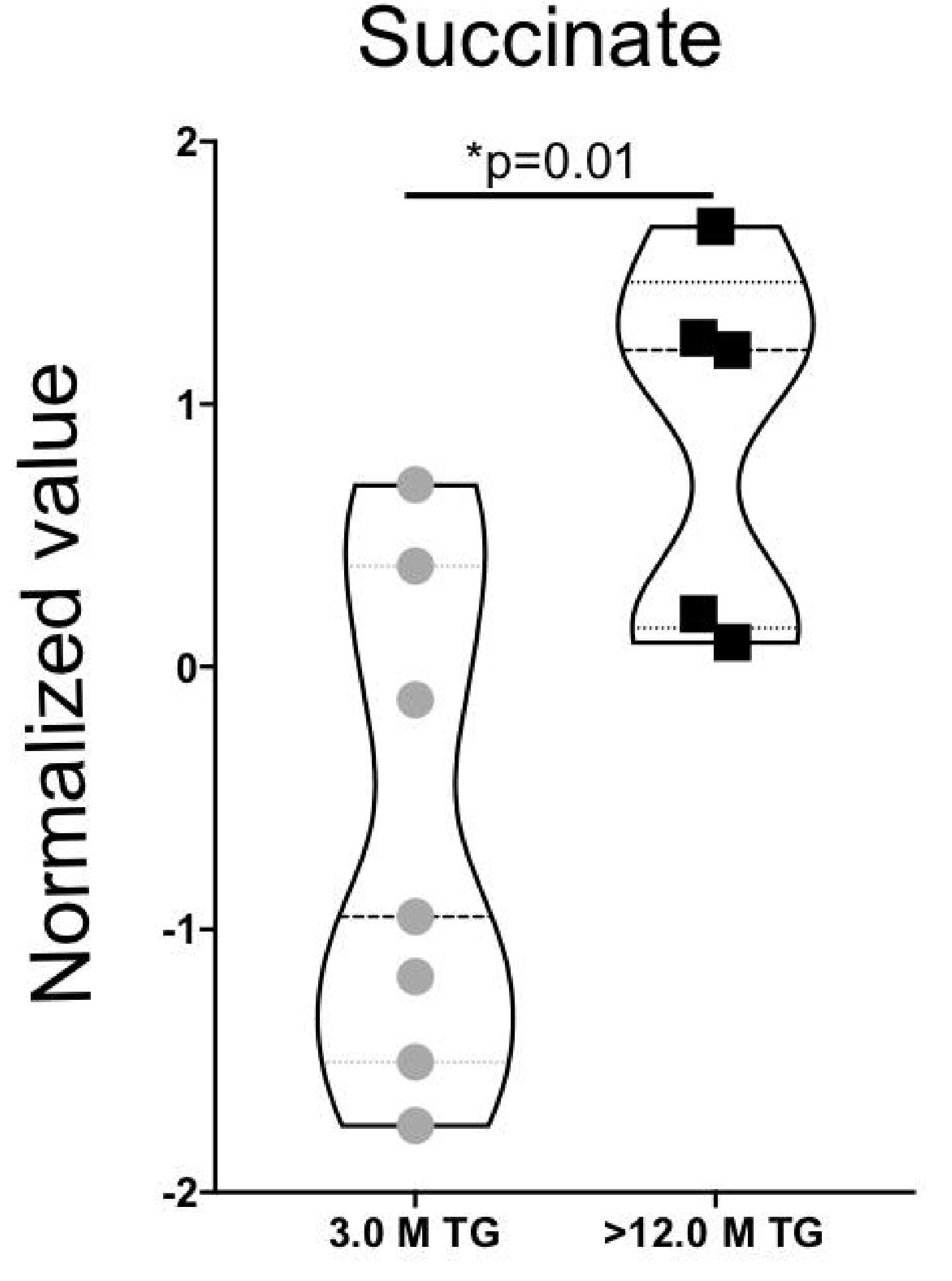

4-Hydroxyphenylacetate
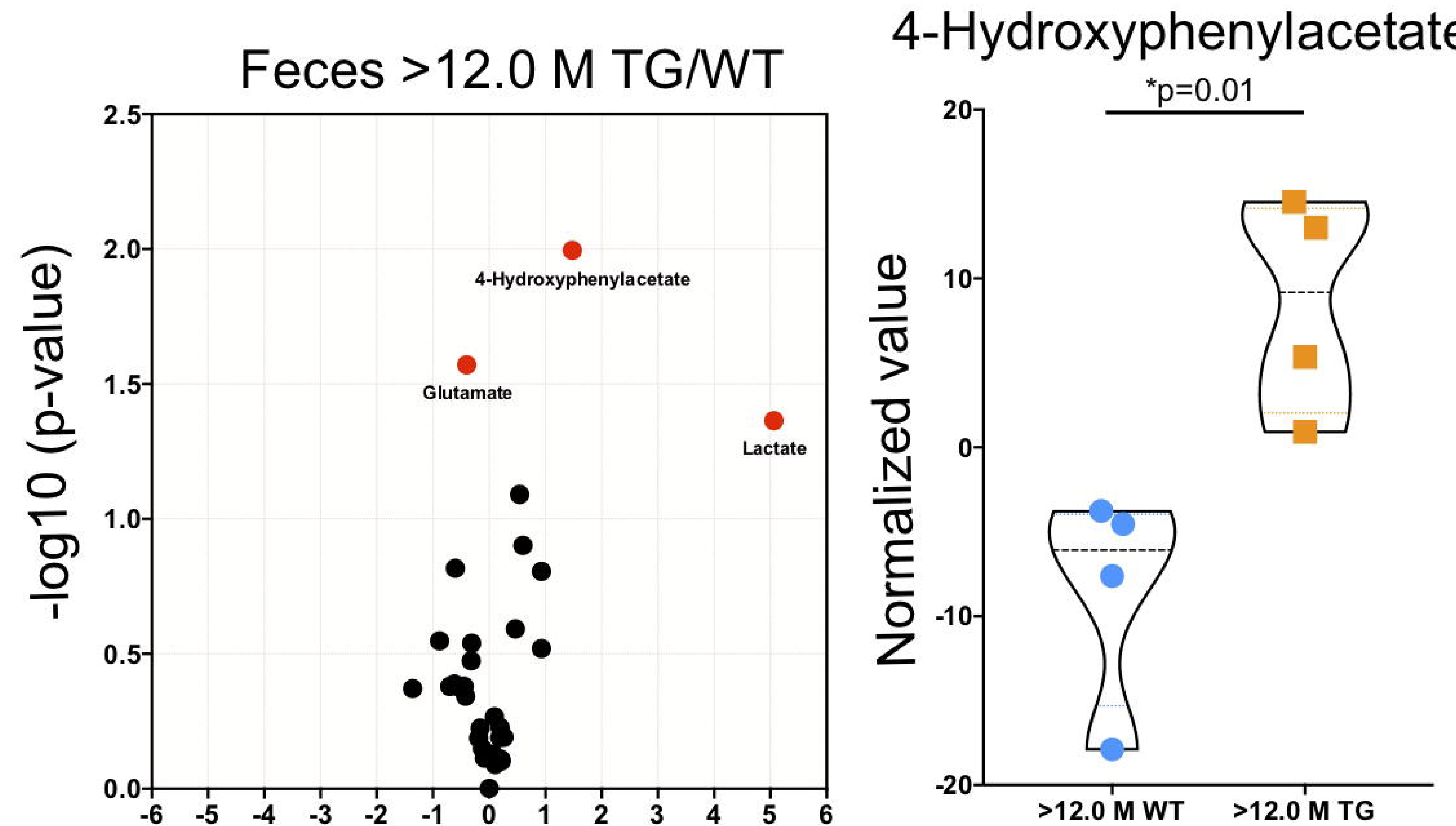

$\log 2$ (FC)

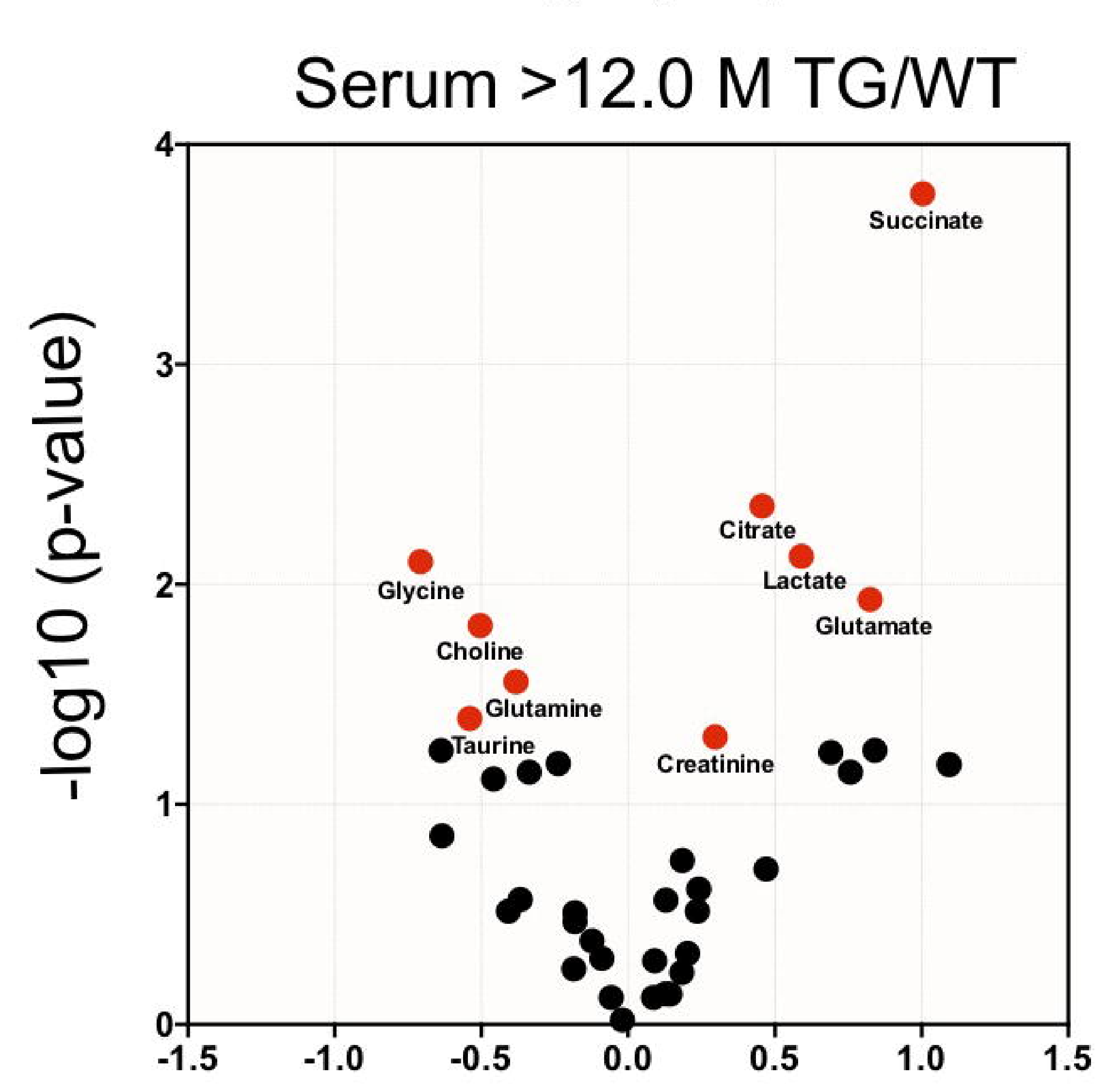

$\log 2(\mathrm{FC})$
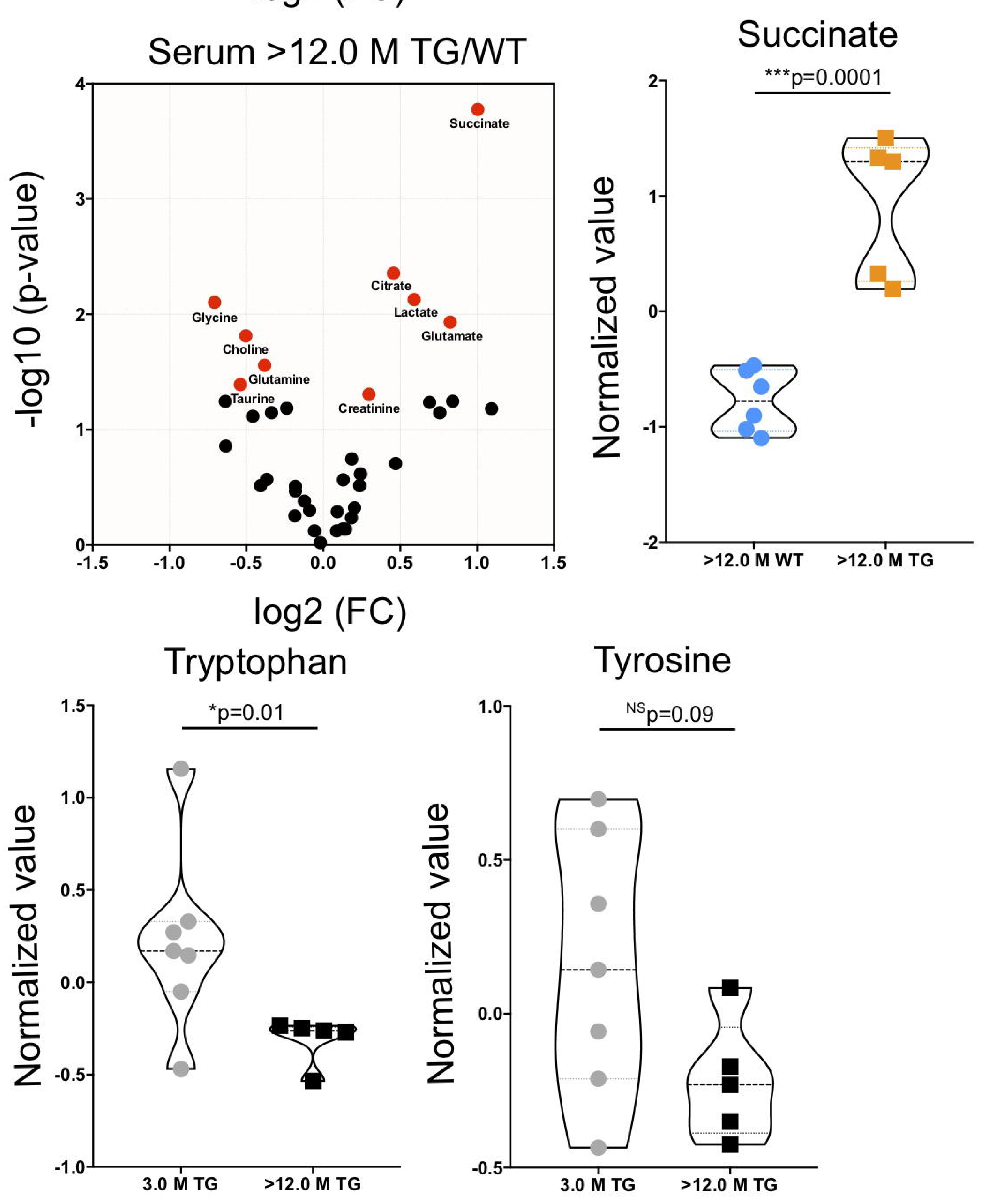
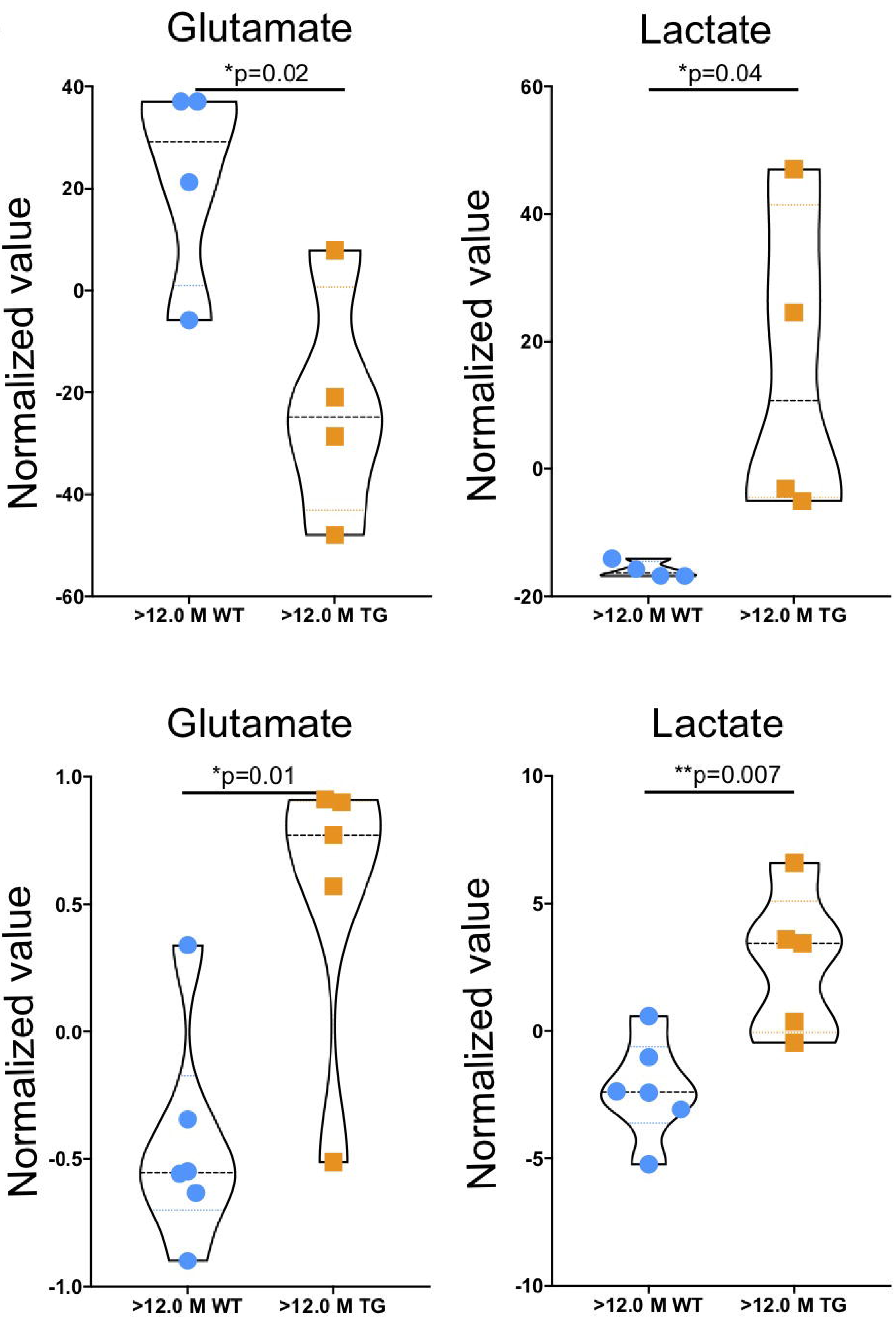

Top 25 compounds correlated with the Succinate

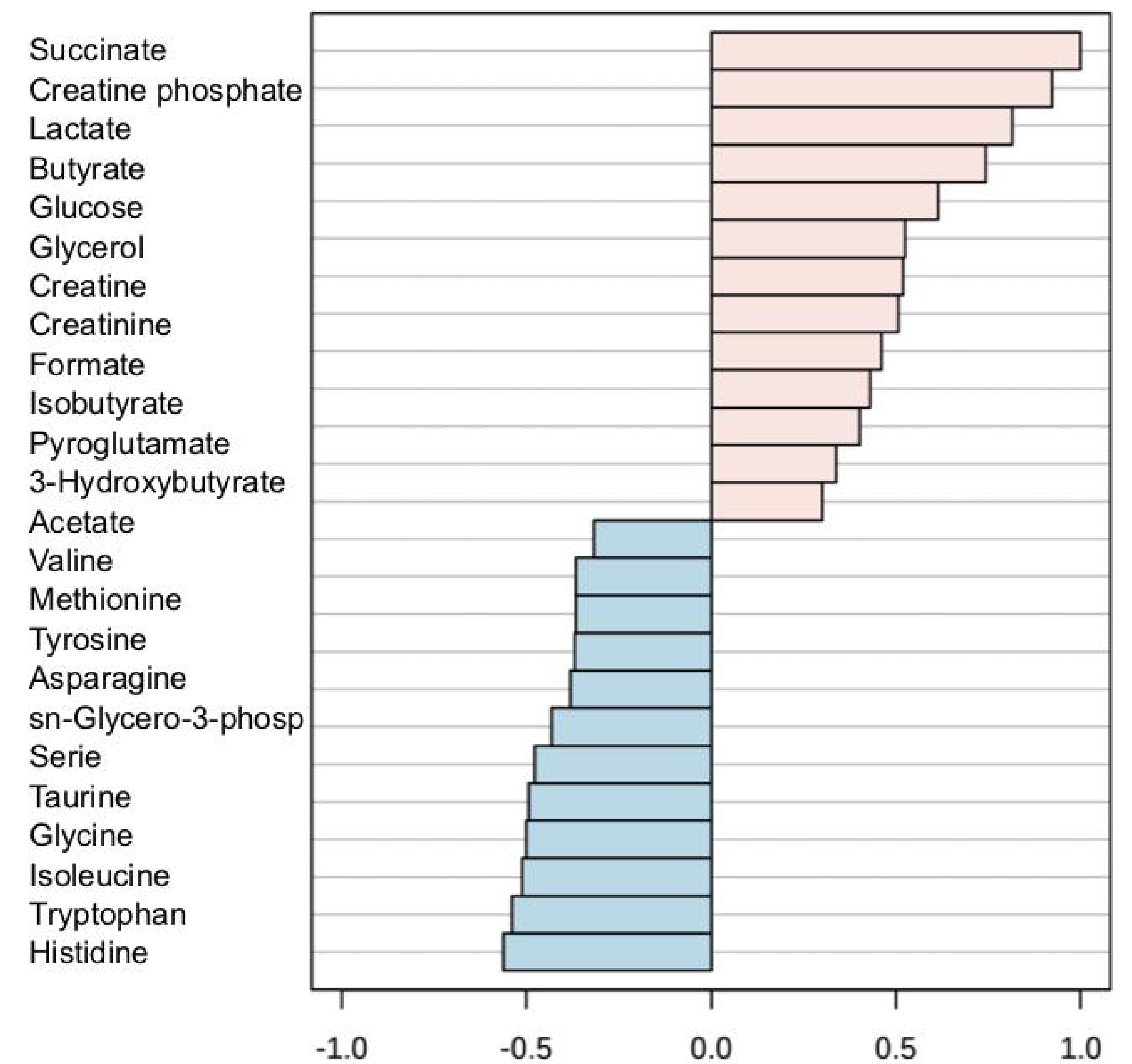

Correlation coefficient (Serum ageing effect: $3.0 \mathrm{M} \mathrm{TG}>>12.0 \mathrm{M} \mathrm{TG}$

Fig. 3 

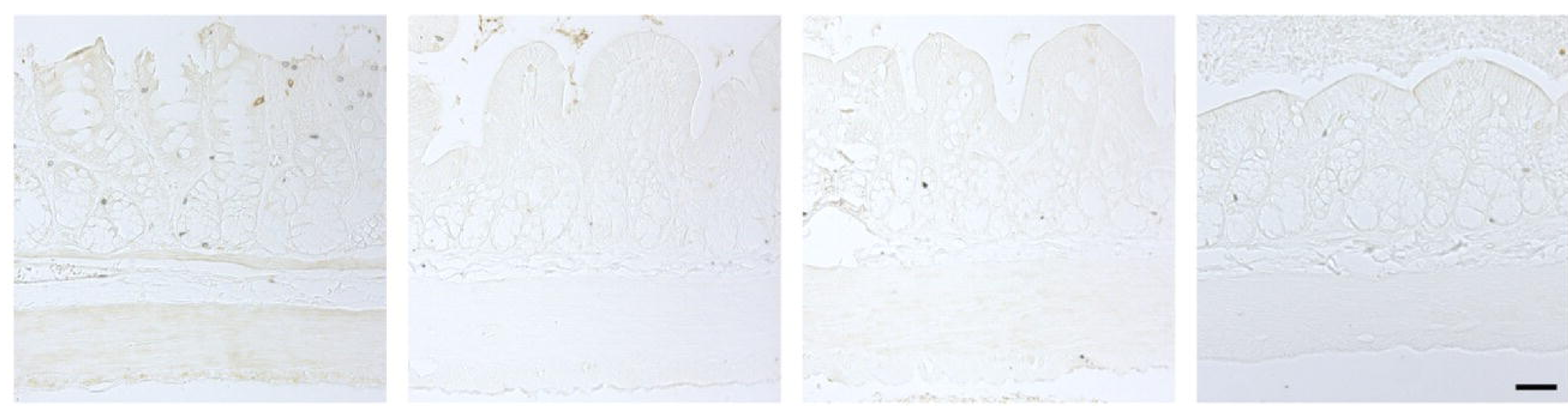

WT
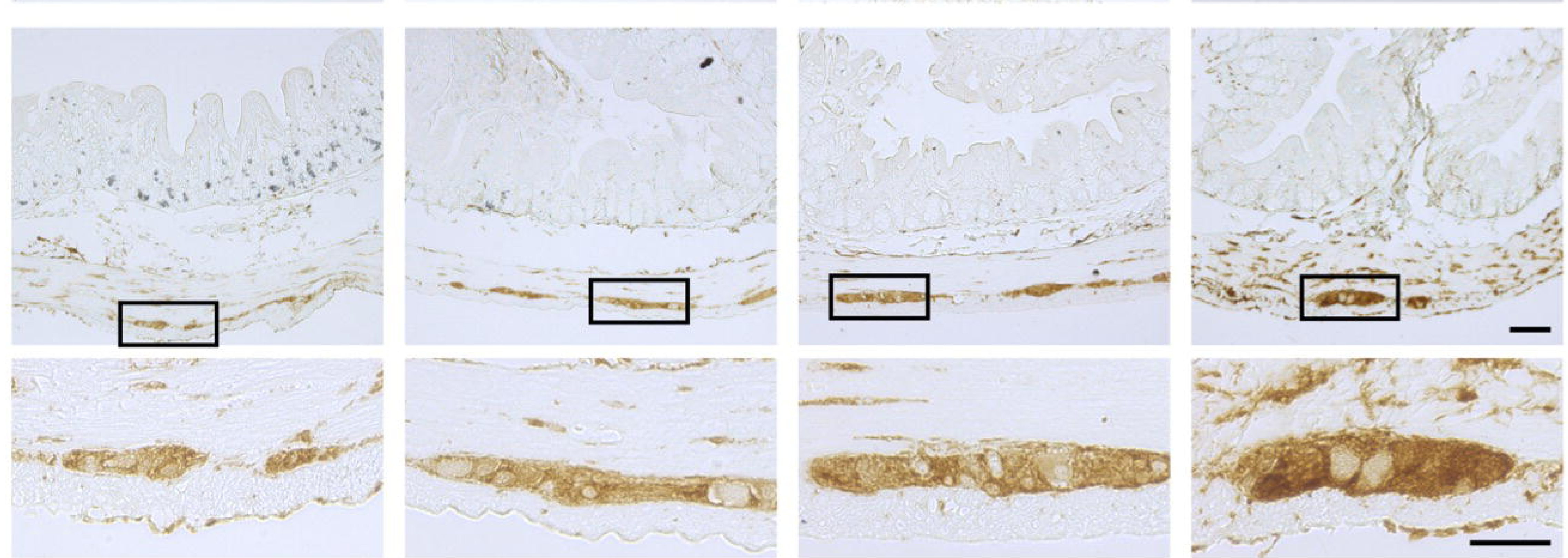

Human primary

total $\alpha$-syn antibody

(\#ab27766) LB509

b
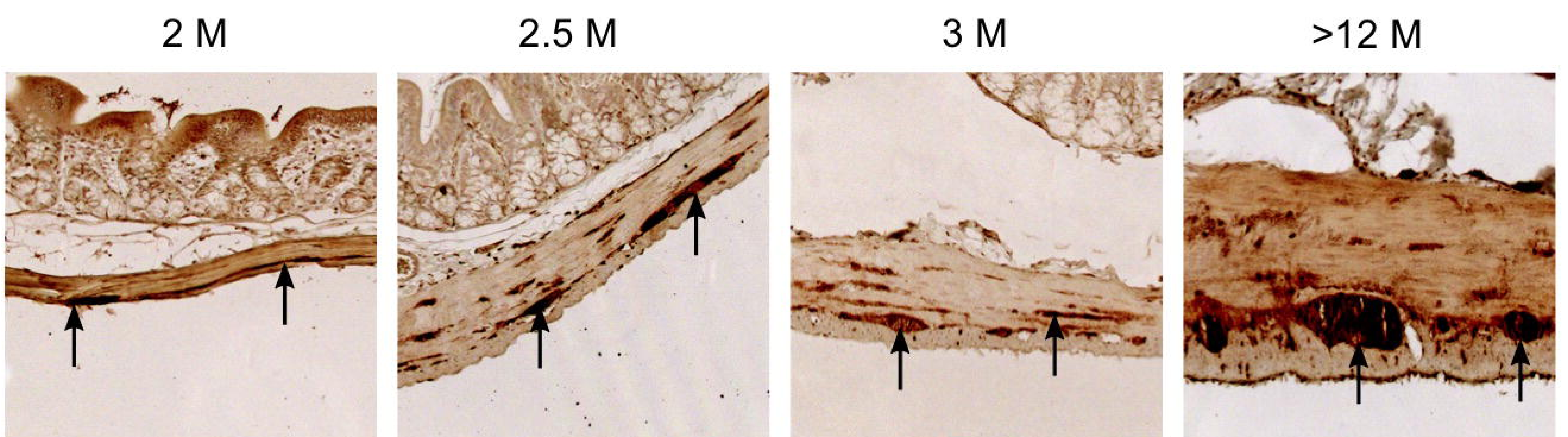

TG
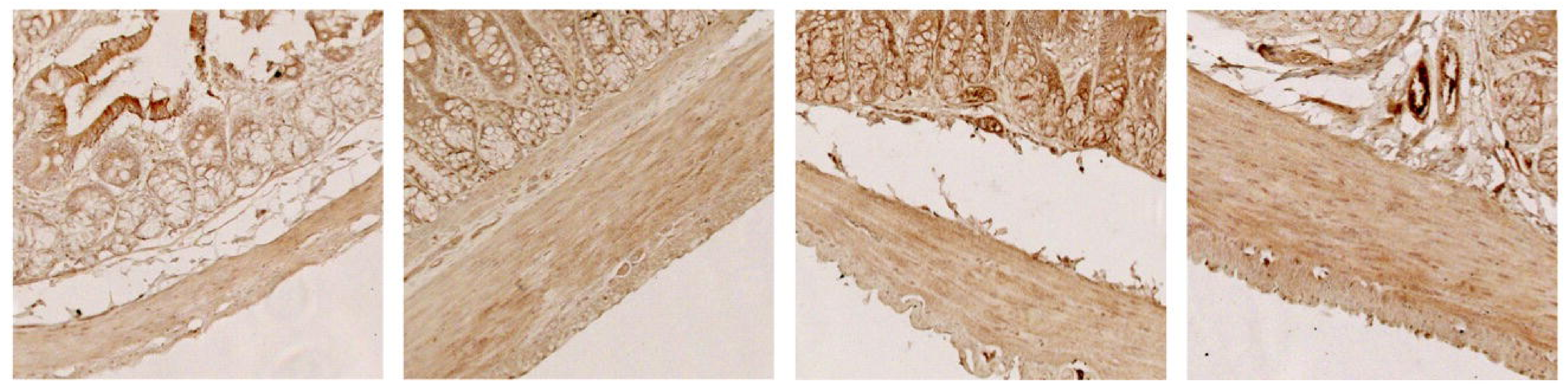

TG (- blocking peptide)

Human primary

a-syn (p129) antibody

(\#ab51253) EP1356Y

TG (+ blocking peptide)

C

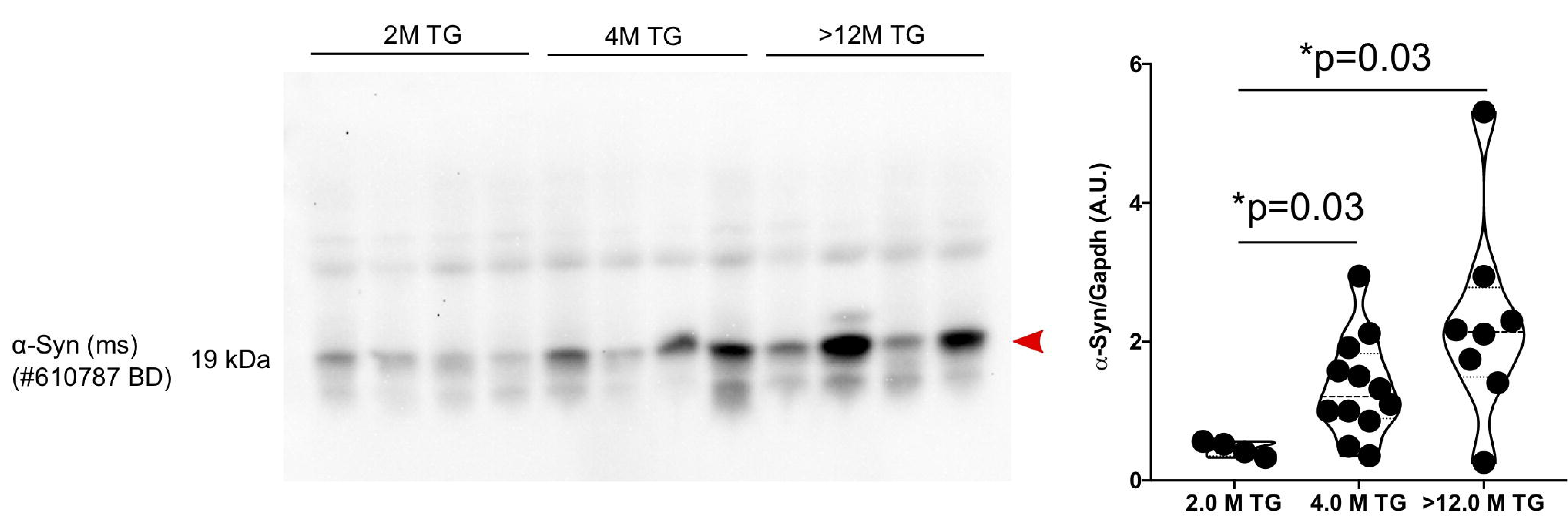

Fig. 4 
a

Cartoon showing the measurements of $\mathrm{Na}^{+}$currents in the rat colon epithelial tissues

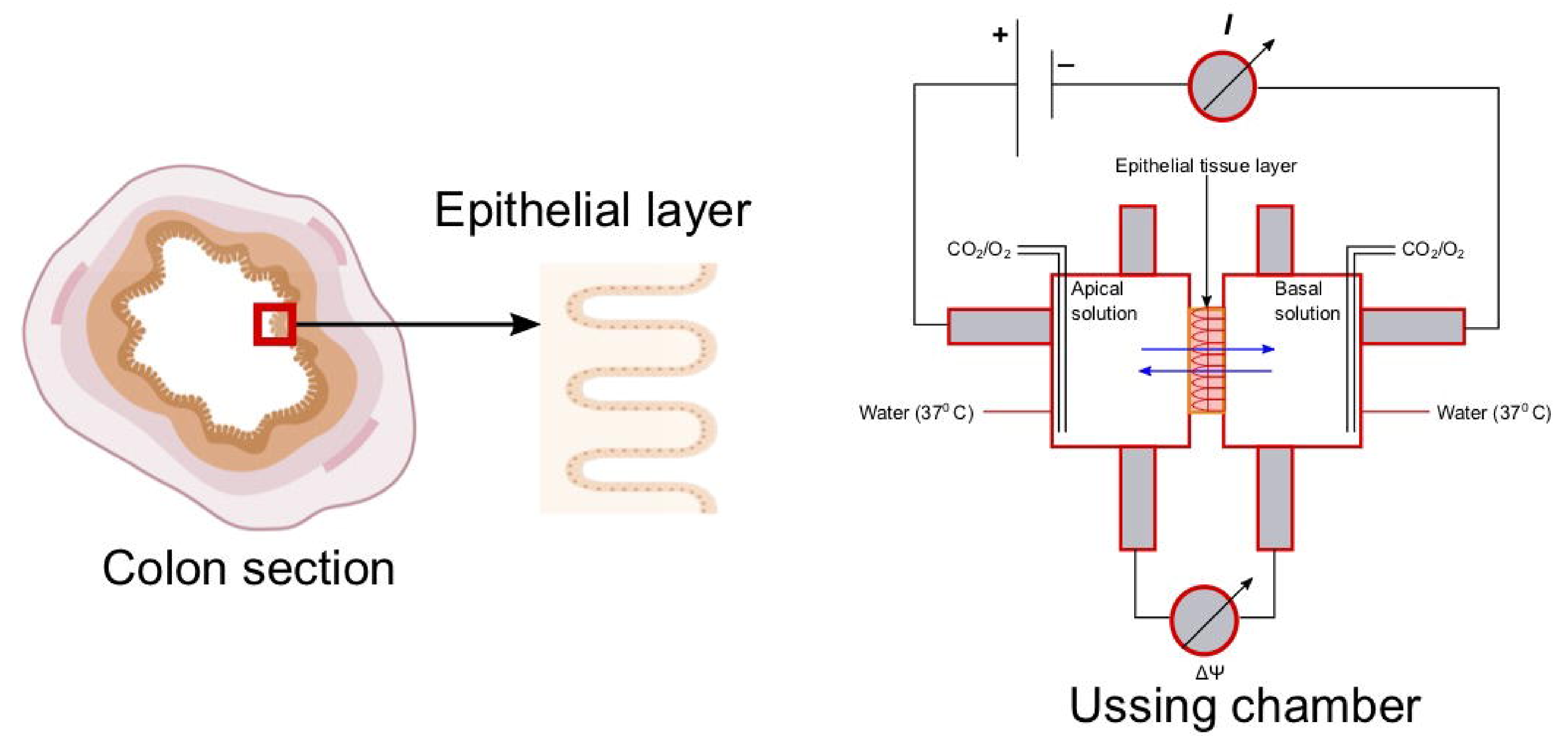

b

$2 M$

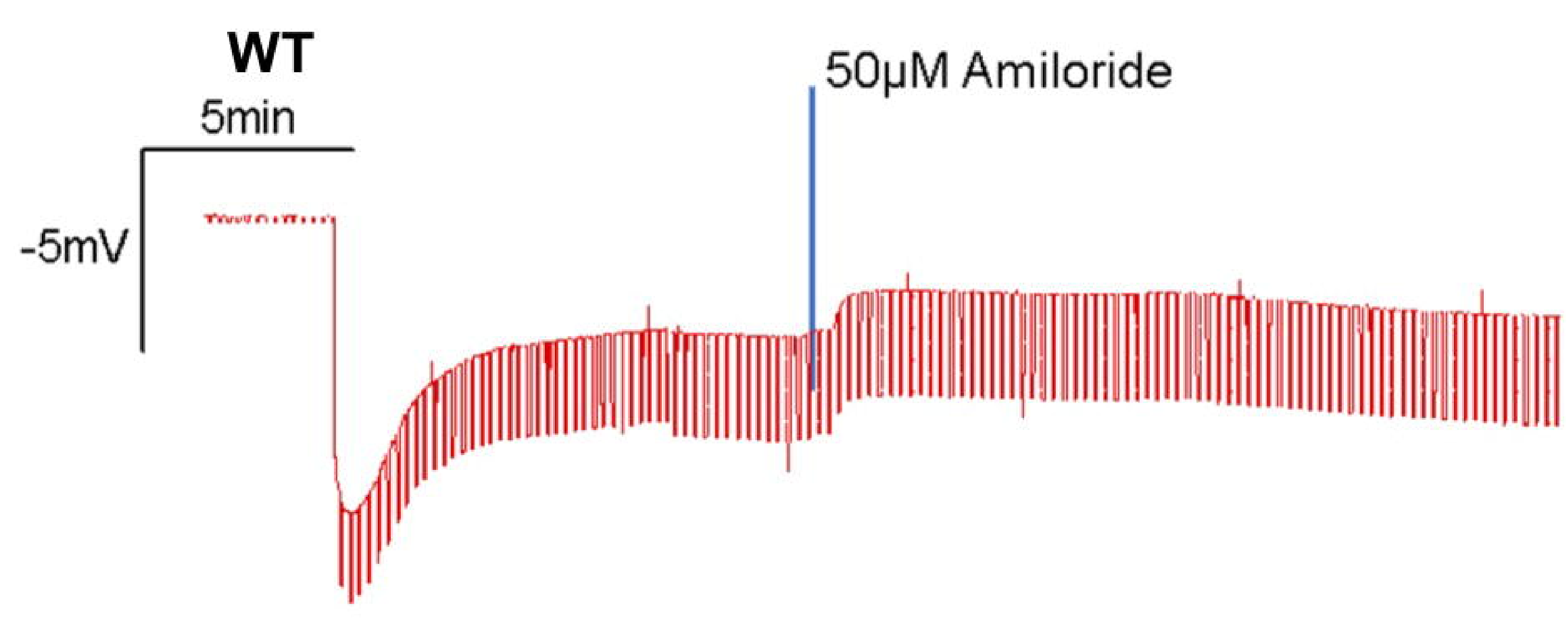

TG

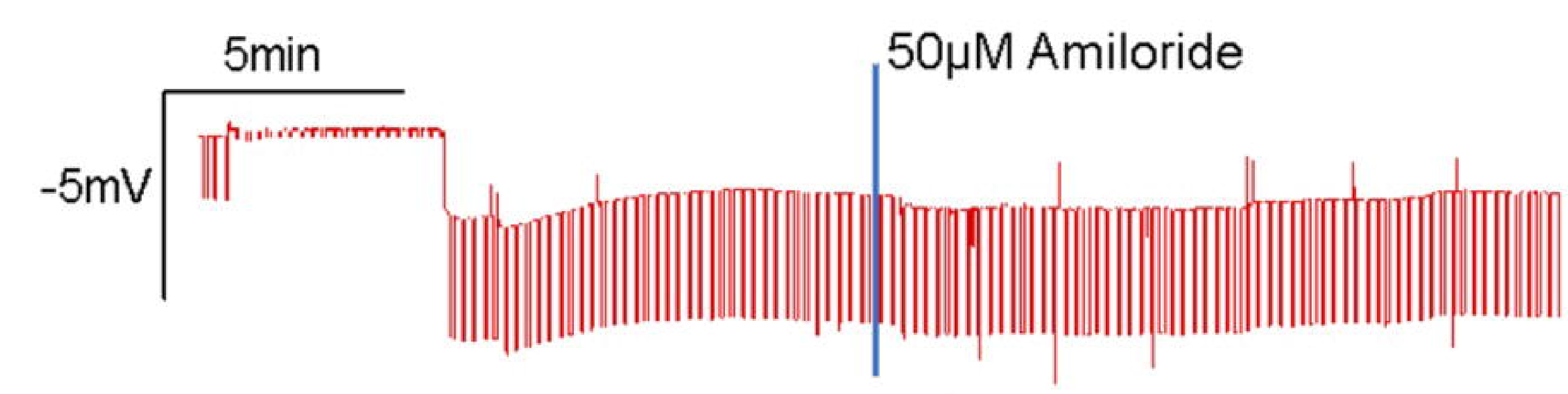

C

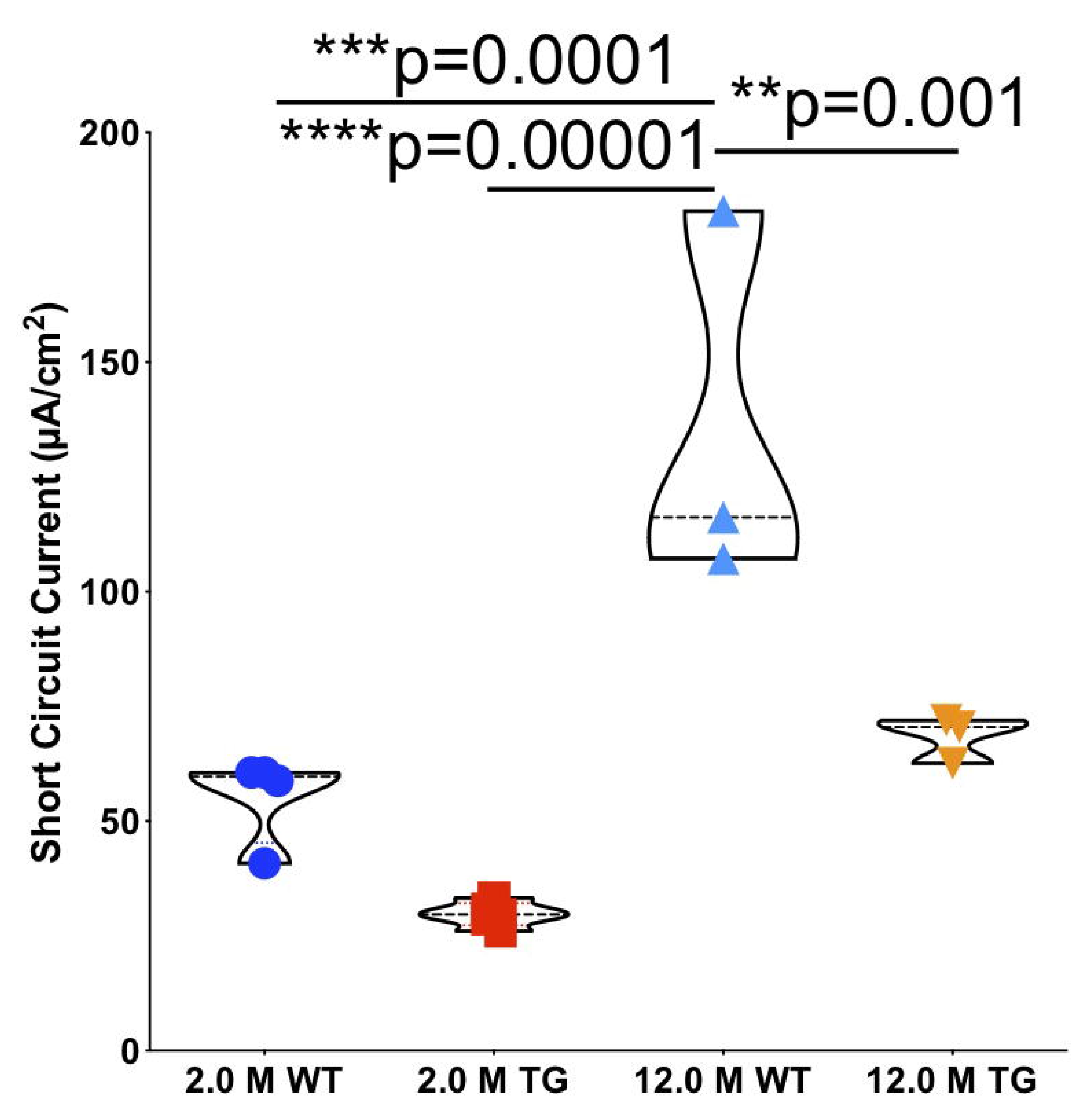

d

Hypoxanthine

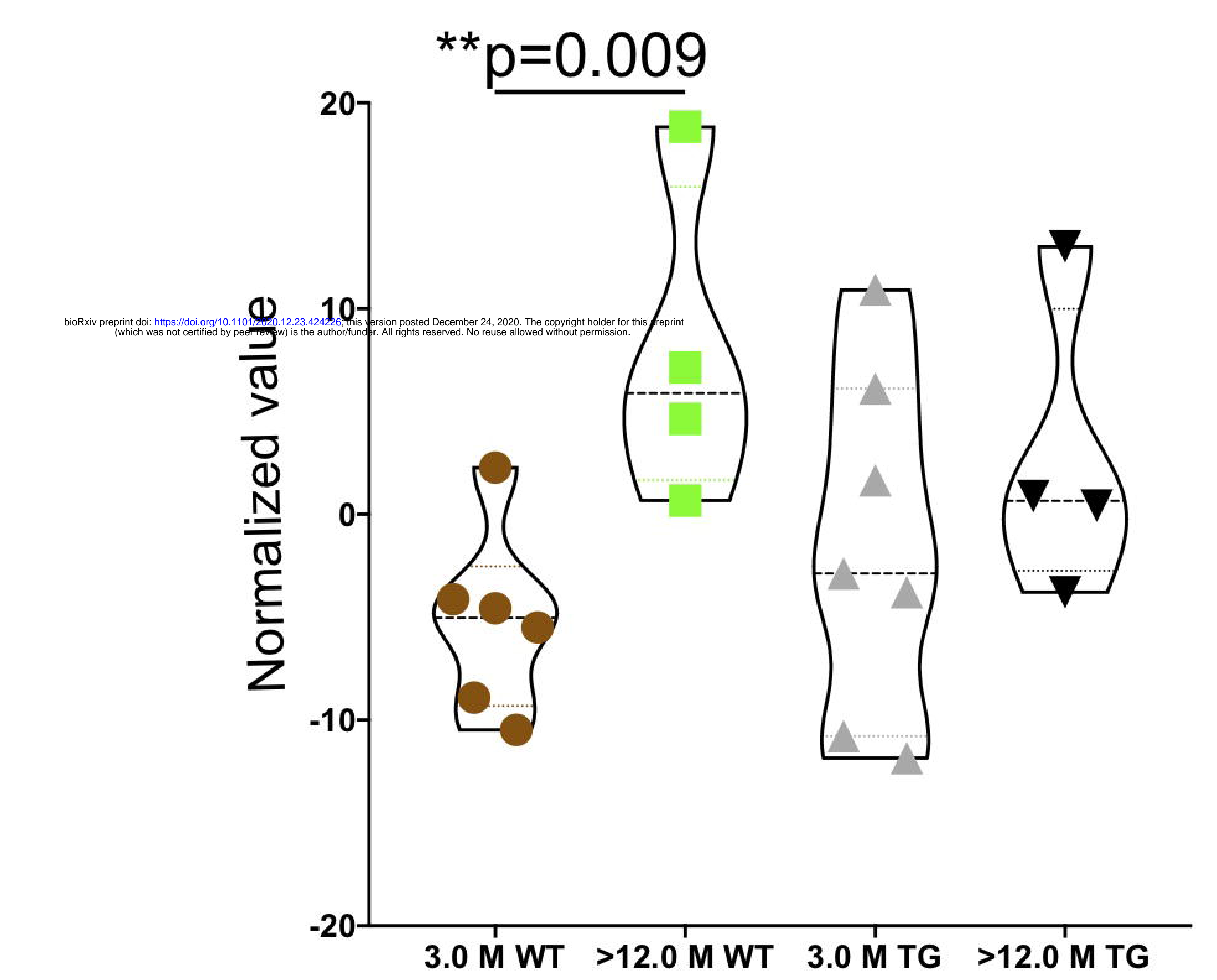

$12 \mathrm{M}$
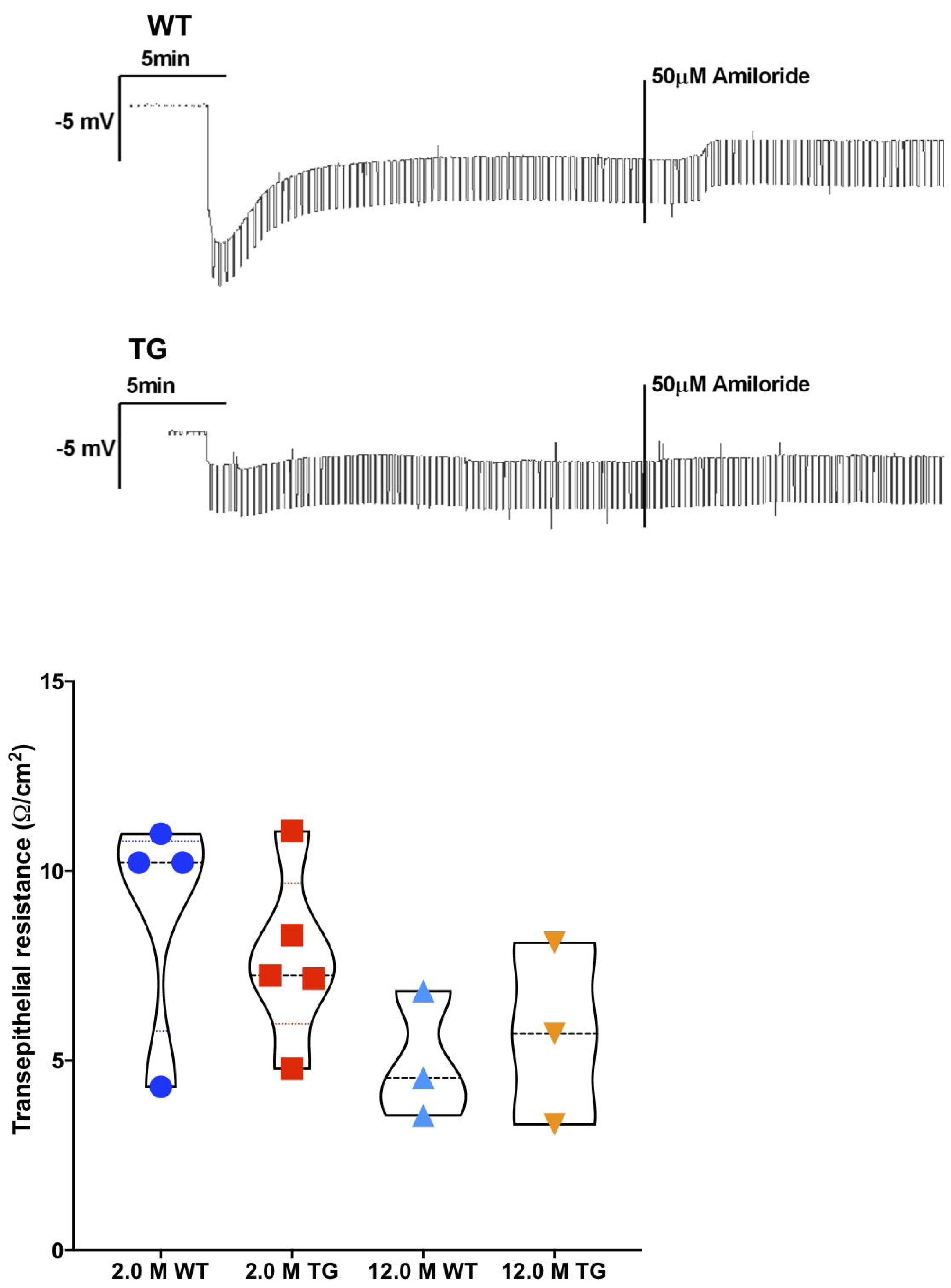

Fig. 5 


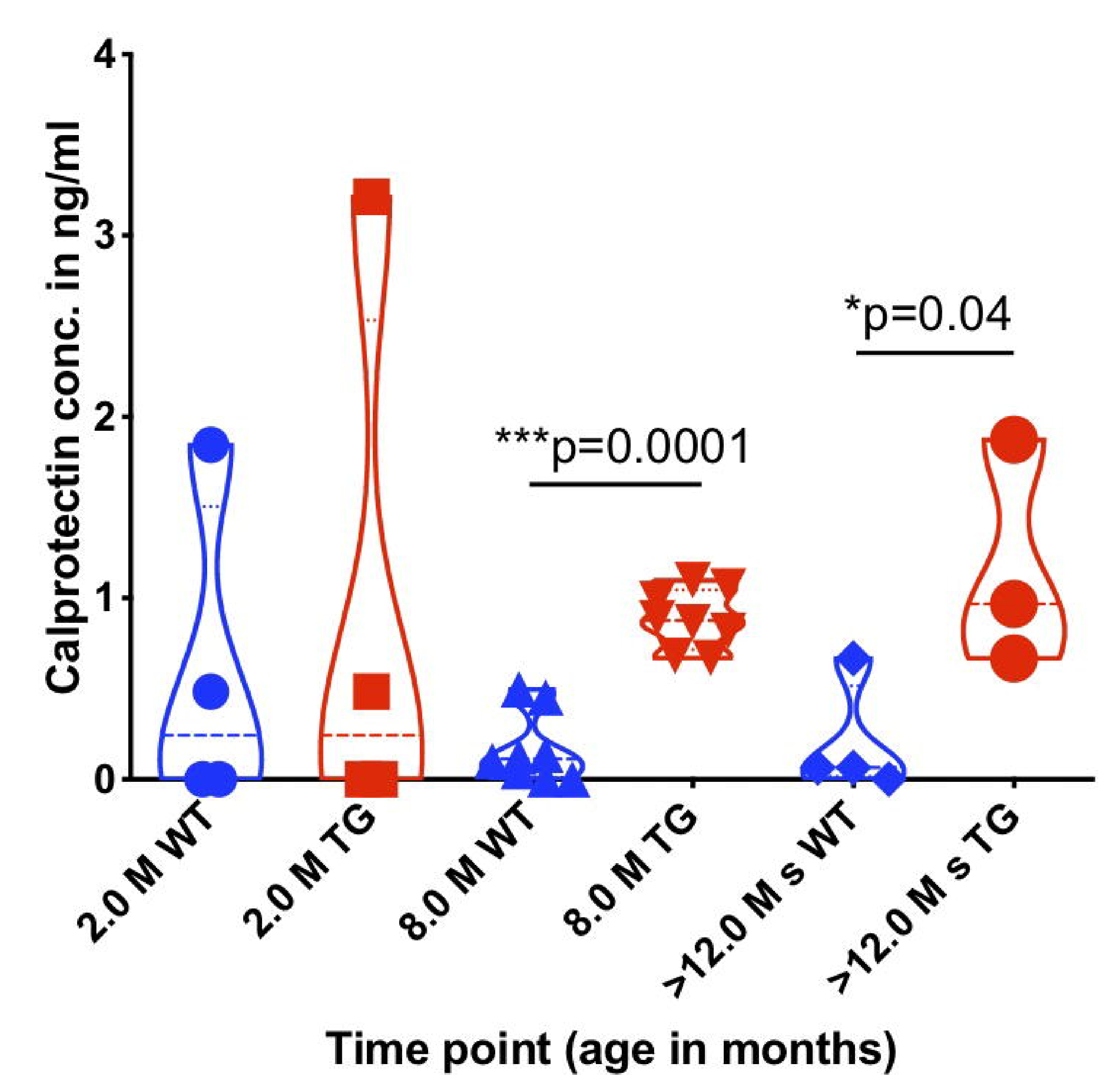

C

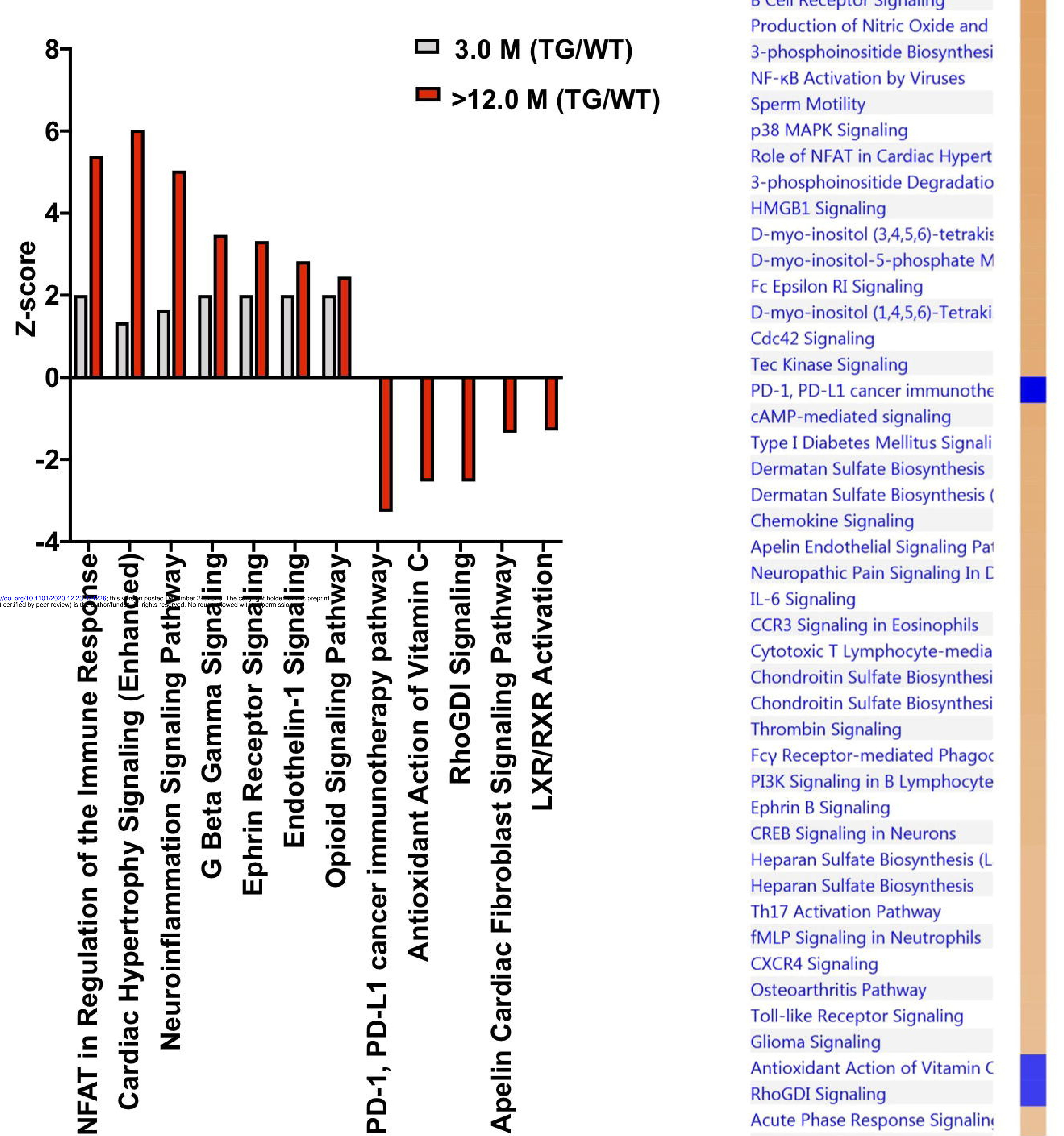

Canonical pathways 12 Neuroinflammation Signaling $\mathrm{P}$ $G$ Beta Gamma Signaling Systemic Lupus Erythematosus PKCӨ Signaling in T Lymphocyt: Phospholipase C Signaling Dendritic Cell Maturation COS-iCOSL Signaling in Opioid Signaling Pathway II-8 Signaling

Calcium-induced T Lymphocyte NF-kB Signaling

sation Signali Recognition Rer CD28 Signaling in T Helper Cell: Superpathway of Inositol Phosf

mily GTPase ell Receptor Signaling

Cardiac Hyper Role of NFAT in Regulation of $t$
Legend 1. $3.0 \mathrm{M}(\mathrm{TG} / \mathrm{WT})$ 2. $>12.0 \mathrm{M}(\mathrm{TG} / \mathrm{WT})$
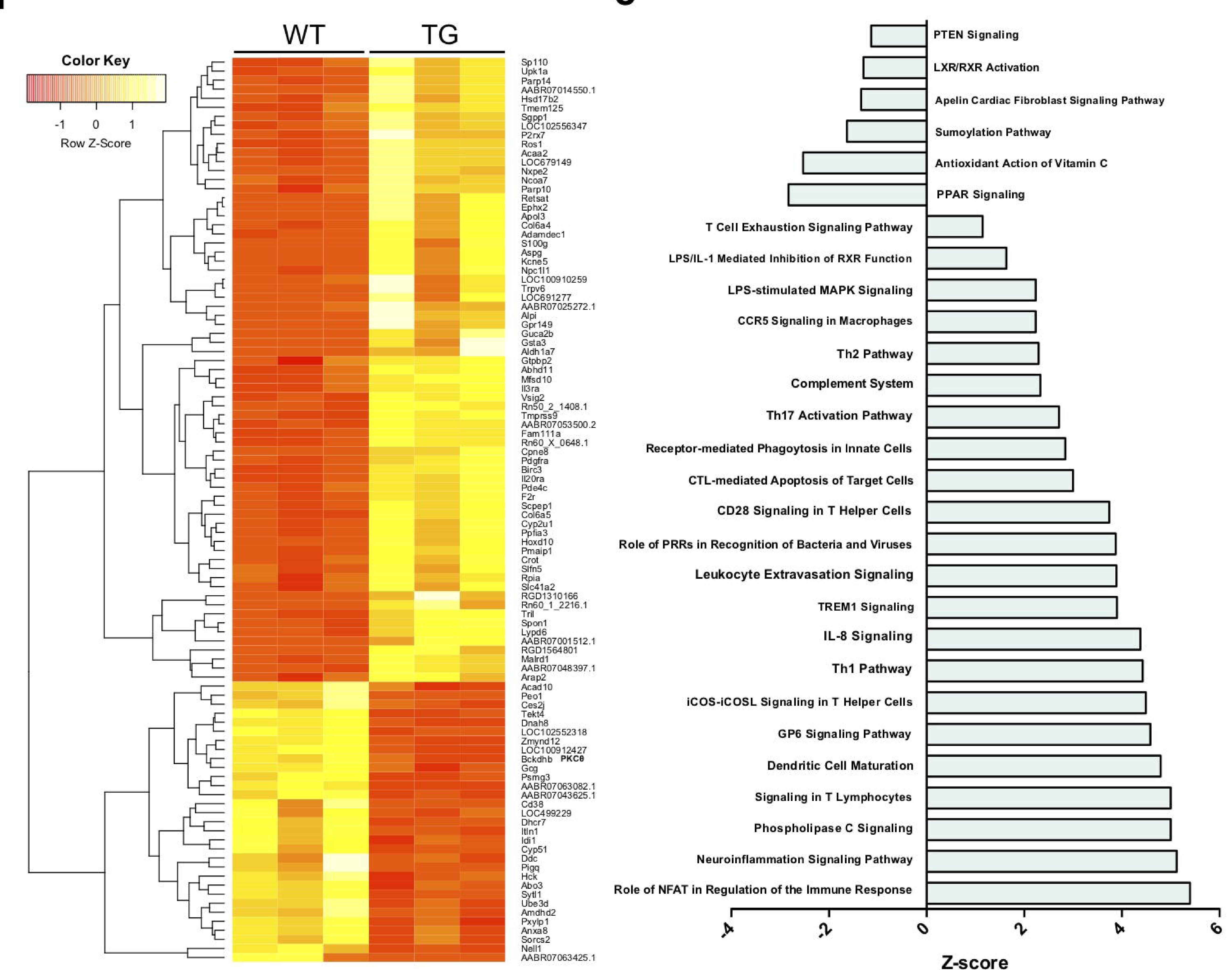

f
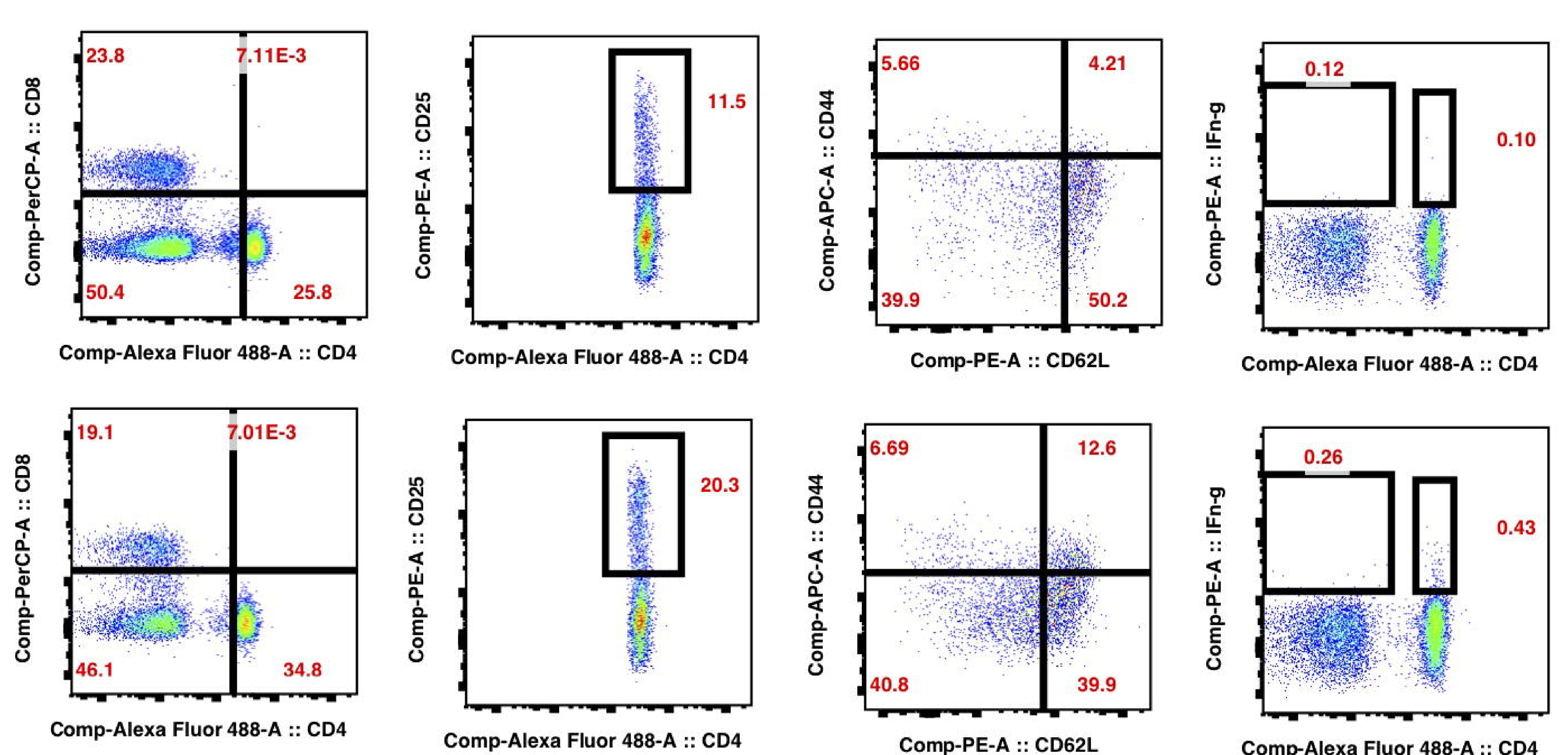

TG
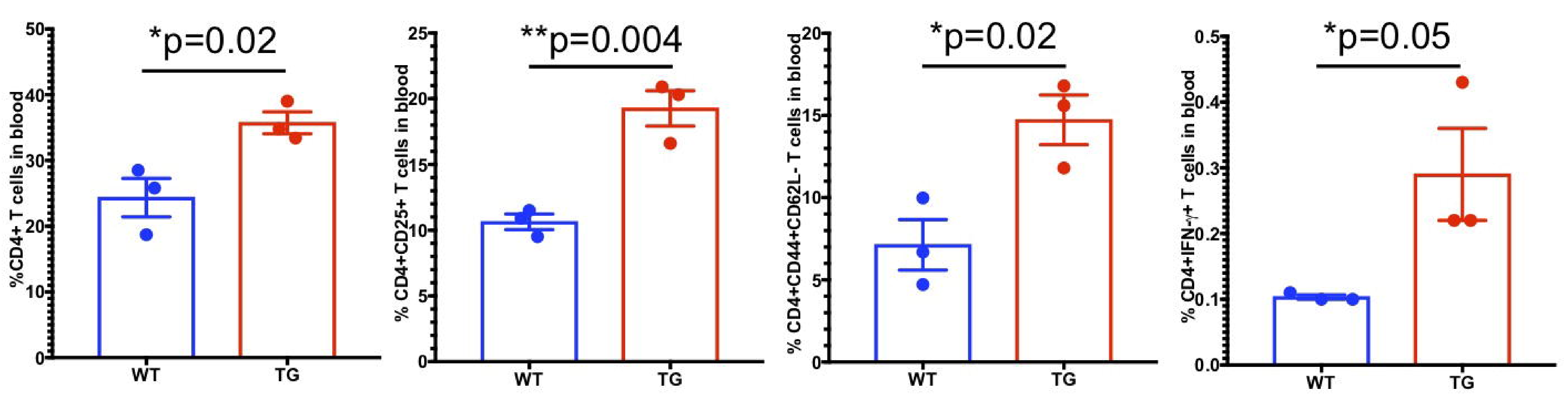


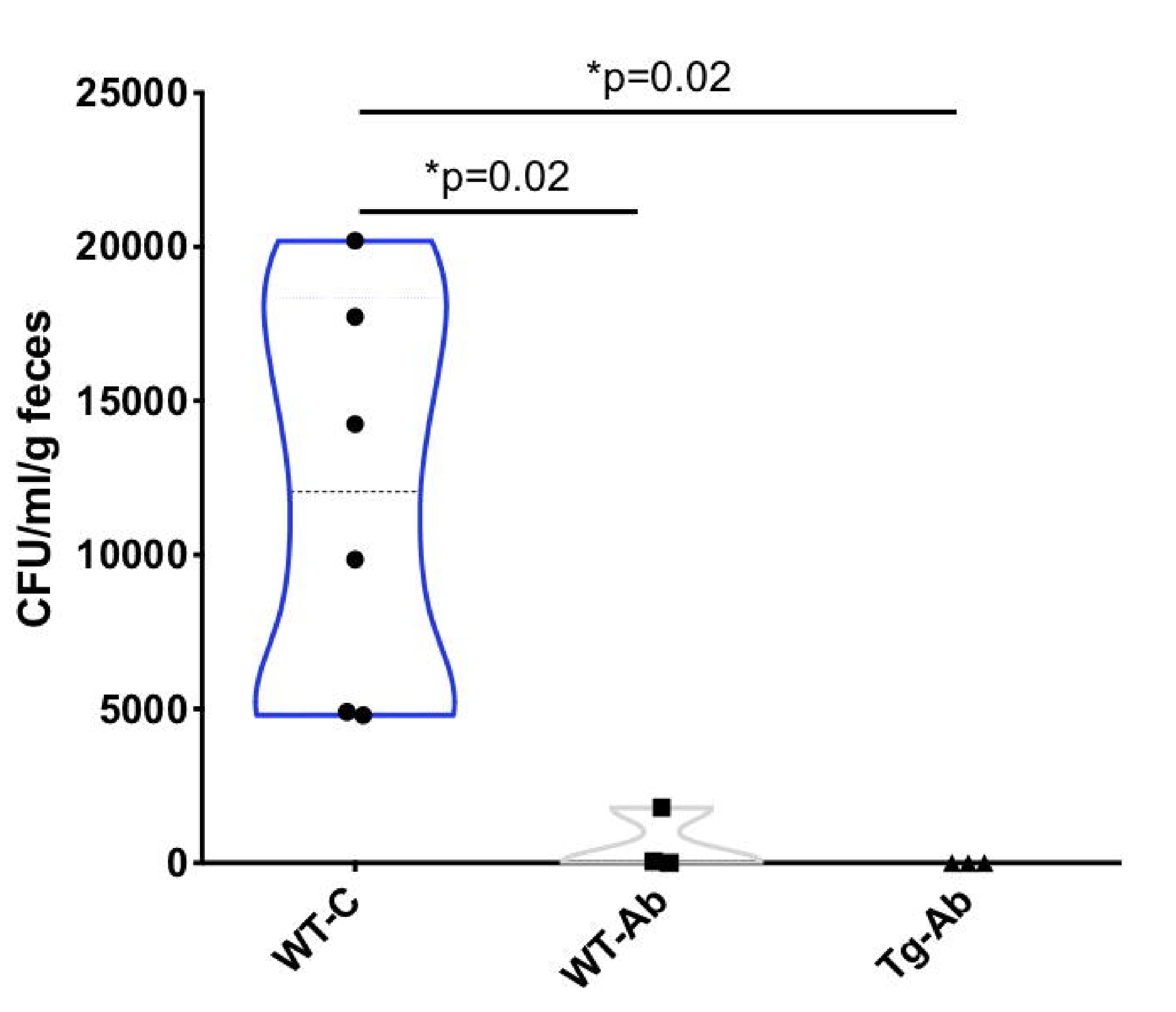

e

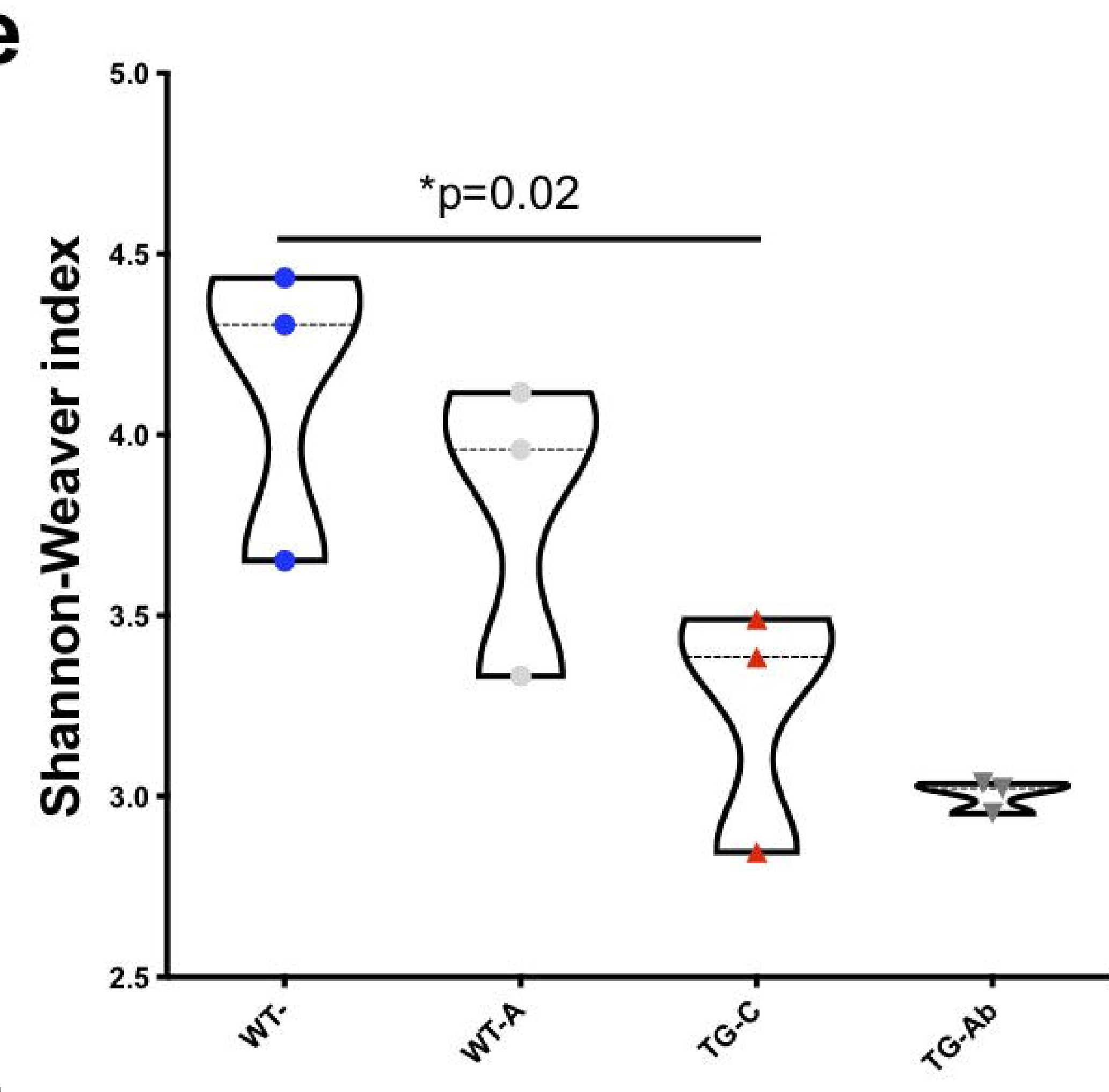

h
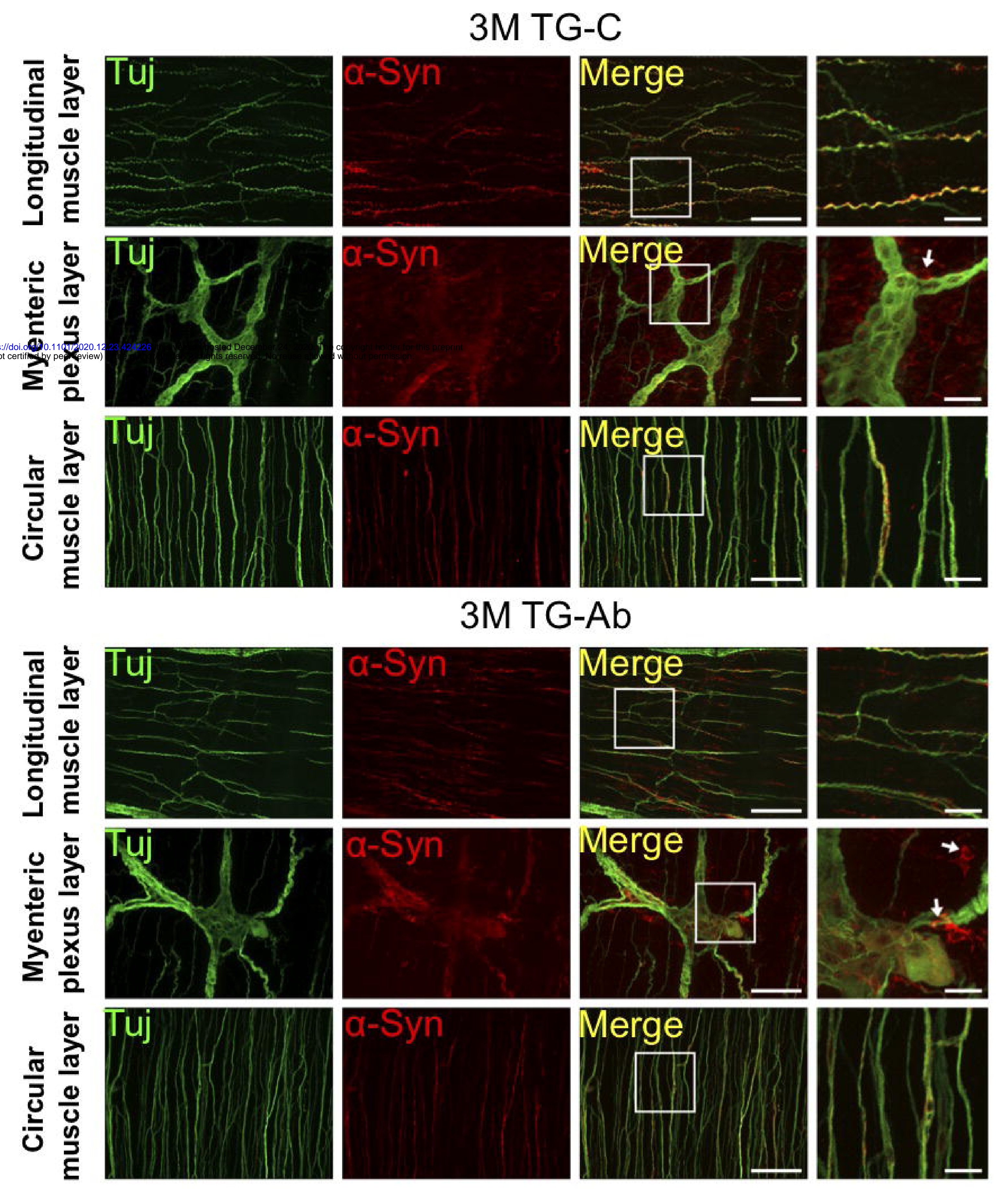

f
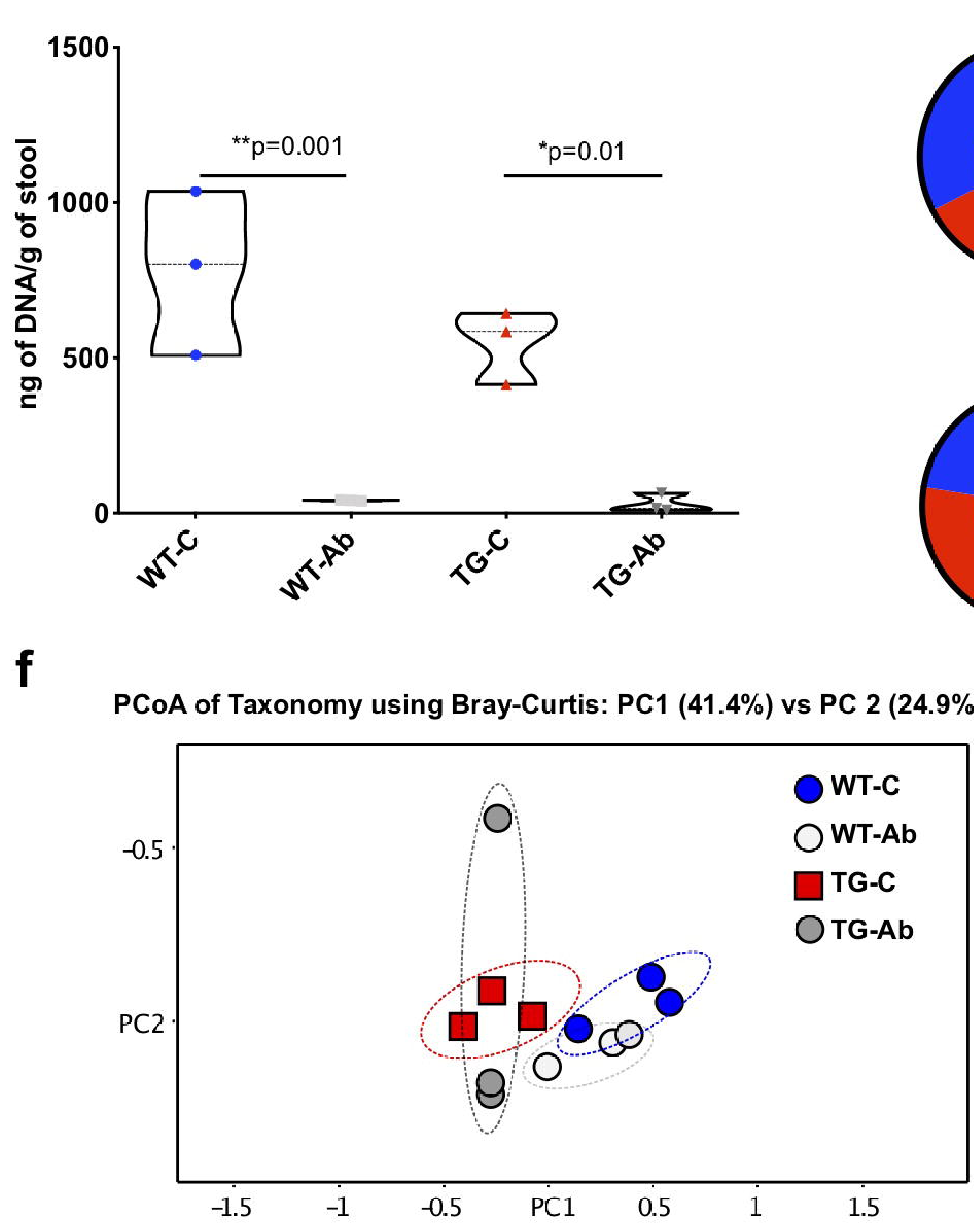

i

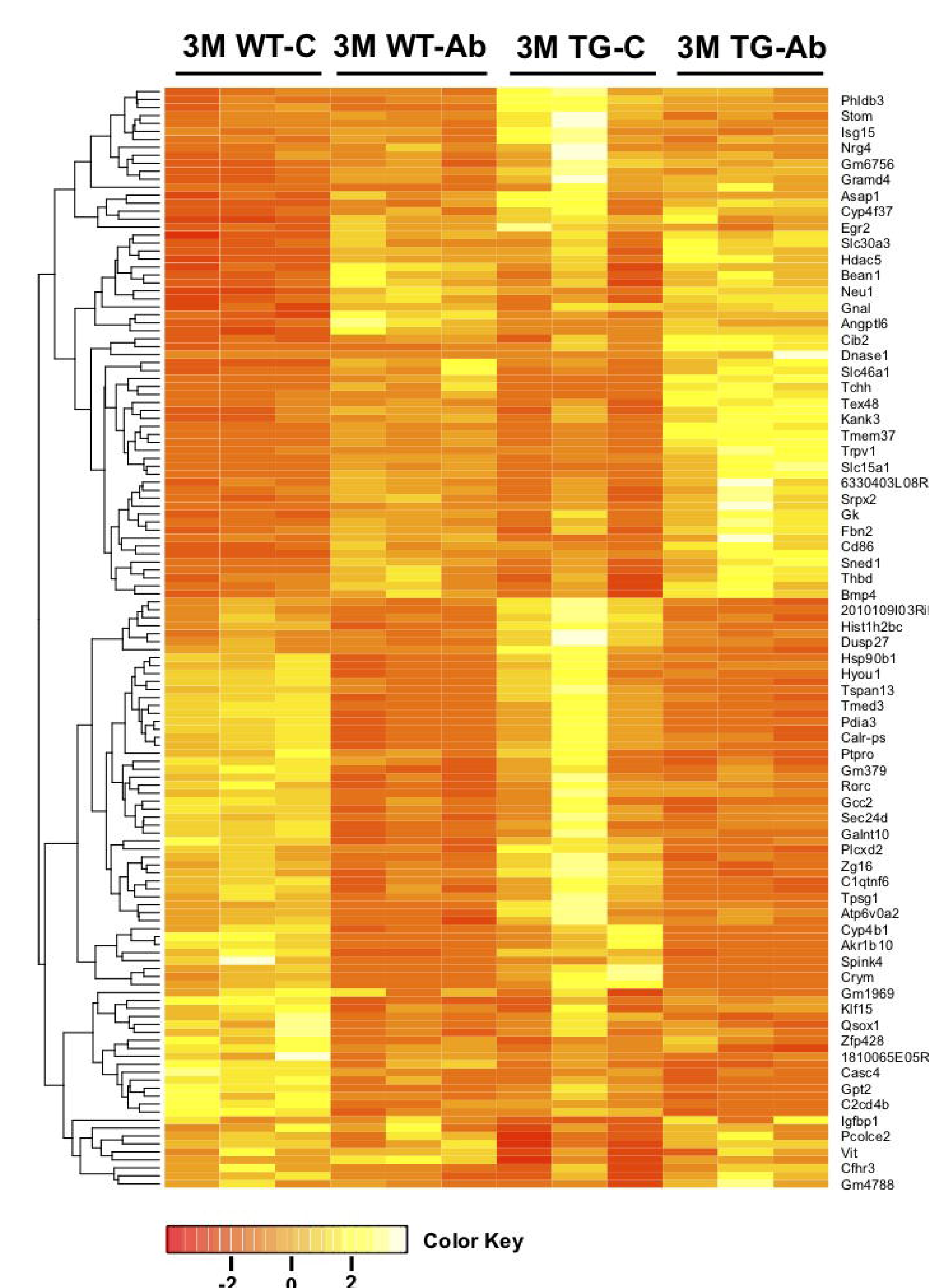

Control

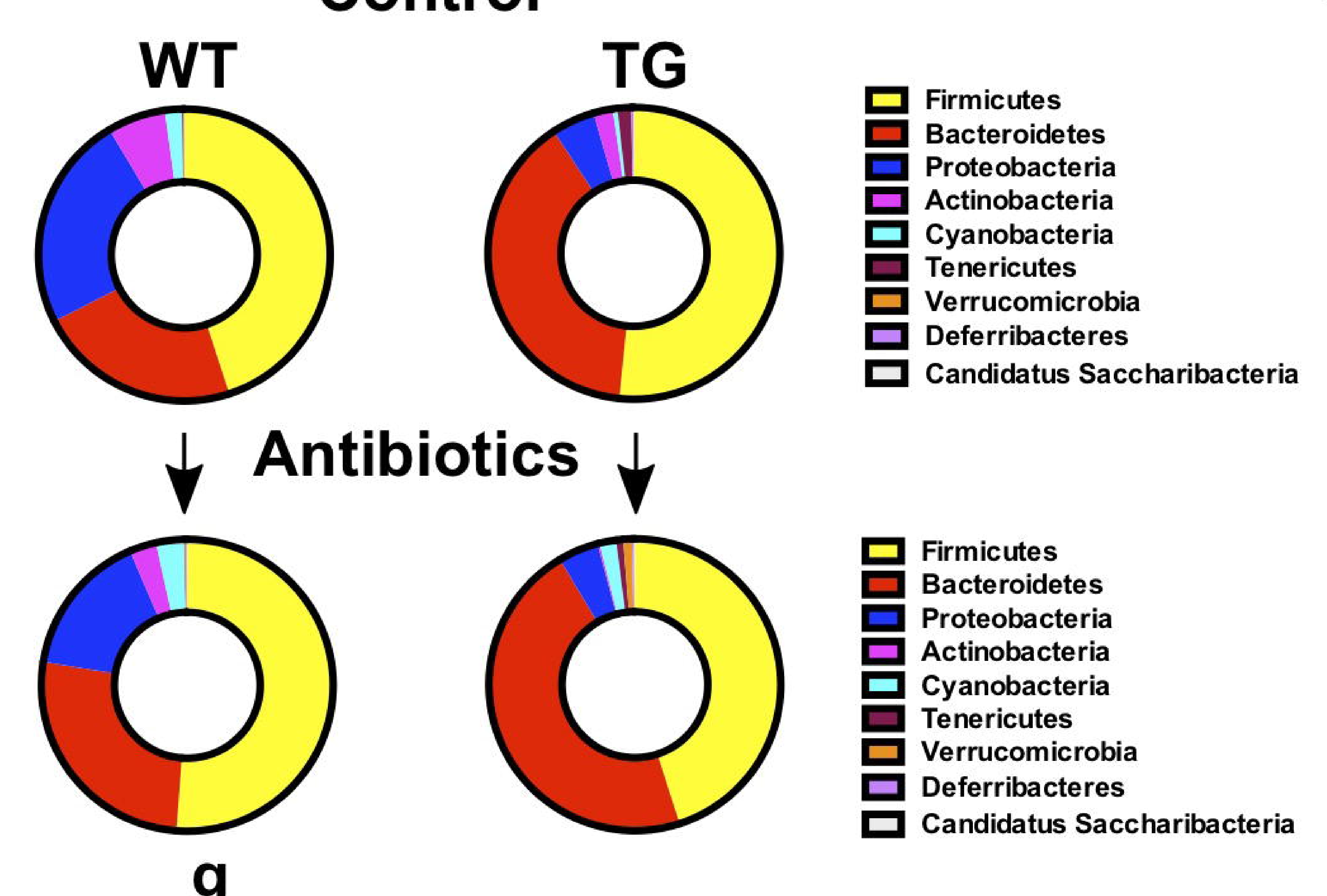

g

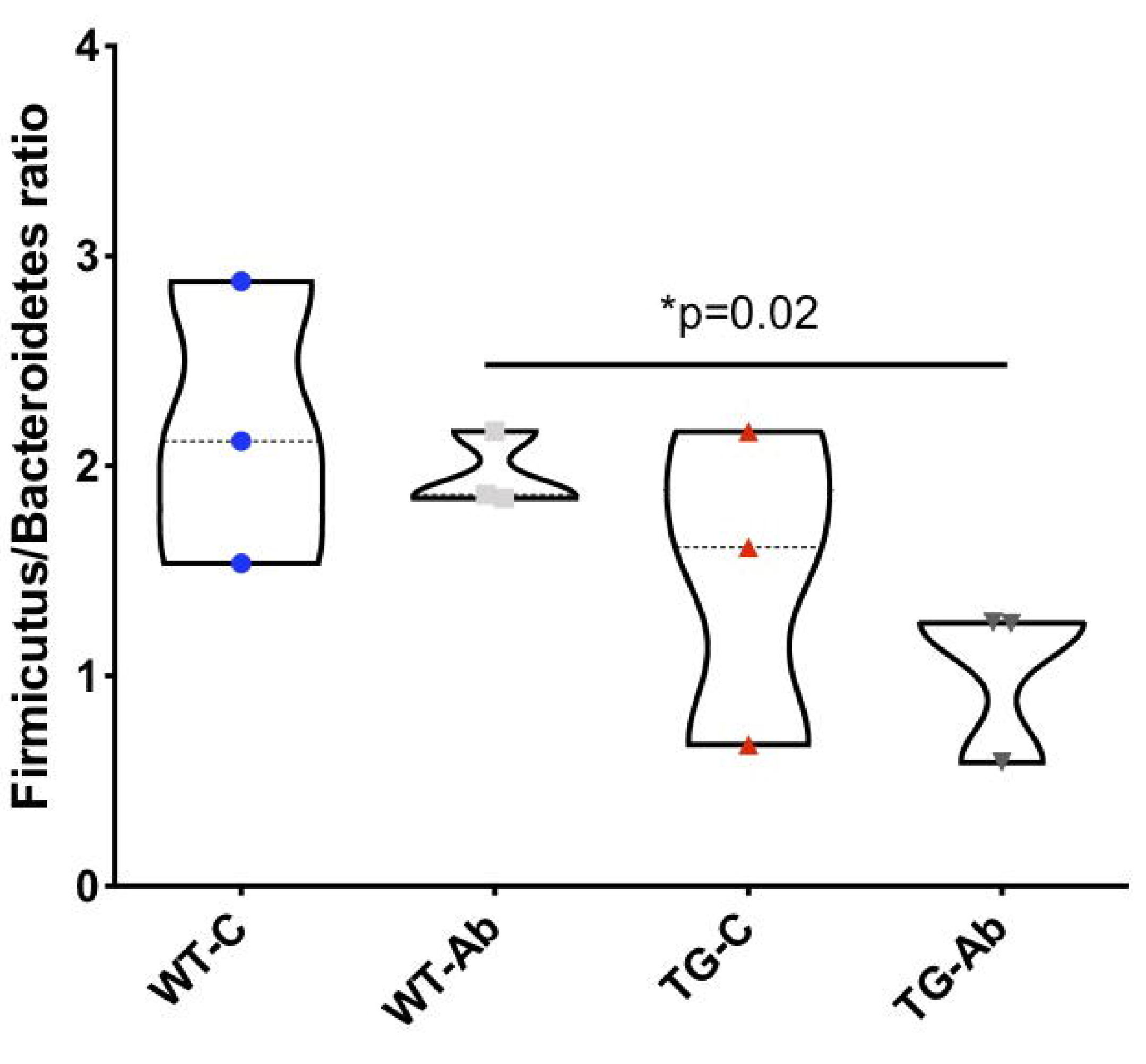

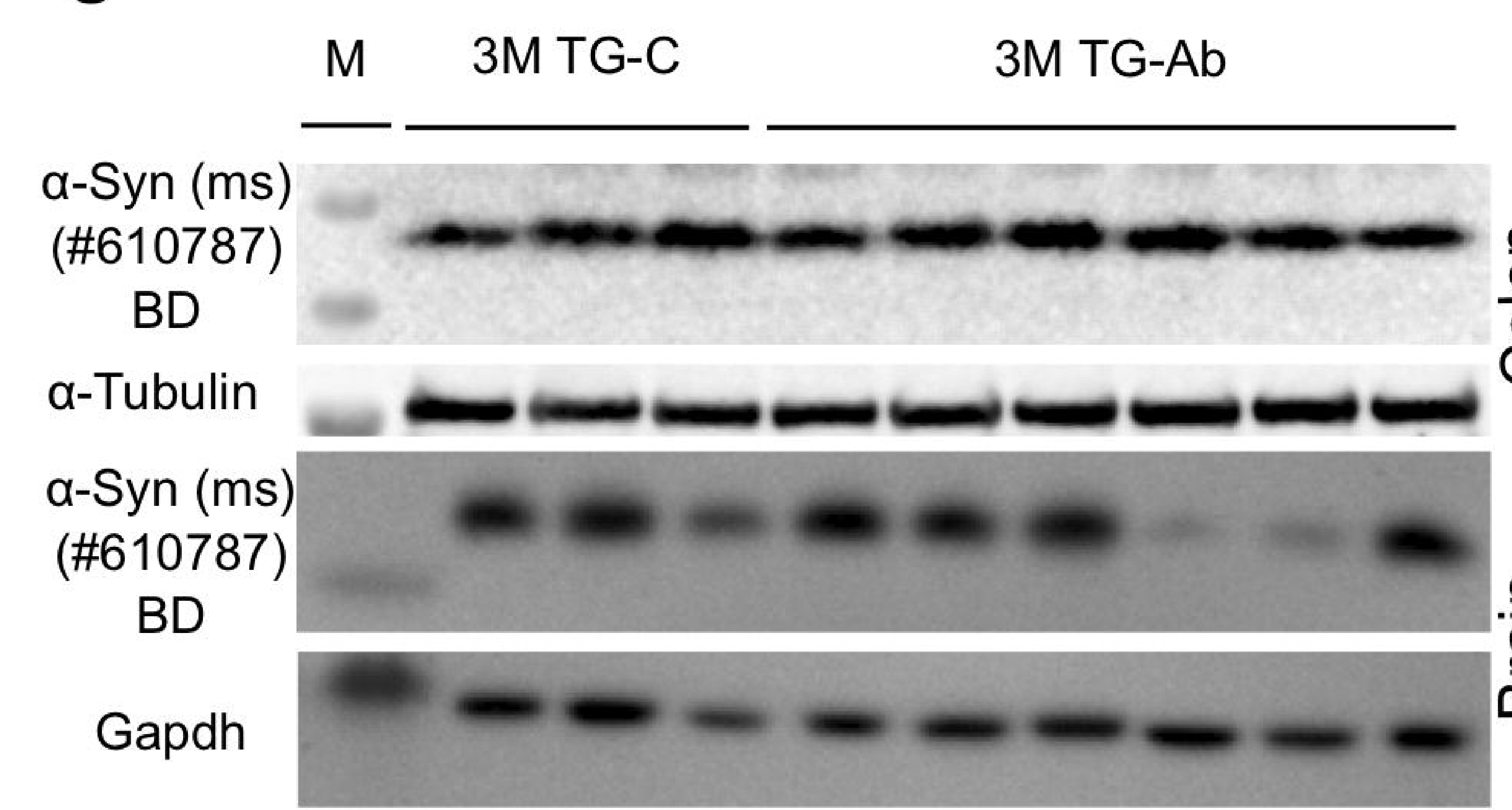

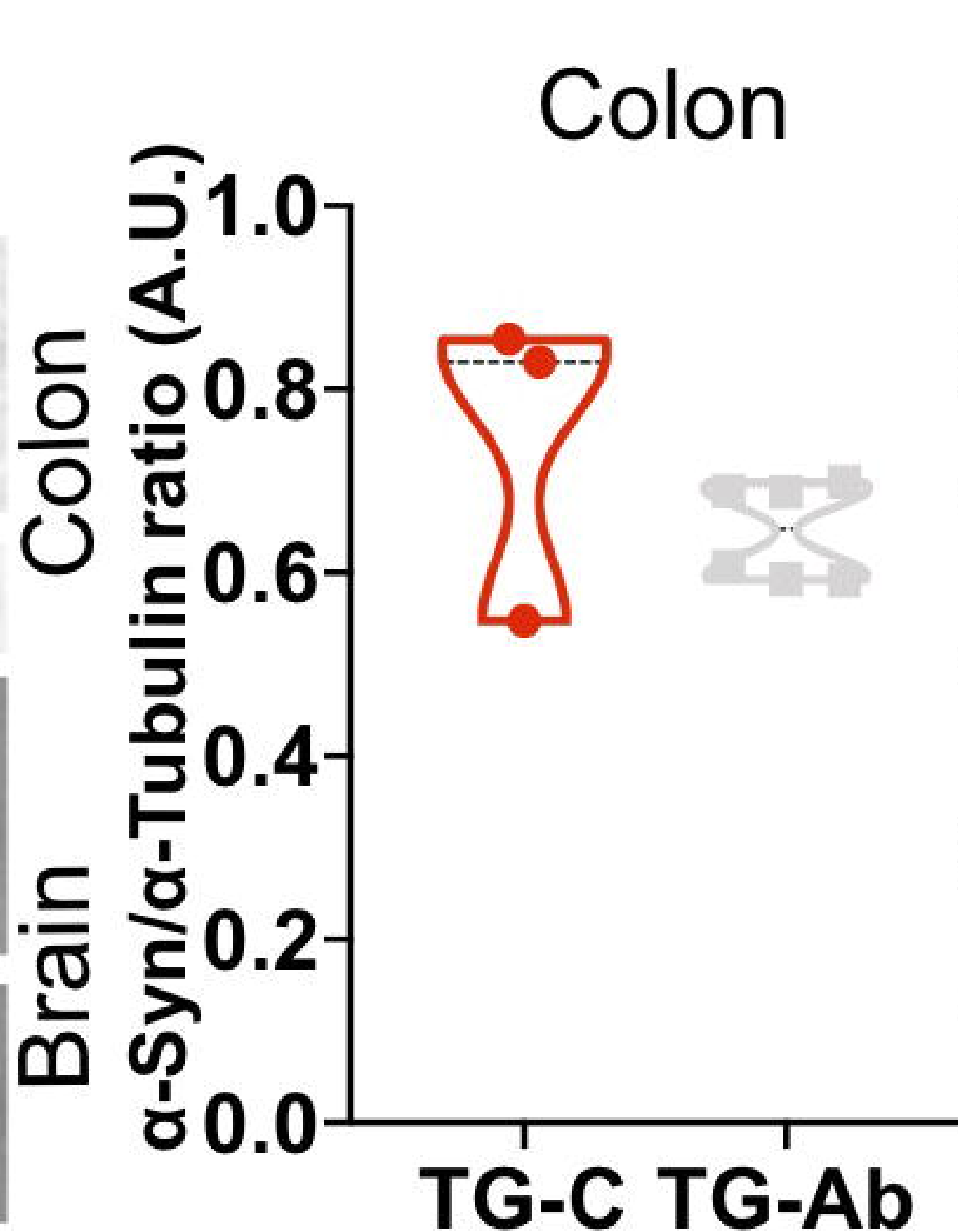

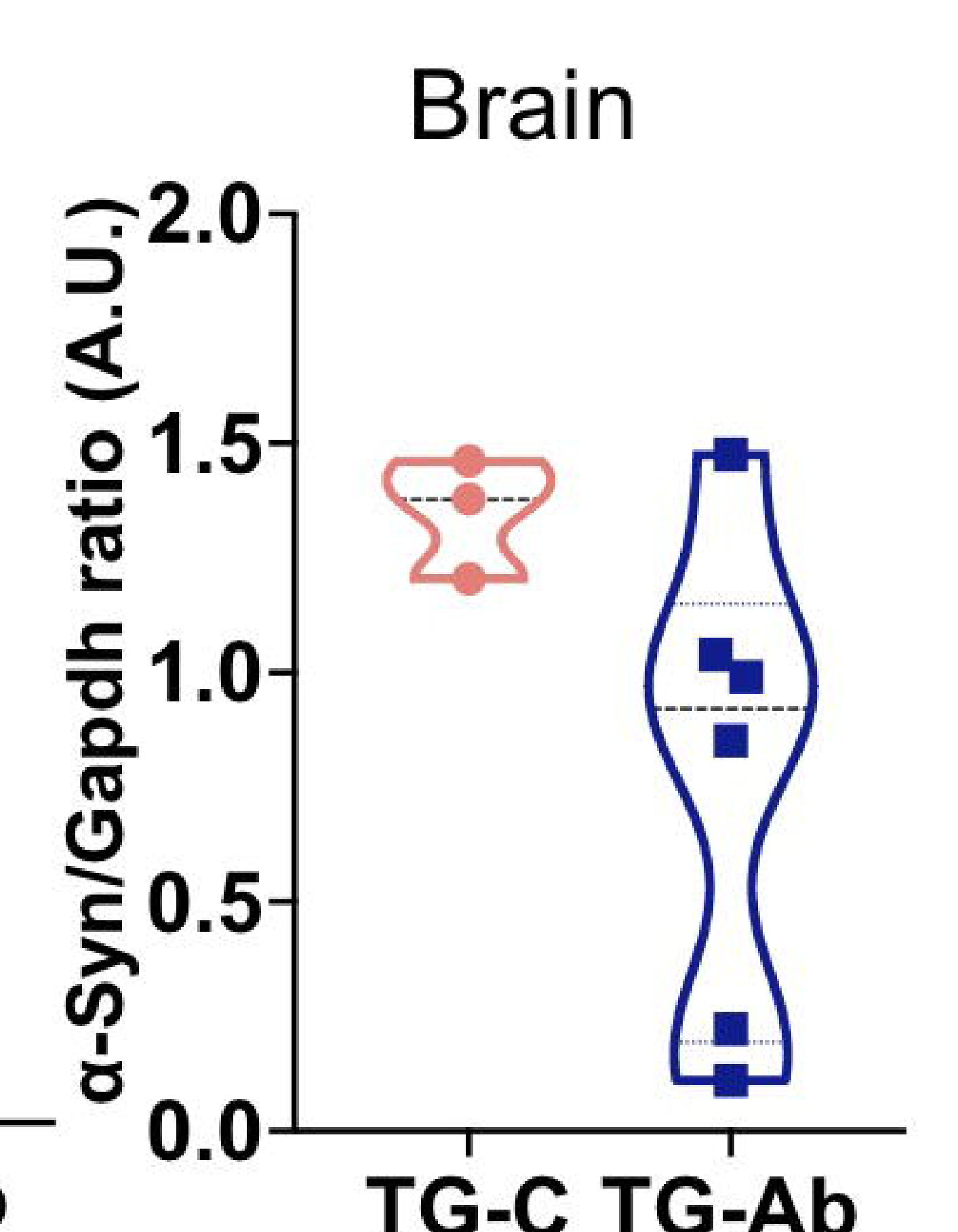

j
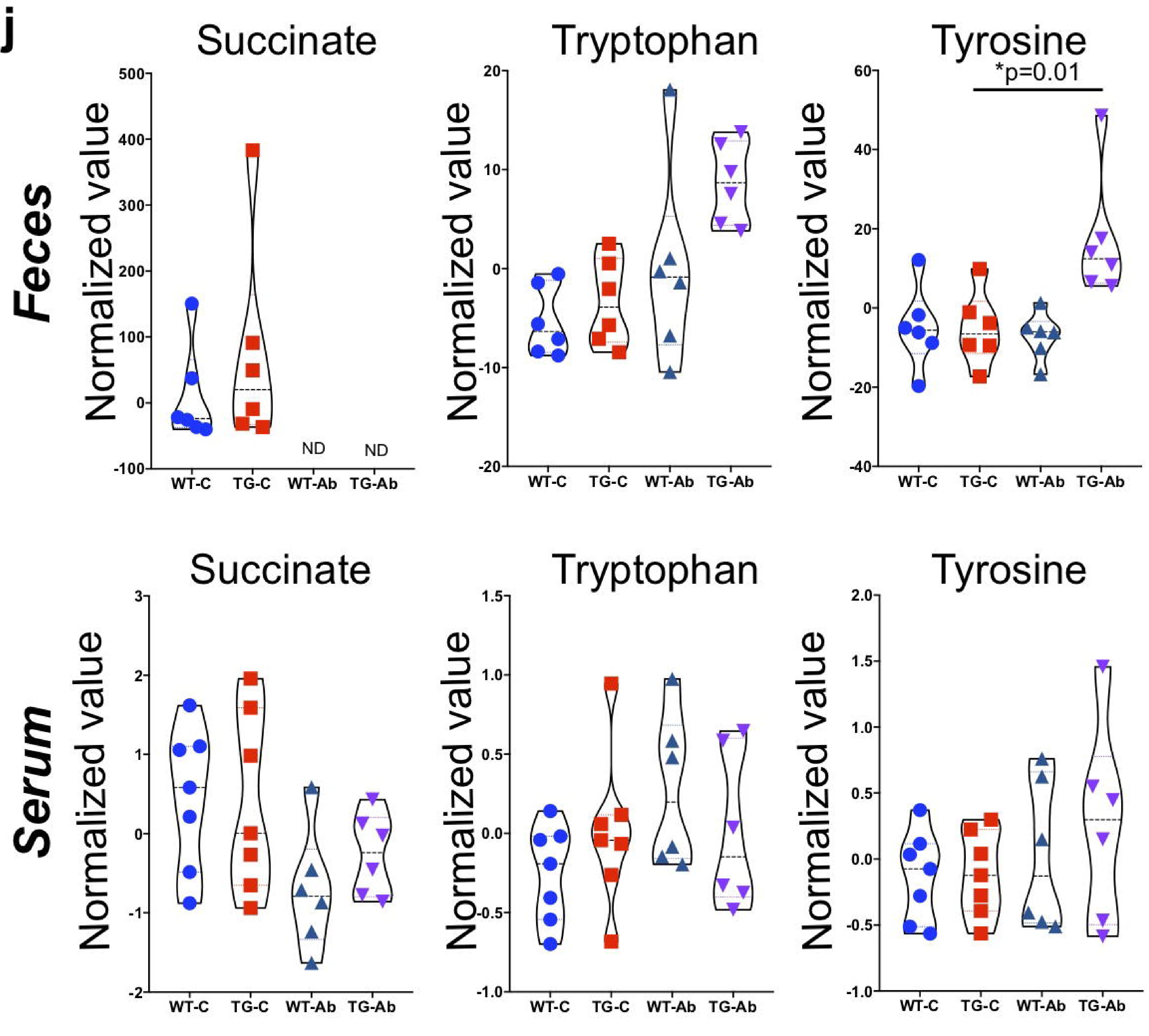

Fig. 7 